\title{
43. DEEP SEA DRILLING PROJECT DRILL SITES 530 AND 532 IN THE ANGOLA BASIN AND ON THE WALVIS RIDGE: INTERPRETATION OF INDUCTION LOG DATA, SONIC LOG DATA, AND LABORATORY SOUND VELOCITY, DENSITY, POROSITY-DERIVED REFLECTION COEFFICIENTS, AND VANE SHEAR STRENGTH ${ }^{1}$
}

\author{
Robert E. Boyce, Deep Sea Drilling Project, Scripps Institution of Oceanography, La Jolla, California
}

\begin{abstract}
From 0 to $277 \mathrm{~m}$ at Site 530 are found Holocene to Miocene diatom ooze, nannofossil ooze, marl, clay, and debrisflow deposits; from 277 to $467 \mathrm{~m}$ are Miocene to Oligocene mud; from 467 to $1103 \mathrm{~m}$ are Eocene to late Albian Cenomanian interbedded mudstone, marlstone, chalk, clastic limestone, sandstone, and black shale in the lower portion; from 1103 to $1121 \mathrm{~m}$ are basalts.

In the interval from 0 to $467 \mathrm{~m}$, in Holocene to Oligocene pelagic oozes, marl, clay, debris flows, and mud, velocities are 1.5 to $1.8 \mathrm{~km} / \mathrm{s}$; below $200 \mathrm{~m}$ velocities increase irregularly with increasing depth. From 0 to $100 \mathrm{~m}$, in Holocene to Pleistocene diatom and nannofossil oozes (excluding debris flows), velocities are approximately equivalent to that of the interstitial seawater, and thus acoustic reflections in the upper $100 \mathrm{~m}$ are primarily caused by variations in density and porosity. Below 100 or $200 \mathrm{~m}$, acoustic reflections are caused by variations in both velocity and density. From 100 to $467 \mathrm{~m}$, in Miocene-Oligocene nannofossil ooze, clay, marl, debris flows, and mud, acoustic anisotropy irregularly increases to $10 \%$, with 2 to $5 \%$ being typical.

From 467 to $1103 \mathrm{~m}$ in Paleocene to late Albian Cenomanian interbedded mudstone, marlstone, chalk, clastic limestone, and black shale in the lower portion of the hole, velocities range from 1.6 to $5.48 \mathrm{~km} / \mathrm{s}$, and acoustic anisotropies are as great as $47 \%(1.0 \mathrm{~km} / \mathrm{s})$ faster horizontally. Mudstone and uncemented sandstone have anisotropies which irregularly increase with increasing depth from 5 to $10 \%(0.2 \mathrm{~km} / \mathrm{s})$. Calcareous mudstones have the greatest anisotropies, typically $35 \%(0.6 \mathrm{~km} / \mathrm{s})$.

Below $1103 \mathrm{~m}$, basalt velocities ranged from 4.68 to $4.98 \mathrm{~km} / \mathrm{s}$. A typical value is about $4.8 \mathrm{~km} / \mathrm{s}$.

In situ velocities are calculated from velocity data obtained in the laboratory. These are corrected for in situ temperature, hydrostatic pressure, and porosity rebound (expansion when the overburden pressure is released). These corrections do not include rigidity variations caused by overburden pressures. These corrections affect semiconsolidated sedimentary rocks the most (up to $0.25 \mathrm{~km} / \mathrm{s}$ faster). These laboratory velocities appear to be greater than the velocities from the sonic log.

Reflection coefficients derived from the laboratory data, in general, agree with the major features on the seismic profiles. These indicate more potential reflectors than indicated from the reflection coefficients derived using the Gearhart-Owen Sonic Log from 625 to $940 \mathrm{~m}$, because the Sonic Log data average thin beds.

Porosity-density data versus depth for mud, mudstone, and pelagic oozes agree with data for similar sediments as summarized in Hamilton (1976). At depths of about $400 \mathrm{~m}$ and about $850 \mathrm{~m}$ are zones of relatively higher porosity mudstones, which may suggest anomalously high pore pressure; however, they are more probably caused by variations in grain-size distribution and lithology.

Electrical resistivity (horizontal) from 625 to $950 \mathrm{~m}$ ranged from about 1.0 to $4.0 \mathrm{ohm}-\mathrm{m}$, in Maestrichtian to Santonian-Coniacian mudstone, marlstone, chalk, clastic limestone, and sandstone. An interstitial-water resistivity curve did not indicate any unexpected lithology or unusual fluid or gas in the pores of the rock. These logs were above the black shale beds.

From 0 to $100 \mathrm{~m}$ at Sites 530 and 532, the vane shear strength on undisturbed samples of Holocene-Pleistocene diatom and nannofossil ooze uniformly increases from about $80 \mathrm{~g} / \mathrm{cm}^{2}$ to about $800 \mathrm{~g} / \mathrm{cm}^{2}$. From 100 to $300 \mathrm{~m}$, vane shear strength of Pleistocene-Miocene nannofossil ooze, clay, and marl are irregular versus depth with a range of 500 to 2300 $\mathrm{g} / \mathrm{cm}^{2}$; and at Site 532 the vane shear strength appears to decrease irregularly and slightly with increasing depth (gassy zone). Vane shear strength values of gassy samples may not be valid, for the samples may be disturbed as gas evolves, and the sediments may not be gassy at in situ depths.
\end{abstract}

\section{INTRODUCTION}

This chapter reports certain relationships among physical properties, using samples from Deep Sea Drilling Project Sites 530 and 532 and selected well logs obtained at Hole 530A. Site 530 is in the Angola Basin and Site 532 is on the Walvis Ridge. These features are in the southeastern Atlantic Ocean (Fig. 1). The principal aims of this chapter are as follows:

\footnotetext{
${ }^{1}$ Hay, W. W., Sibuet, J.-C., et al., Init. Repts. DSDP, 75: Washington (U.S. Govt. Printing Office).
}

1) To introduce additional systematic studies of compressional-wave (sound) velocity and acoustic anisotropy for sediment, sedimentary rock, and basalt, and to determine their relationships to wet-bulk density and porosity. Acoustic impedance and reflection coefficients are derived. All of these are important for the proper interpretation of gravity, seismic reflection, seismic refraction, and sonobuoy data. Particularly important are data for very young sediments from the upper 100 or $200 \mathrm{~m}$ of the hole, which in the past have been too disturbed for proper study.

2) To study the Velocity and Induction Logs. This is important because if the porosity values derived from 


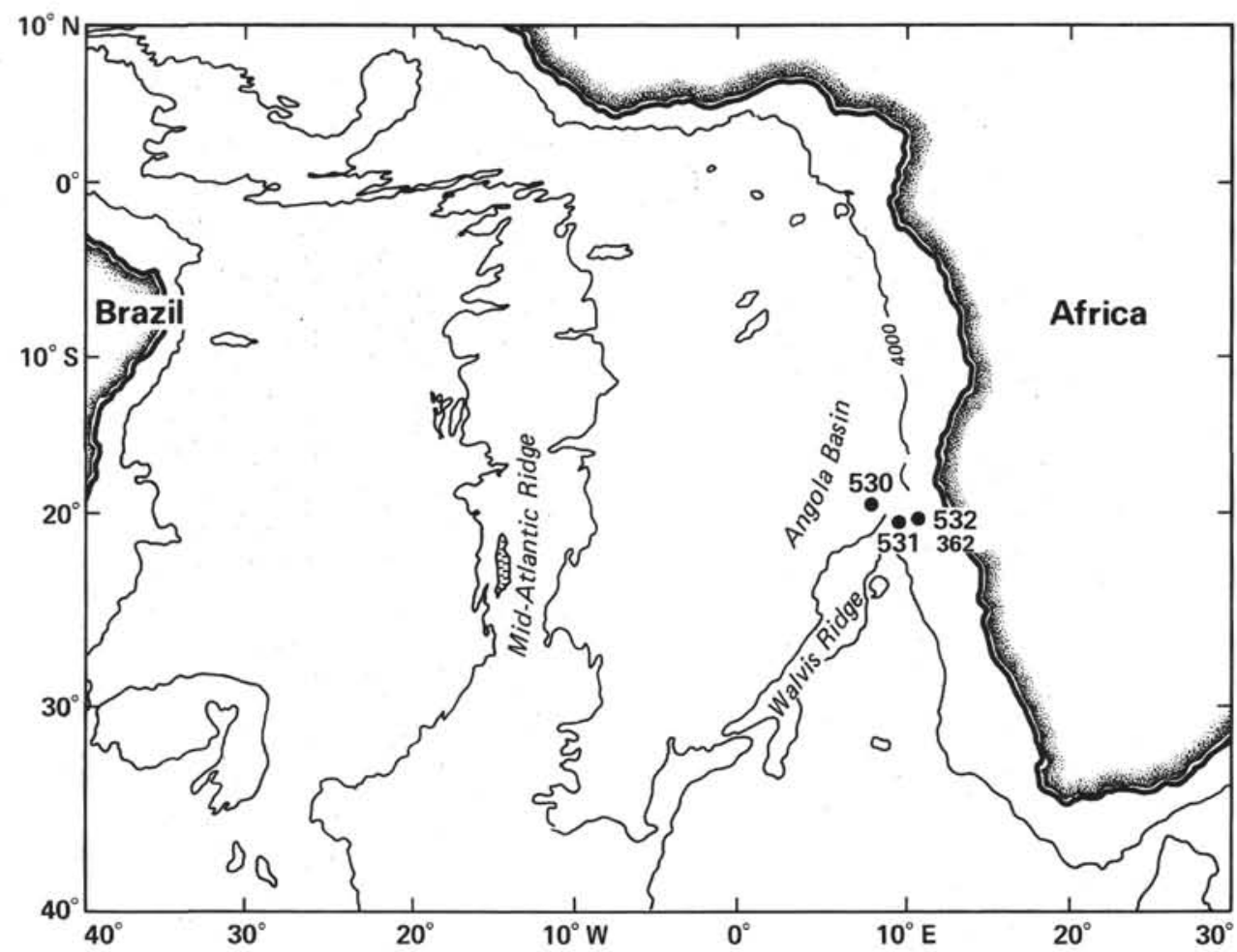

Figure 1. Location of Site 530 in the Angola Basin and Site 532 on the Walvis Ridge in the southeastern Atlantic Ocean off Africa. (Bathymetric contours $=4000 \mathrm{~m}$ depth.)

these logs do not agree within certain limits of error, then, assuming the logging data are accurate, one or more of the following is indicated: (a) conductive metallic minerals, (b) anomalies in the salinities of interstitial water, (c) an anomalous temperature, or (d) the presence of hydrocarbons.

3) To study vane shear strength on undisturbed samples of very young sediments from 0 to $300 \mathrm{~m}$ below the seafloor (these data are rare) as well as relationships to lithology, porosity, wet-bulk density, and compressional velocity.

\section{DEFINITIONS AND PROCEDURES}

Sediment and basalt classification is discussed in the Explanatory Notes to this volume. Wet-bulk density is the ratio of weight of the wet-saturated sediment or rock sample to its volume, expressed in $\mathrm{g} / \mathrm{cm}^{3}$. Wet-water content is the ratio of the weight of seawater in the sample to the weight of the wet saturated sample, and is expressed as a percentage. Porosity is the ratio of the pore volume in a sample to the volume of the wet saturated sample, and is expressed as a percentage in some cases and as a fraction in others. All of these equations, derivations, and techniques are discussed in detail in Boyce, this volume.

\section{METHODS}

The following technique was used for sedimentary samples. Generally, in the Glomar Challenger laboratories, an undisturbed (visibly undistorted bedding), wet-saturated sample was cut and removed from a split core liner after the core had been on deck for about 4 hours to allow it to approach room temperature. The sample was then carefully cut (if necessary) with a diamond saw and smoothed with a sharp knife or file to a D-shaped sample $2.5 \mathrm{~cm}$ thick and with a 2.5 - $\mathrm{cm}$ radius. Compressional-wave (sound) velocities $( \pm 2 \%)$ perpendicular and parallel to bedding were measured with the Hamilton Frame velocimeter (Boyce, 1976a, and Boyce, this volume). Immediately afterward, wet-bulk density was measured within \pm 2 or $3 \%$ using special two-minute gamma-ray counts with the Gamma-Ray Attenuation Porosity Evaluator (GRAPE) (Evans, 1965) as modified by Boyce (1976a, and this volume). Between various measurements, the sample was wrapped in plastic and stored in a sealed plastic box with a wet sponge so that it would not dry out. The wet-water content, wetbulk density, and porosity of a subsample were then determined by weighing the water-saturated sample in water and after drying for 24 hours at $110^{\circ} \mathrm{C}$. For the soft sediments at Hole 530B and Site 532, porosity-density was determined by the "cylinder technique." These were processed at DSDP. The weight of evaporated water was corrected for salt content $(35 \%$ ) to give the weight of seawater (Boyce, 1976a; Boyce, this volume). The estimated precision of wet-bulk density is $\pm 0.01 \mathrm{~g} / \mathrm{cm}^{3}$ (absolute), and the precision of wet-water content and porosity is $\pm 0.5 \%$ absolute units. Acoustic impedance, in units of $\left(\mathrm{g} \cdot 10^{5}\right) /\left(\mathrm{cm}^{2} \cdot \mathrm{s}\right)$, is obtained from the product of the vertical (if possible) velocity and the gravimetric (if possible) wet-bulk density. Laboratory results are reported in tables in the site summaries.

For basalts, velocities were measured when the basalt first arrived in the laboratory; this allowed us to be certain that the sample was saturated with water. Detailed methods are discussed in Boyce (this volume). All basalt GRAPE 2-minute wet-bulk densities, and gravimetric wet-bulk densities, wet-water contents, and porosities were determined on minicores, using techniques identical to those employed for hard sedimentary material.

In situ velocity and electrical resistivity were obtained from Gearhart-Owen well-log combinations: (1) Compensated Sonic Log, Caliper, and Gamma Ray, and (2) Induction, 16-Inch Normal and Gamma Ray. Tools and precautions regarding the data are discussed in Boyce (this volume). Only the Sonic and Induction Log data from 625 to $945 \mathrm{~m}$ in Hole 530A will be discussed in this chapter. See the site summary for further discussion and other logging data.

With respect to the accuracy of logging data, I do not have absolute techniques available (e.g., in situ standards or in situ beds with precisely known in situ physical property values) with which to check 
empirically the validity of the logging data. The Velocity Log data appear to be low $(7-15 \%)$ when compared to laboratory velocities (see site summary, this volume), particularly where the hole is washed out. This is a common problem between logging velocity and ultrasonic velocity, measurements on core samples; for example, Jones and Wang (1981) partially attribute discrepancies to the following possibilities: (1) short spacing well logging tools, (2) attenuation, (3) physically disturbed borehole walls, and (4) biased core recovery, in that more resistant higher velocity rocks and softer material may have been eroded away during coring.

Because we do not have any absolute method by which to evaluate the logging data, any log-derived relationships between electrical resistivity, velocity, and density-porosity are subject to bias if the logging tools are not working properly.

\section{Electrical Resistivity}

The electrical resistivity of a material is defined as the resistance, in ohms, between opposite faces of a unit cube of that material. If the resistance of a conducting cube with length $L$ and cross-sectional area $A$ is $r$, then the resistivity, $R_{o}$, is

$$
R_{o}=r A / L=\text { ohm }-\mathrm{m}
$$

Electrical conduction through saturated sediment is complicated by a framework that generally consists of nonconducting mineral grains. If the sediment consists of nonconducting minerals, electrical conduction is primarily through the interstitial water, whose conductivity varies with temperature, salinity, and pressure (Horne, 1965; Horne and Courant, 1964; Horne and Frysinger, 1963; Thomas et al., 1934). Conduction through the fluid can be modified significantly, however, if there are present metallic minerals with appreciable conductivity or clay-type minerals that exchange or withdraw ions from the interstitial water (de Witte, 1950a, b; Patnode and Wyllie, 1950; Keller, 1951; Berg, 1952; Winsauer and McCardell, 1953; Wyllie, 1955). Charged colloidal particles and exchanged ions are not necessarily removed from the sediment when the interstitial water is sampled, so they do not contribute to what is normally thought of as the water salinity (Keller, 1951; Howell, 1953).

The formation factor, $F$, is the ratio of the electrical resistivity of the saturated sediment, $R_{o}$, to the resistivity of the interstitial water, $R_{w}$, at the same temperature and pressure (Archie, 1942):

$$
F=R_{o} / R_{w}
$$

The formation factor has been related to porosity and fluid salinity of rocks or sediments by Archie (1942; 1947), Winsauer et al. (1952), and others (Appendix A).

If the mineral composition of the sediment forms a nonconductive matrix, and if the interstitial water conductivity is high, then this ratio is considered to be the "true" formation factor. With increasing salinity of the interstitial water, this "true" formation factor approaches a constant value for a given porosity and rock sample (Patnode and Wyllie, 1950; Keller and Frischknecht, 1966).

If sediments contain minerals which are conductors, then this ratio is considered to be an "apparent" forma- tion factor and is less than the "true" formation factor of sediments for a given set of porosity, textural, and cementation characteristics. The "apparent" formation factor approaches a constant value with different salinities, at given porosity, only if the conductivity of the interstitial water is much greater than that of the conducting minerals (Berg, 1952; Howell, 1953; Wyllie and Southwick, 1954; Wyllie, 1955).

The variation of the apparent formation factor with interstitial water resistivity may be related in part to the distribution of conducting grains in a sample. Wyllie and Southwick (1954) developed a model showing that the connected conducting grains are conductors in parallel with, and isolated conducting grains are conductors in series with, the interstitial fluid. If the interstitial fluid is a good conductor, all the conducting grains will contribute to the overall conduction. If the interstitial fluid is a moderate or poor conductor, the conducting grains in series with interstitial water will contribute a reduced proportion of the overall conduction of the rock matrix; thus, the formation factor appears to increase as the resistivity of the fluid increases.

Clay-type minerals with varying exchange capacities may act as resistors or conductors relative to different interstitial water resistivities. Because of the clay-type minerals and other possible conducting minerals, the formation factor (for a given sample) may not be constant for different interstitial water resistivities (Keller, 1951; Wyllie, 1955; Berg, 1952; Wyllie and Gregory, 1953; Winsauer et al., 1952; Winsauer and McCardell, 1953; Wyllie and Southwick, 1954; Keller and Frischknecht, 1966).

The resistivity of interstitial water may be estimated by measuring the resistivity of the water squeezed from the geologic sample or by taking it to be equal to the resistivity of seawater. However, interstitial-water sampling may not remove ions that are filtered or trapped by clay-type minerals (Scholl, 1963), and the natural sediment compaction from overburden pressure may trap or filter various ions as the fluid migrates; thus, the interstitial fluid may have a chemical composition different from that of the original interstitial seawater (Siever et al., 1961; Siever et al., 1965). The electrical resistivity of the interstitial water determined, for example, by using the data of Thomas et al. (1934) may therefore be in error, because their data apply to a chemical composition identical to that of seawater.

Electrical resistivity through fresh sediment may be isotropic (Bedcher, 1965), but consolidated sediments and rocks have anisotropic resistivities. Resistivity parallel to bedding is typically less than the resistivity perpendicular to bedding (Keller, 1966; Keller and Frischknecht, 1966).

Textures of the individual mineral grains affect electrical resistivity. The more angular textures create a longer path length through the sediment and thus a higher resistivity and a higher formation factor for a given porosity (Wyllie and Gregory, 1953). The resistivity is also affected by grain-size distribution, particularly for clay-type minerals. A finer grain size gives a greater surface area with ionic exchange capacity and so 
increases the number of ionic-cloud conductors in a given sample. This is also true, to a lesser degree, of nonclay-type minerals, such a quartz and feldspar (Keller and Frischknecht, 1966).

We will interpret the DSDP Hole 530A sonic and electrical logs by a technique developed by the petroleum industry (Schlumberger, 4, 1972) called the "apparent electrical resistivity of the interstitial water" ( $R_{w a}$ curve). (Normally a density log is used, but we did not get a successful density-logging run at these sites.) This technique will here involve calculating the porosity from the Sonic Log's velocity, based on the following empirical equation derived from laboratory velocityporosity data from cores taken within the same depth interval in the hole (625 to $945 \mathrm{~m}$ ):

$$
\phi=\sqrt[0.527]{\frac{1.33}{V}}
$$

where $\phi=$ fractional porosity and $V=$ velocity $(\mathrm{km} / \mathrm{s})$ from Sonic Log. Then, by using a simplified form of Archie's (1942) equation for the Site 530 data:

$$
\begin{gathered}
F=R_{o} / R_{w a}=\phi^{-m}=\frac{1.0}{\phi^{2}} \\
R_{w a}=R_{a} \phi^{2}
\end{gathered}
$$

By substituting in Equation 5 the "apparent formation resistivity" $\left(R_{a}\right)$ (not corrected for borehole diameter, borehole fluids, or the thicknesses of beds with contrasting resistivity) from the Induction Log (measures in direction which is parallel to bedding) and the $\phi$ derived from the Sonic Log, we can then solve for $R_{w a}$.

If the formation is homogeneous calcareous ooze with a uniform pore-water salinity and a uniform and normal temperature gradient, the " $R_{w a}$ versus depth" plot will theoretically be a straight line, but $R_{w a}$ will decrease slightly because of increasing temperature with increasing depth. The method is useful because $R_{w a}$ will be anomalously high if there are any unexpected zones (which can sometimes be very distinct) of (1) hydrocarbons, (2) relatively fresh water in the pores, or (3) negative-temperature anomalies. The $R_{w a}$ curve will give anomalously low values if there are any unexpected zones of (1) electrical conductors (metallic deposits), (2) relatively saltier pore waters, or (3) high-temperature anomalies. Since the composition of the pore fluids is known from samples of the sedimentary rocks collected on the Challenger (Gieskes, this volume), and we know the temperature of the formation, we therefore know what range of $R_{w a}$ to expect and should thus be able to identify the anomalies. If hydrocarbons are present, the approximate pore-water saturation, $S_{w}$, equals $\left(R_{w a}\right.$ expected $/ R_{w a}$ anomaly $)^{1 / 2}$ when using Equation 4.

\section{Sound Velocity}

Compressional-sound velocity in isotropic material has been defined (Wood, 1941; Bullen, 1947; Birch, 1961; Hamilton, 1971) as

$$
V=\left(\frac{\varkappa+4 s / 3}{\varrho_{b}}\right)^{1 / 2}
$$

where $V$ is the compressional velocity; $\varrho_{b}$ is the wet-bulk density in $\mathrm{g} / \mathrm{cm}^{3}$ and $\varrho_{b}=\varrho_{w} \phi+(1-\phi) \varrho_{g}$ (here $\phi$ is the fractional porosity of the sediment or rock and the subscripts $b, g$, and $w$ represent the wet-bulk density, grain density, and water density, respectively); $x$ is the incompressibility or bulk modulus; and $s$ is the shear (rigidity) modulus.

Where samples are anisotropic, $x$ and $s$ may have unique values for the corresponding vertical and horizontal directions. See Laughton (1957); Carlson and Christensen (1977); Gregory (1977); and Bachman (1979) for discussions of anisotropy.

Compressional velocity of sediments and rocks has been related to the sediment components by Wood (1941), Wyllie et al. (1956), Nafe and Drake (1957), and others, whose equations are listed in Appendix B. These will be discussed later. Velocity is related to mineralogical composition, fluid content, water saturation of pores, temperature, pressure, grain size, texture, cementation, direction with respect to bedding or foliation, and alteration, as summarized by Press (1966). Recently, Hamilton (1978) has summarized velocity-density relationships of sediment and rock of the seafloor. Christensen and Salisbury (1975) have summarized velocity-density relationships of basalt under pressure.

Basalt velocities at one atmosphere pressure have been published for cores recovered on Leg 37 (Hyndman, 1977); Leg 46 (Matthews, 1979), and Legs 51, 52, 53 (Salisbury et al., 1980; Donnelly et al., 1980; Hamano, 1980); Boyce (1981), and others.

We did not have density log data, which are normally used with sonic log data to calculate acoustic impedance. Therefore, in order to calculate acoustic impedance, we empirically calibrated the velocity from the Sonic Log, using equations derived from velocity-impedance measurements from cores. This calibration is based on cross-plots of laboratory-measured velocity versus laboratory-measured impedance; measurements were made on cores in the same depth interval in Hole $530 \mathrm{~A}$ as were the logging data (625 to $945 \mathrm{~m})$. The following empirical equation was derived:

$$
I=-1.9 \frac{\mathrm{g} \cdot 10^{5}}{\mathrm{~cm}^{2} \cdot \mathrm{s}}+\left(3.0 \frac{\mathrm{g}}{\mathrm{cm}^{3}}\right)(V)
$$

where $V$ is velocity $(\mathrm{km} / \mathrm{s})$ and $I$ is acoustic impedance, $\left(\mathrm{g} \cdot 10^{5}\right) /\left(\mathrm{cm}^{2} \bullet \mathrm{s}\right)$. Therefore by substituting velocity $(\mathrm{km} /$ s) from the Sonic Log into Equation 7, we could calculate acoustic impedance. However, Equation 7 should not be used for any other universal purpose beyond the calibration of these logging data. From this Sonic Logderived impedance data, Sonic-Log derived reflection coefficients (R.C.) were calculated:

$$
\text { R.C. }=\frac{I_{1}-I_{o}}{I_{1}+I_{o}}
$$


where $I_{o}$ is a rolling average of impedance 0.5 meter above the plotted reflection-coefficient data point, and $I_{1}$ is a rolling average of impedance for $0.5 \mathrm{~m}$ below the plotted reflection-coefficient data point.

Reflection coefficients (from 0 to $1121 \mathrm{~m}$ ) are also calculated from the laboratory-measured velocity and impedance data (see raw data in tabular form in the site summaries, this volume). These are done very simply by using the upper and lower impedance values as they are listed in their tables, and plotting the reflection coefficient value at the same depth as the lower impedance value (except for the seawater/seafloor interface). Because of this very simple approach, investigators must be careful about precisely correlating the laboratory-derived reflection coefficients to their seismic profiles.

Calculations of in situ velocities from laboratorymeasured velocities on cores are corrected for (1) hydrostatic pressure and in situ temperature, and (2) hydrostatic pressure, in situ temperature, plus porosity rebound (Hamilton, 1976), expansion after overburden pressure is released. The two possible values for in situ velocity are calculated, since the porosity rebound has not been completely proven. Techniques for calculating in situ velocities are discussed in Boyce (1976b). These data are presented in Tables 1 and 2 . They assume a 5\% (absolute units) porosity rebound for all rock $>30 \%$ porosity; a $2.5 \%$ rebound for rocks with porosities between 20 and $30 \%$; and no rebound for rocks with porosities less than $20 \%$. They do not include corrections for rigidity, which is created by grain-to-grain overburden pressure (Hamilton, 1965).

\section{Vane Shear Strength}

Shear strength of a soil or sediment mass is the summation of the forces of friction, cohesion, and bonding which combine to resist failure by rupture along a slip surface or by excessive plastic deformation under applied stresses (Moore, 1964). Shear strength is a complex property which is also related to the rate of shearing, the manner and rate of stress application, mineralogy (clay type), cementation, grain-size distribution and packing, sample disturbance, pore pressure, permeability and drainage of the pore water during shearing (Richards, 1961; Moore, 1964; Wu, 1966; Scott and Schoustra, 1968; Lambe and Whitman, 1969; Kravitz, 1970; and others).

According to Richards (1961) and Kravitz (1970), the following shear failure theory is the Coulomb (1776) failure equation as modified by Hvorslev $(1936 ; 1937)$. Shear strength $\left(\mathrm{g} / \mathrm{cm}^{2}\right)$ of a sediment at failure, $\tau_{\mathrm{f}}$, is as follows:

$$
\tau_{f}=c+(\sigma-\mu) \tan \phi
$$

where $c=$ cohesion, $\mathrm{g} / \mathrm{cm}^{2} ; \sigma=$ normal stress on the plane of failure, $\left(\mathrm{g} / \mathrm{cm}^{2}\right) ; \mu=$ excess pressure in pore water, $\mathrm{g} / \mathrm{cm}^{2} ; \phi=$ angle of internal friction, and $(\sigma-\mu)$ $=$ effective stress, $\mathrm{g} / \mathrm{cm}^{2}$. Equation 9 has two components: cohesion, $c$, and friction, $(\sigma-\mu) \tan \phi$. As summarized by Hamilton (1971), shear strength in sands, without significant amounts of fine silt and clay are defined by the friction component (i.e., these are cohesionless sediments). Most silt-clay sediments have both cohesion and friction (under normal stress). A few clays may have no angle of internal friction, in which case the shear strength is defined by cohesion alone.

$$
\tau_{f_{\text {clay }}}=c
$$

According to Kravitz (1970), in studies involving completely saturated clays of low permeability, such as those found in ocean environments, shear strength is usually obtained under conditions of no change in water content. This procedure is called undrained or quick testing. During undrained (quick) testing, the normal stress is zero, and the saturated sediment then behaves with respect to the applied stresses at failure as a purely cohesive material with an angle of shearing resistance equal to zero. When these conditions are met the equation for shear strength is expressed as $\tau_{f}=\mathrm{c}$.

However, according to Moore (1964), Equation 8 is used mainly as a simplified relationship, and for the convenience of calculating engineering properties of soils, it is generally understood that actual isolation of the cohesional and frictional components of sediments is theoretically unrealistic.

The relationships of Equations 9 and 10 to undrained shear strength in saturated clayey sediments are discussed by Schmertmann and Osterberg (1960), Richards (1961), Wu (1966), Hamilton (1971), Kravitz (1970), and others. Lambe (1960) discusses the shear strength of coarse sediments with respect to the additive relationships of cohesion, friction, interference, and dilatancy.

The following are some examples of the physical changes which may occur in a sediment sample when it shears: (1) the sample may expand or contract depending on the grain-size distribution and packing structure; (2) the shearing stress may be in part directed on the pore water trapped in the sediment if the sample is very fine grained and impermeable (undrained sample); (3) or shearing force may be entirely directed on the grainto-grain structure if the sample's grain size is large and the sample is highly permeable, allowing the water to drain (drained sample); (4) if a sample is moderately permeable, then the shear strength will be in part related to (a) the rate at which the shearing stress is applied, and (b) the rate which the pore water drains out of the sample.

For vane shear measurements in this chapter, a finegrained sample was selected so that permeability is low enough that the sample is assumed to be "undrained" (unless the core samples are gassy) during the shear test. To enhance this relationship the vane shear speed must be very rapid (Lambe and Whitman, 1969; Scott and Schoustra, 1968) and thus the DSDP vane shear device is set at $89^{\circ}$ of torque per minute (compared with the typical $6^{\circ}$ per minute suggested in ASTM, 1975). These shear strength measurements are conducted under laboratory pressures and temperatures.

An attempt was made to obtain an undisturbed sample. A criterion for disturbance is visibly undistorted bedding, although a truly undisturbed sample does not 


\section{R. E. BOYCE}

Table 1. Laboratory sound velocity and calculated in situ velocity, Hole 530A.

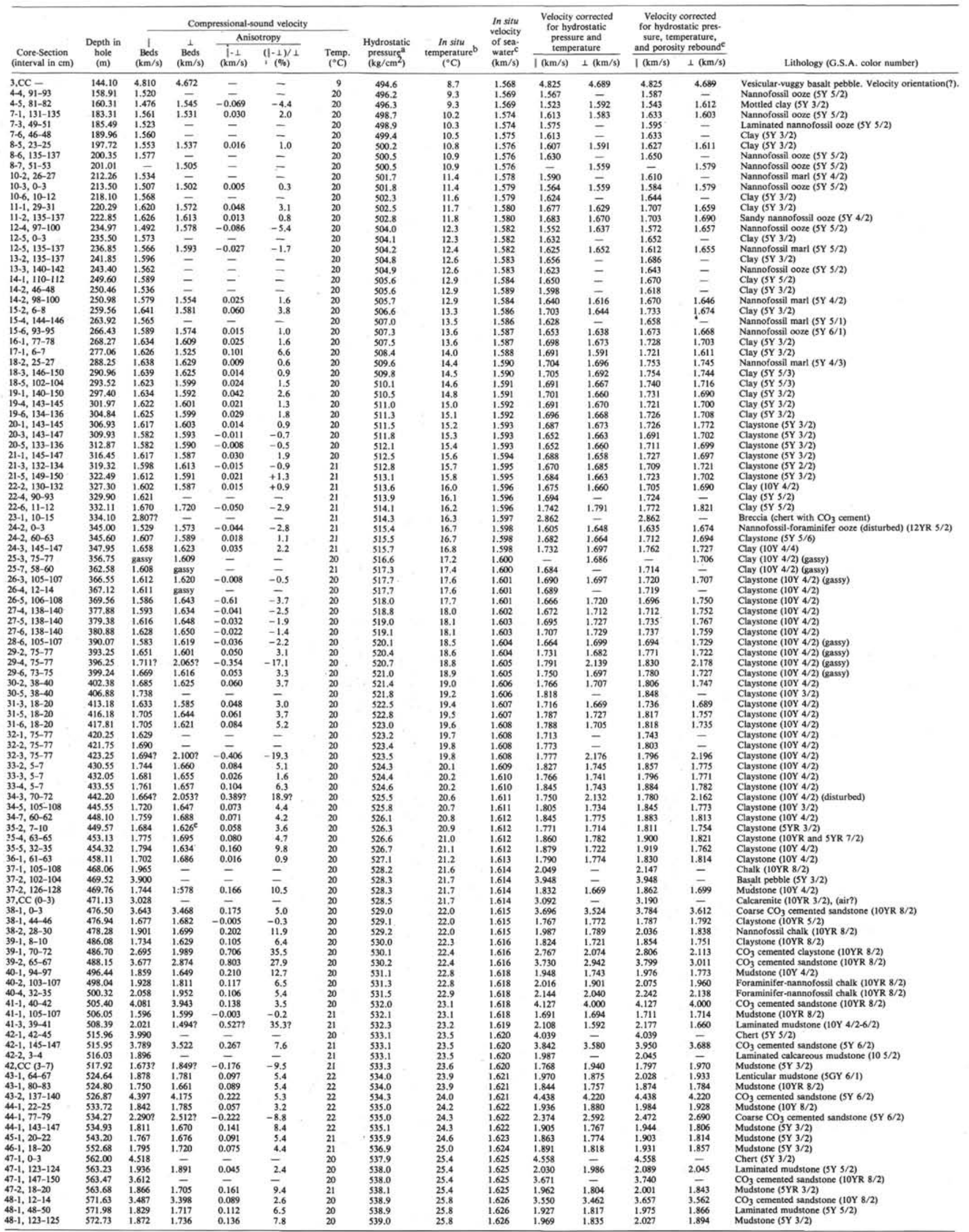


Table 1. (Continued).

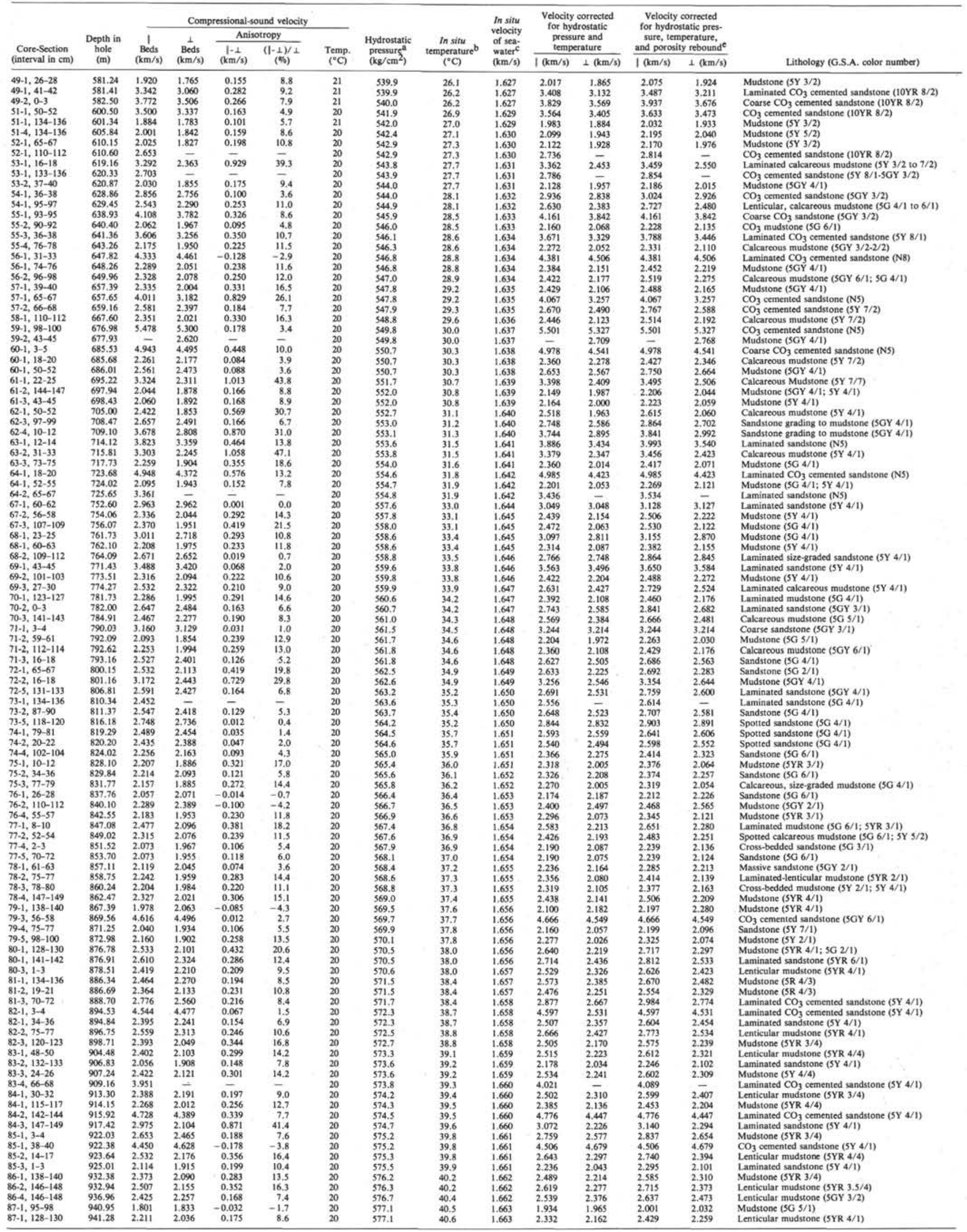


Table 1. (Continued).

\begin{tabular}{|c|c|c|c|c|c|c|c|c|c|c|c|c|c|c|}
\hline \multirow{3}{*}{$\begin{array}{c}\text { Core-Section } \\
\text { (interval in } \mathrm{cm} \text { ) }\end{array}$} & \multirow{3}{*}{$\begin{array}{c}\text { Depth in } \\
\text { hole } \\
(\mathrm{m})\end{array}$} & \multicolumn{5}{|c|}{ Compressional-sound velocity } & \multirow{3}{*}{$\begin{array}{c}\text { Hydrostatic } \\
\text { pressurfer } \\
\left(\mathrm{kg} / \mathrm{cm}^{2}\right)\end{array}$} & \multirow{3}{*}{$\begin{array}{c}\text { In situ } \\
\text { temperature } \\
\left({ }^{\circ} \mathrm{C}\right)\end{array}$} & \multirow{3}{*}{$\begin{array}{l}\text { In situ } \\
\text { velocity } \\
\text { of sea- } \\
\text { water } \\
\text { (km/s) }\end{array}$} & \multirow{2}{*}{\multicolumn{2}{|c|}{$\begin{array}{l}\text { Velocity corrected } \\
\text { for hydrostatic } \\
\text { pressure and } \\
\text { temperature }\end{array}$}} & \multirow{2}{*}{\multicolumn{2}{|c|}{$\begin{array}{c}\text { Velocity corrected } \\
\text { for hydrostatic pres. } \\
\text { sure, temperature. } \\
\text { and porosity rebounde }\end{array}$}} & \multirow[b]{3}{*}{ Lithology (G.S.A. color number) } \\
\hline & & & & & tropy & & & & & & & & & \\
\hline & & $(\mathrm{km} / \mathrm{s})$ & $(\mathrm{km} / \mathrm{s})$ & $(\mathrm{km} / \mathrm{s})$ & $(\%)$ & ("C) & & & & ( $(\mathrm{km} / \mathrm{s})$ & $1(\mathrm{~km} / \mathrm{s})$ & $1(\mathrm{~km} / \mathrm{s})$ & $+(\mathrm{km} / \mathrm{s})$ & \\
\hline $\begin{array}{l}87-2,118-120 \\
88-1,18-20\end{array}$ & $\begin{array}{l}942.68 \\
949.18\end{array}$ & 2.045 & 1.858 & 0.195 & 10.5 & 20 & 577.3 & 40.6 & 1.663 & 2.171 & 1.989 & 2.229 & 2.048 & Mudstone (SYR 3/4) \\
\hline $\begin{array}{l}888-1,180-72 \\
88-1,72\end{array}$ & 949.70 & $\begin{array}{l}\begin{array}{l}2.2029 \\
3.675\end{array} \\
-\end{array}$ & $2 . \overline{812}$ & 0.863 & $\overline{30.7}$ & ${ }_{20}^{20}$ & 578.0 & $\begin{array}{l}40.9 \\
40.9\end{array}$ & 1.664 & $\begin{array}{l}2.155 \\
3.755\end{array}$ & 2.917 & $\begin{array}{l}2.223 \\
3.55\end{array}$ & 2917 & $\begin{array}{l}\text { Mudstone (SYR 3/2) } \\
\text { Dofomitic } 3 / 2 \text { ) }\end{array}$ \\
\hline $88-1,128-130$ & 950.28 & 2.469 & 2.411 & 0.058 & 2.4 & 20 & 578.1 & 40.9 & 1.664 & 2.584 & 2.527 & $\begin{array}{l}3.695 \\
2.690\end{array}$ & $\begin{array}{l}2.2917 \\
2.633\end{array}$ & $\begin{array}{l}\text { Dolomitic musstone (5YK 3/2) } \\
\text { Sandstone (5Y S/1) }\end{array}$ \\
\hline $\begin{array}{l}88, \mathrm{CCC}(2-4) \\
89-1,7-80\end{array}$ & 953.48 & 2.219 & 2.084 & 0.135 & 6.5 & 20 & 578.4 & 41.0 & 1.664 & 2.341 & 2.210 & 2.438 & 2.307 & $\begin{array}{l}\text { Sandstone } \\
\text { Lenticular mudstone is }\end{array}$ \\
\hline $\begin{array}{l}89.1,78-80 \\
89-2,105-107\end{array}$ & $\begin{array}{l}958.78 \\
960.55\end{array}$ & $\begin{array}{l}2.107 \\
2058\end{array}$ & $\begin{array}{l}1.949 \\
1.841\end{array}$ & 0.158 & 8.1. & 20 & 579.0 & 41.3 & 1.665 & 2.233 & 2.080 & 2.320 & 2.167 & Lenticular mudstone (SGY $6 / 1 ; 5 G 5 / 1$ ) \\
\hline $\begin{array}{l}89-2,105-107 \\
89-3,130-132\end{array}$ & 960.35 & $\begin{array}{l}2.008 \\
2.094\end{array}$ & $\begin{array}{l}1.841 \\
1.948\end{array}$ & $\begin{array}{l}0.217 \\
0.146\end{array}$ & $\begin{array}{l}11.8 \\
7.5\end{array}$ & ${ }_{20}^{20}$ & $\begin{array}{l}579.1 \\
579.3\end{array}$ & 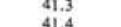 & 1.665 & 2.185 & 1.975 & 2.244 & 2.033 & Mudstone (SYR 3/2) \\
\hline $89.4,50-52$ & $\begin{array}{l}962.30 \\
963.00\end{array}-100$ & 2.047 & 1.857 & 0.190 & 10.2 & 20 & 579.4 & 年1.4.4 & 1.665 & 2.175 & $\begin{array}{l}2.079 \\
1.990\end{array}$ & $\begin{array}{l}2.288 \\
2.233\end{array}$ & $\begin{array}{l}2.147 \\
2.049\end{array}$ & $\begin{array}{l}\text { Calcareous mudstone (I } \\
\text { Mudstonc (SYR } 3 \text {, }\end{array}$ \\
\hline $90-1,45-47$ & 967.45 & 2.394 & 2.219 & 0.175 & 7.9 & 20 & 579.9 & 41.6 & 1.665 & 2.512 & 2.342 & $\begin{array}{l}2.233 \\
2.619\end{array}$ & $\begin{array}{l}2.0499 \\
2.449\end{array}$ & $\begin{array}{l}\text { Mudstone (SYR 3/4) } \\
\text { Mudstone (SY 3/4) }\end{array}$ \\
\hline $90-3,30-32$ & 970.30 & 1.951 & 1.813 & 0.138 & 7.6 & 20 & 580.1 & 41.7 & 1.666 & 2.082 & 1.948 & 2.141 & 2.007 & $\begin{array}{l}\text { Mustotone (SY } 3 / 4) \\
\text { Mudstone (SGY } 6 / 1 \text { ) }\end{array}$ \\
\hline $\begin{array}{l}91-1,140-142 \\
91-2,94-96\end{array}$ & 977.46 & 1,734 & 1.788 & -0.054 & -3.0 & 20 & 580.9 & 42.0 & 1.666 & 1.872 & 1.924 & 1.930 & 1.982 & Mudstone (SYR 4/1) \\
\hline $\begin{array}{l}91-2,294-96 \\
91-3,98-100\end{array}$ & $\begin{array}{l}978.44 \\
979.98\end{array}$ & 2.137 & $\begin{array}{l}1.990 \\
2.30\end{array}$ & 0.147 & 7.4 & 20 & 581.0 & 42.0 & 1.666 & $\begin{array}{l}2.263 \\
2.860\end{array}$ & 2.120 & 2.350 & 2.208 & Lenticular mudstone (5G $6 / 1$ ) \\
\hline & $\begin{array}{l}979.98 \\
980.62\end{array}-20$ & $\begin{array}{l}2.7722 \\
4.288\end{array}$ & $\begin{array}{l}2.3 .10 \\
3.692\end{array}$ & $\begin{array}{l}0.492 \\
0.596\end{array}$ & ${ }_{16 .}^{20.0}$ & 20 & $\begin{array}{l}581.2 \\
5812\end{array}$ & 年2,1 & $\begin{array}{l}1.667 \\
1.667\end{array}$ & $\begin{array}{l}2.880 \\
4.352\end{array}$ & $\begin{array}{l}2.432 \\
3,773\end{array}$ & 2.948 & 2.500 & Calcarcous mudstone (SYR $3 / 4$ ) \\
\hline $93-1,133-135$ & $\begin{array}{l}9801.32 \\
991.33\end{array}$ & 2.056 & $\begin{array}{l}3.005 \\
1.905\end{array}$ & 0.151 & $\begin{array}{l}10.9 \\
7.9\end{array}$ & ${ }_{20}^{20}$ & $\$ 582.3$ & 42.6 & $\begin{array}{l}1.007 \\
1.668\end{array}$ & 2.186 & 2.040 & $\begin{array}{l}4.352 \\
2.282 ?\end{array}$ & $\begin{array}{l}3.7733 \\
2.136 ?\end{array}$ & $\begin{array}{l}\text { Laminated } \mathrm{CO}_{3} \text { cemen } \\
\text { Mudstone (SYY } 3 / 2 \text { ) }\end{array}$ \\
\hline $93-2,72-74$ & 992.22 & 2.395 & 2.191 & 0.204 & 9.3 & 20 & 582.4 & 42.6 & 1.668 & 2.515 & 2.317 & 2.622 & 2.424 & Lenticular calcareous mudstone (SYR 4/4) \\
\hline $\begin{array}{l}93-3,60-62 \\
93-5,3-5\end{array}$ & 993.60 & 2.232 & 2.063 & 0.169 & 8.2 & 20 & $\begin{array}{c}582.6 \\
5828\end{array}$ & 42.6 & 1.668 & 2.357 & 2.193 & 2.415 & 2.251 & $\begin{array}{l}\text { Calcareous mudstone (SYR 4/4) } \\
\text { Lentitusure }\end{array}$ \\
\hline $94,1,11-13$ & $\begin{array}{l}996.03 \\
999.11\end{array}$ & $\begin{array}{l}2.1111 \\
2.750\end{array}$ & $\begin{array}{l}1.927 \\
2.084\end{array}$ & $\begin{array}{l}0.184 \\
0.666\end{array}$ & $\begin{array}{l}9.5 \\
32.0\end{array}$ & ${ }_{20}^{20}$ & $\begin{array}{l}582.8 \\
\$ 83.1\end{array}$ & ${ }_{42.9}^{42.7}$ & $\begin{array}{l}1.668 \\
1.669\end{array}$ & $\begin{array}{l}2.280 \\
2.860\end{array}$ & $\begin{array}{l}2.061 \\
2.214\end{array}$ & $\begin{array}{l}2.308 \\
2.919\end{array}$ & $\begin{array}{l}2,130 \\
2,272\end{array}$ & $\begin{array}{l}\text { Lenticular mudstone ( }(\mathrm{G} 4 / 1, \mathrm{~N} 8) \\
\text { Laminated mudstores (SG } 4 / 1)\end{array}$ \\
\hline $94-1,30-32$ & 999.30 & 2.081? & 2.283 & -0.202 & -8.8 & 20 & \$83.2. & $\begin{array}{l}42.9 \\
42.9\end{array}$ & 1.669 & 2.211 & $\begin{array}{l}2.214 \\
2.407\end{array}$ & 2.328 & $\begin{array}{l}2.272 \\
2.514\end{array}$ & 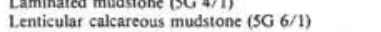 \\
\hline $94-2,140-142$ & 1001.90 & 2.063 & 1.891 & 0.172 & 9.1 & 20 & $\$ 883.4$ & $\begin{array}{l}42.9 \\
43.0\end{array}$ & 1.669 & 2.194 & 2.027 & 2.252 & 2.085 & Mudstone (SYR 3/2) \\
\hline $\begin{array}{l}995-1,102-104 \\
95-2,137-139\end{array}$ & 1009.02 & 2,186 & 1.933 & 0.253 & 13.1 & 20 & 584.2 & 43.3 & 1.670 & 2.314 & 2.069 & 2.382 & 2.137 & Mudstone (5YR 3/2) \\
\hline $\begin{array}{l}95-2,137-139 \\
95-3,118-120\end{array}$ & 1012.88 & $\begin{array}{l}2.2181 \\
2.300\end{array}$ & 2.201 & $\begin{array}{l}0.182 \\
0.159\end{array}$ & 9.1 & 20 & 584.3 & 43.3 & 1.670 & 2.309 & 2.133 & 2.406 & 2.230 & Laminated mudstone (SG 4/1; N-3) \\
\hline $96-1,73-77$ & 1017.73 & 1.881 & 2.115 & -0.234 & $\begin{array}{r}7.2 \\
-12.4\end{array}$ & 20 & 584.5 & $\begin{array}{l}43,4 \\
436\end{array}$ & 1.670 & 2.483 & $\begin{array}{l}2.329 \\
2.245\end{array}$ & $\begin{array}{l}2.590 \\
2.086\end{array}$ & 2.435 & 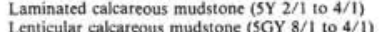 \\
\hline $96-2,75-77$ & 1019.25 & 2.276 & 2.198 & 0.078 & $\begin{array}{r}-12.4 \\
3.5\end{array}$ & $\begin{array}{l}20 \\
20\end{array}$ & $\begin{array}{l}5855.1 \\
585.2\end{array}$ & $\begin{array}{l}43.6 \\
437\end{array}$ & $\begin{array}{l}1.670 \\
1.670\end{array}$ & $\begin{array}{l}2.018 \\
2.401\end{array}$ & $\begin{array}{l}2.245 \\
2.326\end{array}$ & $\begin{array}{l}2.086 \\
2.508\end{array}$ & $\begin{array}{l}2.3 .33 \\
2.432\end{array}$ & 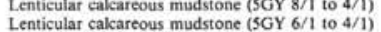 \\
\hline $96-2,98-100$ & 1019.48 & 2.045 & 1.899 & 0.146 & 7.9 & 20 & 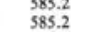 & $\begin{array}{l}43.7 \\
43.7\end{array}$ & $\begin{array}{l}1.670 \\
1.670\end{array}$ & $\begin{array}{l}2.401 \\
2.177\end{array}$ & $\begin{array}{l}2.326 \\
2.036\end{array}$ & $\begin{array}{l}2.2 .248 \\
2.245\end{array}$ & 2.104 & $\begin{array}{l}\text { Mudstone (SYR } 4 / 1 \text { ) } \\
\text { Mudstone (SOY } 6 / 1 \text { to } 4 / 1)\end{array}$ \\
\hline 97-1. 26-28 & 1026.26 & 4.421 & 4.382 & 0.039 & 0.9 & 20 & 585.9 & 44,0 & 1.671 & 4.482 & $\begin{array}{l}2.444 \\
4.440\end{array}$ & 4.482 & 4.4.44 & Lenticular calkareous mudstone (SCY 4/1; N3) \\
\hline $\begin{array}{l}97-3,5-7 \\
92-20\end{array}-18$ & 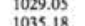 & 2.079 & 1.889 & 0.210 & 11.2 & 20 & 586.2 & 44.1 & 1.671 & 2.211 & 2.008 & 2.279 & 2.076 & Mudstone $(5 \mathrm{GY} 2 / 1)$ \\
\hline $98-2,20-22$ & 1036.70 & 2.100 & 1.806 & $\begin{array}{l}0.003 \\
0.194\end{array}$ & 4.4 & 20 & 586.9 & 44.3. & 1.672 & 2.1122 & $\begin{array}{l}2.032 \\
2004\end{array}$ & $\begin{array}{l}2.179 \\
2.300\end{array}$ & 2.099 & 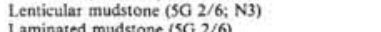 \\
\hline $98-3,10-12$ & 1038.10 & 2.165 & 1.974 & 0.191 & ${ }_{9.7}^{10.2}$ & ${ }_{20}^{20}$ & $\begin{array}{l}587.0 \\
587.2\end{array}-2$. & 每4.4.4. & $\begin{array}{l}1.672 \\
1.672\end{array}$ & $\begin{array}{l}2.232 \\
2.295\end{array}$ & $\begin{array}{l}2.044 \\
2.110\end{array}$ & $\begin{array}{l}2.300 \\
2.363\end{array}$ & $\begin{array}{l}2.1122 \\
2.178\end{array}$ & \\
\hline $99-1,138-140$ & 1045.38 & 1.863 & & - & - & 20 & 587.9 & $\begin{array}{l}44.4 \\
44.7\end{array}$ & 1.673 & 2.004 & - & $\begin{array}{l}2.363 \\
2.070\end{array}-100$ & 2.178 & $\begin{array}{l}\text { Laminated mimudstone }(5 G / 3) \\
\text { Mudstone }(5 Y 2 / 1)\end{array}$ \\
\hline $99-2,40-42$ & 1045.90 & 2.089 & 1.910 & 0.179 & 9.4 & 20 & 588.0 & 44,7 & 1.673 & 2.223 & 2.049 & 2.290 & 2.117 & Lenticular mudstone (5G 4/1; N3) \\
\hline $99-2,68-70$ & 1046.18 & 3.841 & 2.908 & 0.933 & 32.1 & 20 & 588.0 & 44.7 & 1.673 & 3.921 & 3.017 & 3.921 & 3.017 & Nannofossil limestone (5Y 4/1) \\
\hline & 1049.85 & $\begin{array}{l}2,170 \\
2.258\end{array}$ & 1.924 & $\begin{array}{l}0.246 \\
0.228\end{array}$ & 12.8 & 20 & 588.4 & 44.9 & 1.673 & 2.301 & 2.063 & 2.379 & 2.140 & Mudstone $(5 Y 2 / 1)$ \\
\hline $\begin{array}{l}100-1,140-142 \\
100.2,946\end{array}$ & $\begin{array}{l}1054.40 \\
1055.44\end{array}$ & $\begin{array}{l}2.228 \\
2.201\end{array}$ & $1.677 ?$ & $0.524 ?$ & $\begin{array}{ll}11.2 \\
31.27\end{array}$ & 20 & 588.9 & 45.1 & 1.674 & $\begin{array}{l}2.387 \\
2.332\end{array}$ & $\begin{array}{l}2.166 \\
1.824 ?\end{array}$ & $\begin{array}{l}2.455 \\
2.400\end{array}$ & 2.234 & 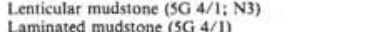 \\
\hline $\begin{array}{ll}100-3,34-36 \\
100-2,46\end{array}$ & 1056.34 & 2.334 & 2.169 & 0.165 & $\begin{array}{c}31.2 ? \\
7.6\end{array}$ & 20 & $\begin{array}{l}589.0 \\
5890\end{array}-1-20$ & $\begin{array}{l}45.1 \\
45.2\end{array}$ & $\begin{array}{l}1.674 \\
1.674\end{array}$ & $\begin{array}{l}2.332 \\
2.461\end{array}$ & 1.824? & $\begin{array}{l}2.400 \\
2.568\end{array}$ & 2.402 & 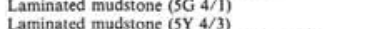 \\
\hline $100-4,36-38$ & 1057.86 & 2.271 & 2.073 & 0.198 & 9.6 & 20 & $\begin{array}{l}589.0 \\
589.2\end{array}$ & $\begin{array}{l}45.2 \\
45,2\end{array}$ & 1.674 & $\begin{array}{l}2.461 \\
2.400\end{array}$ & $\begin{array}{l}2.201 \\
2.208\end{array}$ & $\begin{array}{l}2.368 \\
2.497\end{array}$ & $\begin{array}{l}2.408 \\
2.395\end{array}$ & Laminated calcareous mudstone ( $5 G$ G $5 / 1$ ) \\
\hline $101-1,22-24$ & 1062.22 & 2.248 & 2.030 & 0.218 & $\begin{array}{l}9.6 \\
10.7\end{array}$ & 20 & $\begin{array}{l}589.7 \\
589.7\end{array}$ & $\begin{array}{l}4.2 .2 \\
45.4\end{array}$ & 1.675 & 2.378 & 2.167 & 2.455 & 2.244 & Mudstone ( $(5 Y 2 / 1)$ \\
\hline $101-2,33-35$ & 1063.83 & 2.295 & 2.137 & 0.158 & 7.4 & 20 & 589.8 & 45.5 & 1.675 & 2.424 & 2.271 & 2.491 & 2.339 & Laminated calcareous mudstone $(S \mathrm{SG} 6 / 1)$ \\
\hline $101-3,84-86$ & 1065.84 & 2.414 & 2.199 & 0.215 & 9.8 & 20 & (1) & 457 & 1.675 & 2.539 & 2.331 & 2.636 & 2.428 & Calcareous mudstone (5Y 5/1) \\
\hline $\begin{array}{l}101-5,136-138 \\
102-1,15-17\end{array}$ & 1069.36 & $\begin{array}{l}2.253 \\
2.336\end{array}$ & $\begin{array}{l}1.752 \\
2,154\end{array}$ & $\begin{array}{l}0.501 \\
0.232\end{array}$ & $\begin{array}{ll}28.6 \\
108\end{array}$ & 20 & $\begin{array}{l}590.4 \\
5906\end{array}$ & $\begin{array}{ll}45.7 \\
45.7\end{array}$ & 1.675 & $\begin{array}{l}2.383 \\
2.512\end{array}$ & 1,898 & 2.471 & 1.985 & $\begin{array}{l}\text { Mudstone (5YR 3/1) } \\
\text { Mudstong(5Y 2/1) }\end{array}$ \\
\hline $\begin{array}{l}102-1,1,1-17 \\
102-2,47-49\end{array}$ & $\begin{array}{l}1071.15 \\
1072.97\end{array}$ & $\begin{array}{l}2.3 .36 \\
2.246\end{array}$ & $\begin{array}{l}2.1 .144 \\
2.089\end{array}$ & 0.157 & $\begin{array}{l}10.8 \\
7.5\end{array}$ & 20 & $\begin{array}{l}590.6 \\
590.8\end{array}$ & $\begin{array}{l}45.7 \\
45.8\end{array}$ & $\begin{array}{l}1.675 \\
1.676\end{array}$ & $\begin{array}{l}2.2512 \\
2.377\end{array}$ & $\begin{array}{l}2.2 .27 \\
2.225\end{array}$ & $\begin{array}{l}2.473 \\
2.473\end{array}$ & $\begin{array}{l}2.334 \\
2.321\end{array}$ & $\begin{array}{l}\text { Mudatone (5Y } 2 / 1) \text {, } \\
\text { Mudstone (SGY 4/1): }\end{array}$ \\
\hline $\begin{array}{l}102-4,2-4 \\
\text { 10 }\end{array}$ & $\begin{array}{l}1075.52 \\
10729\end{array}$ & $\begin{array}{l}2.2460 \\
2.406\end{array}$ & $\begin{array}{l}2.1798 \\
2.175\end{array}$ & 0.231 & 10.6 & 20 & & 45 & 1.676 & 2.532 & 2.309 & 2.639 & 2.415 & $\begin{array}{l}\text { Laminated calcareous mudstone (SGY } 5 / 1) \\
\text { S }\end{array}$ \\
\hline $102-5,2-5$ & 1077.02 & 2.369 & 1.871 & 0.498 & 26.6 & 20 & 591.2 & 46.0 & 1.676 & 2.497 & 2.014 & 2.593 & 2.111 & Mudstone ( $(5 \times 2 / 1)$ \\
\hline $103-1,46-48$ & 1080.46 & & 2.096 & - & - & 20 & 59 & 46 & 1.676 & $-\bar{c}$ & 2.232 & -1 & 2.329 & Mudstone (s) \\
\hline $103-2,102-103$ & 1082.52 & 2.420 & 2.227 & 0.193 & 8.7 & 20 & 591.8 & 462 & i.t & 2.547 & 2,360 & 2.653 & $\begin{array}{l}2.466 \\
2,536\end{array}$ & $\begin{array}{l}\text { Lenticular ca } \\
\text { Mudstone is }\end{array}$ \\
\hline $\begin{array}{l}103-3,40-42 \\
103-4,2-4\end{array}$ & $\begin{array}{l}1083.40 \\
1034.50\end{array}$ & $\begin{array}{l}2.608 \\
2.44\end{array}$ & $\begin{array}{l}2.229 \\
2.226\end{array}$ & $\begin{array}{l}0.309 \\
0.218\end{array}$ & 13.4 .8 & ${ }_{20}^{20}$ & 5920 & $\begin{array}{l}46.2 \\
46.3\end{array}$ & 1.677 & $\begin{array}{l}2.779 \\
2.570\end{array}$ & $\begin{array}{l}2.430 \\
2.359\end{array}$ & 2.865 & $\begin{array}{l}2.536 \\
2.456\end{array}$ & $\begin{array}{l}\text { Mudstonec }(5 Y 3 / 1) \\
\text { Calcareous mudstone ( } 5 \mathrm{GG} 6 / 1 ; \mathrm{N} 3 \text { lenses) }\end{array}$ \\
\hline $104-1,120-122$ & $\begin{array}{l}1084.40 \\
1086.20\end{array}$ & $\begin{array}{l}2.444 \\
3.072\end{array}$ & $\begin{array}{l}2.2 .26 \\
2.86 \mathrm{I}\end{array}$ & $\begin{array}{l}0.218 \\
0.211\end{array}$ & $\begin{array}{l}9.8 \\
7.4\end{array}$ & ${ }_{20}^{20}$ & $\$ 92.0$ & 46.3 & $\begin{array}{l}1.677 \\
1.677\end{array}$ & $\begin{array}{l}2.570 \\
3.178\end{array}$ & $\begin{array}{l}2.3399 \\
2.974\end{array}$ & $\begin{array}{l}2.667 \\
3.265\end{array}$ & $\begin{array}{l}2.2466 \\
3.061\end{array}$ & 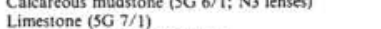 \\
\hline $104-2,100-102$ & 1087.50 & 2.370 & 2.120 & 0.250 & 11.8 & 20 & 592.3 & 46 & 1.677 & 2.498 & 2.256 & 2.595 & 2.353 & Mudstone ( $(5 \mathrm{G} 4 / 1 ; \mathrm{N} 3$ lenses) \\
\hline $104-3,38-40$ & 1088.38 & 2.463 & 2.194 & 0.269 & 12.3 & 20 & 592.4 & 46 & 1.677 & 2.588 & 2.328 & 2.695 & 2.434 & Laminated siltstone (5Y $9 / 1)$ \\
\hline $104-5,144-146$ & 1092.15 & 2.417 & 2.0 & 0. & 15.7 & 20 & & & & & & 2.642 & 2.324 & Mudstone $(5 Y 2 / 1)$ \\
\hline $\begin{array}{l}105-1,48-50 \\
105-3,18-20\end{array}$ & $\begin{array}{l}1094.48 \\
109718\end{array}$ & 2.363 & & 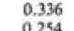 & 16.6 & 20 & & 46 & i. & $\begin{array}{l}2.492 \\
3,353\end{array}$ & 2.167 & 2.589 & 2.264 & $\begin{array}{l}\text { Lenticular mudstone }(5 \mathrm{GY} 4 / 1 ; \mathrm{N} 3) \\
\end{array}$ \\
\hline & $\begin{array}{l}1097.18 \\
1099.65\end{array}$ & $\begin{array}{l}3.2522 \\
3.053\end{array}$ & $\begin{array}{l}2.998 \\
2.832\end{array}$ & $0.22-2$ & $\begin{array}{l}8.5 \\
7.8\end{array}$ & ${ }_{20}^{20}$ & & $46-2$ & & $\begin{array}{l}3.333 \\
3.161\end{array}$ & $\begin{array}{l}3.947 \\
2.947 \\
-107\end{array}$ & 3.248 & 3.034 & $\left.\begin{array}{l}\text { Lenticular calcareous mudstone } \\
S \mathrm{G}\end{array} 6 / 1\right)$ \\
\hline $\begin{array}{l}105-5,-115-117 \\
1117\end{array}$ & $\begin{array}{l}1090.65 \\
1101.15\end{array}$ & $\begin{array}{l}3.0 .319 \\
2.319\end{array}$ & $\begin{array}{l}2.8832 \\
2.092\end{array}$ & $\begin{array}{l}0.227 \\
0.227\end{array}$ & $\begin{array}{c}7.8 \\
10.9\end{array}$ & 20 & & $46-2>$ & $\begin{array}{l}1.678 \\
1.678\end{array}$ & 2.450 & 2.230 & 2.556 & 2.336 & Mudstone (SYR 3.5/2) \\
\hline $106-1,8-10$ & 1103.08 & $3.813^{\mathrm{d}}$ & $2,05 z^{2}$ & 0.227 & 10.9 & $\operatorname{Cold}\left(15^{\circ} \mathrm{C}\right)$ & 593.9 & 40 & 1.679 & 3.897 & 2.250 & 3.897 & 2.000 & Basalt (velocity of whole \\
\hline $106-1,8-1$ & 1103.08 & $3.774^{\mathrm{d}}$ & $3.858^{\mathrm{d}}$ & -0.084 & -2.2 & 20 & 59 & 47 & i. & $-\overline{s e}$ & - & - & - & Basalt (vein) (vel \\
\hline 107-1, 12-14 & 1105.12 & 4.803 & & - & -38 & Cold & & 47 & 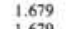 & 4.855 & - & 4.855 & - & $\begin{array}{l}\text { Basalt (velocity of whole core) } \\
\text {. }\end{array}$ \\
\hline $\begin{array}{l}107-1,12-14 \\
107-2,24-26\end{array}$ & $\begin{array}{l}1105.12 \\
1116.74\end{array}$ & $\begin{array}{l}4.693 \\
4.777\end{array}$ & 4.829 & -0.136 & -2.8 & $\begin{array}{c}20 \\
\text { Cold }\end{array}$ & 59 & 47. & 1.679 & $4 \overline{782}$ & $\bar{z}$ & $4 . \overline{782}$ & $\overline{-}$ & 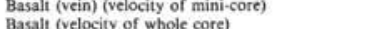 \\
\hline $\begin{array}{ll}107-2,224-26 \\
1072-24-26\end{array}$ & $\begin{array}{l}1106.74 \\
1106.74\end{array}$ & $\begin{array}{l}4.727 \\
4.711\end{array}$ & $4 . \overline{678}$ & $\overline{0.033}$ & $\overline{0.7}$ & Cold & 59 & 47. & if & 4.782 & - & ${ }_{-}^{4.782}$ & 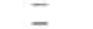 & $\begin{array}{l}\text { Bassint (velocity of whole core) } \\
\text { Basalt (velocity of mini-core) }\end{array}$ \\
\hline $107-3,41-43$ & 1108.41 & 4.924 & 4.618 & 0.033 & 0.7 & cold & 594.4 & 47. & 1.679 & 4.973 & $\bar{z}$ & $4 . \overline{973}$ & 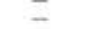 & Basalt (velocity of whole core) \\
\hline $107-3,41-43$ & 1108.41 & 5.013 & 5.030 & -0.017 & -0.3 & , & 594.4 & 47 & i. & & - & & - & Basalt (velocity of mini-core) \\
\hline 108-1, 19 & 1112 & 4.857 & 10 & - & - & cold & & 47 7 & 1. & 4.908 & - & 4.908 & - & Basalt (velocity of whole core) \\
\hline $\begin{array}{l}108-1,19 \\
108,28\end{array}$ & 1112.14 & $\begin{array}{l}4.94 \\
4.6\end{array}$ & 4.846 & 0.116 & 2.4 & 20 & $\begin{array}{l}594.8 \\
595.0\end{array}$ & $\begin{array}{l}47.7 \\
47.3-30\end{array}$ & 1.660 & $4 \overline{735}$ & $\bar{z}$ & $4 \overline{735}$ & 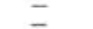 & $\begin{array}{l}\text { Bassalt (velocity of mini-core) } \\
\text { Basalt (velocity of whole core) }\end{array}$ \\
\hline $\begin{array}{l}108-2,58-6-60 \\
0108-2,58-60\end{array}$ & $\begin{array}{r}1114.08 \\
14.08\end{array}$ & $\begin{array}{l}4.658 \\
4,583\end{array}$ & $4 \overline{659}$ & $-0 . \overline{76}$ & $-\overline{1.6}$ & $\underset{20}{\text { Cold }}$ & 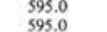 & 47. 47. & 1.680 & $\begin{array}{l}4.735 \\
-\end{array}$ & $\overline{-}$ & 4.735 & $\overline{-}$ & $\begin{array}{l}\text { Basalat (velocity of whole cores) } \\
\text { Basalt (velocity of mini-core }\end{array}$ \\
\hline $\begin{array}{l}108-2,38-60 \\
108-31-83\end{array}$ & $\begin{array}{r}114.08 \\
1115.83\end{array}$ & $\begin{array}{l}4.5833 \\
4.697\end{array}$ & 4.009 & -0.10 & -1.6 & $\begin{array}{l}20 \\
\text { Cold }\end{array}$ & $\begin{array}{l}595.0 \\
595.2\end{array}-2$ & $\begin{array}{l}47.5 \\
47.5\end{array}$ & 1.680 & $4 . \overline{753}$ & $=$ & $4 . \overline{753}$ & - & Basalt (velocity of whole core) \\
\hline $108-3,81-83$ & 1115.83 & 4.764 & 4.495 & 0.269 & 6.0 & 20 & 595.2 & 47.5 & 1.680 & -2 & $\bar{z}$ & 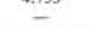 & $=$ & Basalt (vein) (velocity of mini-core) \\
\hline
\end{tabular}

${ }_{b}^{\text {a }}$ Hydrostatic pressure $=$ depth below sea level $\times 1.035 \mathrm{~g} / \mathrm{cm}^{3}$

6 Uses Navy SP58 with Tables. Linearly extrapolated from $35^{\circ} \mathrm{C}$.

d Uses the velocities measured through the whole basalt core for any velocity-related geophysical calculations. The velocities measured on the basalt minicores are used only to determine anisotropy, since these are not as accurate as the e whole-basalt core velocities.

exist. Vane shear measurements were formed adjacent to the sample for velocity, density, and porosity. Both sets of data appear to be of identical lithology.

On Leg 75, vane shear measurements were done with the DSDP Wykham Farrance Laboratory Vane Apparatus. All of the equipment, techniques, and calibrations are in Boyce (1977) and Boyce (this volume) and won't be discussed further here, except for changes from Boyce (1977) and other pertinent information. The 1.263 (high) $\times 1.278$ (diameter) $\mathrm{cm}$ vane was used, and it was buried about $0.7 \mathrm{~cm}$ on top and bottom of the sample. Because it was necessary to measure the shear strength on a split core (in order to find a proper lithologic sample), the vane was inserted parallel to bedding. The remolded test was done immediately after rotating the vane ten times (while in the sample).

Other DSDP investigators who have published vane shear strength are Lee (1973) and Rocker (1974); however, part of these samples are probably seriously disturbed. Keller and Bennett (1973) also measured an extensive number of shear strengths; however, the validity of their data is controversial since their cores were, in general, extremely disturbed.

Beginning with DSDP Leg 64, a hydraulic piston corer (HPC) was developed which can sample relatively undisturbed sediments. Therefore, the vane shear strengths presented in this chapter will be of specially selected, relatively undisturbed portions of these cores. 


\section{RESULTS}

The results apply to the laboratory-measured sound velocity, impedance, gravimetric porosity, gravimetric wet-bulk density, GRAPE two-minute wet-bulk density, shear strength, and their corresponding lithologies, which are in tabulated form in the site summaries (this volume). The in situ velocities, calculated from laboratory data, are in Tables 1 and 2. True formation electrical resistivity from the induction log and associated data are in Table 3. In general, the results apply to the following lithologic summary. Detailed discussions are in the site summaries (this volume).

\section{Lithologic Summary, Site $\mathbf{5 3 0}$}

From 0 to $110 \mathrm{~m}$ are Holocene to Pleistocene diatom nannofossil ooze and debris flows.

From 110 to $277 \mathrm{~m}$ are Pliocene to Miocene nannofossil clay, marl, ooze, and debris flows.

From 227 to $467 \mathrm{~m}$ are Miocene to Oligocene mudstone.

From 467 to $600 \mathrm{~m}$ are Eocene to Paleocene mudstone, marlstone, chalk, and clastic limestone.

From 600 to $704 \mathrm{~m}$ are Maestrichtian-Campanian mudstone, marlstone, clastic limestone, and calcareous siliclastic sandstone.

From 704 to $790 \mathrm{~m}$ are Campanian mudstone, marlstone, and calcareous siliclastic sandstone.

From 790 to $831 \mathrm{~m}$ are Campanian mudstone, marlstone and calcareous siliclastics sandstone.

From 831 to $940 \mathrm{~m}$ are Campanian to Santonian-Coniacian mudstone, claystone, siltstone, and sandstone.

From 940 to $1103 \mathrm{~m}$ are Santonian-Coniacian to late Albian-early Cenomanian claystone with interbedded black shale.

From 1103 to $1121 \mathrm{~m}$ are basalts.

\section{Lithologic Summary, Site $\mathbf{5 3 2}$}

From 0 to $217 \mathrm{~m}$ are Pleistocene to Pliocene diatom ooze, nannofossil ooze, marl, and clay.

From 217 to $292 \mathrm{~m}$ are late Miocene nannofossil ooze, marl, and clay.

The following scatter diagrams and figures are presented in order to provide empirical relationships, for comparison with previous or future studies, and to help develop predictive relationships.

The first scatter diagram (Fig. 2) shows gravimetrically determined wet-bulk density versus gravimetrically determined porosity for Sites 530 and 532 . On this diagram, the grain density of each sample may be estimated by a line from " $1.025 \mathrm{~g} / \mathrm{cm}^{3}$ (for $35 \%$ o salinity) density at $100 \%$ porosity" through "the given datum point" to the " $0 \%$ porosity axis." The grain density is the bulk density value at $0 \%$ porosity. This grain density determination is subject to great uncertainty, especially at high porosity, but at least it allows identification of sample data of questionable accuracy. Unusual grain density values could result from laboratory mistakes or from gas in the samples.

Figure 3 shows gravimetrically determined wet-bulk density versus wet-bulk density as determined by the
GRAPE 2-minute count. Considering all the assumptions of grain densities and attenuation coefficients, as discussed in Boyce (1976a), the correlation of the data is good.

Scatter diagrams of horizontal and vertical velocity are shown versus gravimetric porosity in Figure 4 and versus gravimetric wet-bulk density in Figure 5. These data are from Sites 530 and 532 and are coded for lithology. The average of the horizontal and vertical velocity versus gravimetric porosity (Fig. 6) and gravimetric wetbulk density (Fig. 7) are data from Hole 530A. Site 532 and Hole 530B did not have any vertical velocity measurements; therefore there is no such corresponding scatter diagram (sediment was too soft to measure vertical velocity). These figures are coded for lithology.

These figures illustrate the Wood (1941), Wyllie et al. (1956), and Nafe and Drake (1957) theoretical equations (listed in Appendix B), which utilize here, for simplicity, a calcium carbonate matrix $(6.45 \mathrm{~km} / \mathrm{s} ; 2.72 \mathrm{~g} /$ $\left.\mathrm{cm}^{3}\right)$ saturated with seawater $\left(1.53 \mathrm{~km} / \mathrm{s} ; 1.025 \mathrm{~g} / \mathrm{cm}^{3}\right)$. Wood's (1941) equation assumes a suspension of spheres without rigidity and theoretically best applies to soft unconsolidated sediment. This equation would tend to give the lower velocity limit. The Wyllie et al. (1956) equation assumes complete rigidity of the carbonate matrix and should theoretically give the upper velocity limit. The Nafe and Drake equation is shown for $n$ values of 4,6 , and 9. No single value of $n$ fits all the data. For some values of $n$, the velocities obtained from the Nafe and Drake (1957) equation may be too high (greater than the velocities from the Wyllie et al. equation) or too low (lower than the velocities from the Wood equation).

Acoustic impedance is plotted versus vertical velocity (laboratory) for Hole 530A in Figure 8. The plot approximates a linear relationship and normally segregates different mineralogies, such as basalt, clastics, limestone, and chert, into lines representing different bulk elasticities (Boyce, 1976b; Hamilton, 1976).

Acoustic anisotropy (Fig. 9) is important for estimating vertical velocities (for seismic reflection profiles) from (1) the horizontal velocities determined by refraction techniques, and (2) oblique velocities determined by sonobuoy techniques. Acoustic anisotropy in sedimentary rock may be created by some combination of the following variables, as summarized by Press (1966), Carlson and Christensen (1977), and Bachman (1979): (1) alternating layers with high- or low-velocity materials; (2) tabular minerals aligned with bedding, which create fewer gaps (containing pore water) in a direction parallel to bedding; (3) acoustically anisotropic minerals whose high-velocity axis may be aligned with the bedding plane; and (4) foliation parallel to bedding.

Absolute acoustic anisotropy versus depth at Hole $530 \mathrm{~A}$ is shown in Figure 10 and percentage acoustic anisotropy versus depth is shown in Figure 11.

The negative anisotropies from 350 to $400 \mathrm{~m}$ appear to be related to a gassy zone (gas is in the recovered cores and may not be in a gaseous state in situ); therefore, these negative anisotropies are probably not representative of in situ anisotropies. 
Table 2. Laboratory sound velocity and calculated in situ velocity, Hole 530B.

\begin{tabular}{|c|c|c|c|c|c|c|c|c|}
\hline \multirow[b]{3}{*}{$\begin{array}{c}\text { Core-Section } \\
\text { (interval in cm) }\end{array}$} & \multirow{3}{*}{$\begin{array}{l}\text { Depth in } \\
\text { hole } \\
\text { (m) }\end{array}$} & \multicolumn{5}{|c|}{ Compressional-sound velocity } & \multirow{3}{*}{$\begin{array}{c}\text { Hydrostatic }^{\mathrm{a}} \\
\text { pressure } \\
\left(\mathrm{kg} / \mathrm{cm}^{2}\right)\end{array}$} & \multirow{3}{*}{$\begin{array}{c}\text { In situ } \\
\text { temperature } \\
\left({ }^{\circ} \mathrm{C}\right)\end{array}$} \\
\hline & & \multirow{2}{*}{$\underset{\substack{\text { Beds } \\
(\mathrm{km} / \mathrm{s})}}{\mid}$} & \multirow{2}{*}{$\underset{\substack{\perp \\
(\mathrm{km} / \mathrm{s})}}{\stackrel{\perp}{\text { Beds }}}$} & \multicolumn{2}{|c|}{ Anisotropy } & \multirow[b]{2}{*}{$\begin{array}{l}\text { Temp. } \\
\left({ }^{\circ} \mathrm{C}\right)\end{array}$} & & \\
\hline & & & & $\underset{(\mathrm{km} / \mathrm{s})}{\|-\perp}$ & $\begin{array}{c}\left(\mid-\frac{1}{(\%)}\right) \\
(\%)\end{array}$ & & & \\
\hline $3-2,113-115$ & 10.93 & 1.506 & - & - & - & 21 & 480.9 & 3.3 \\
\hline $7-3,83-85$ & 27.23 & 1.489 & - & - & - & 21 & 482.5 & 4.0 \\
\hline $9-2,133-135$ & 35.03 & 1.585 & - & - & - & 21 & 483.3 & 4.3 \\
\hline $10-1,140-141$ & 38.00 & 1.482 & - & - & - & 20 & 483.7 & 4.4 \\
\hline $11-2,140-142$ & 43.90 & 1.505 & - & - & - & 20 & 484.3 & 4.7 \\
\hline $12-3,10-12$ & 48.50 & 1.495 & - & - & - & 20 & 484.7 & 4.8 \\
\hline $14-2,10-12$ & 55.80 & 1.513 & - & - & - & 20 & 485.5 & 5.1 \\
\hline $16-2,130-132$ & 65.80 & 1.503 & - & - & - & 20 & 486.5 & 5.5 \\
\hline $17-2,125-127$ & 70.15 & 1.517 & - & - & - & 20 & 487.0 & 5.7 \\
\hline $18-1,135-136$ & 73.15 & 1.497 & - & - & - & 20 & 487.3 & 5.8 \\
\hline $20-3,25-27$ & 83.85 & 1.505 & - & - & - & 20 & 488.4 & 6.3 \\
\hline $21-2,25-27$ & 86.75 & 1.499 & - & - & - & 20 & 488.7 & 6.4 \\
\hline $25-2,130-133$ & 102.60 & 1.500 & - & - & - & 20 & 490.3 & 7.0 \\
\hline $27-2,10-13$ & 108.80 & 1.507 & - & - & - & 20 & 491.0 & 7.3 \\
\hline $33-1,75-77$ & 118.95 & 1.586 & - & - & - & 20 & 492.0 & 7.7 \\
\hline $35-2,125-127$ & 137.15 & 1.560 & - & - & - & 20 & 493.9 & 8.4 \\
\hline $36-3,20-22$ & 142.00 & 1.578 & - & - & - & 20 & 494.4 & 8.6 \\
\hline $37-1,55-57$ & 142.75 & 1.535 & - & - & - & 20 & 494.5 & 8.6 \\
\hline $41-3,75-77$ & 158.35 & 1.524 & - & - & - & 20 & 496.1 & 9.2 \\
\hline $44-2,85-87$ & 165.35 & 1.534 & - & - & - & 20 & 496.8 & 9.5 \\
\hline $46-2,10-12$ & 172.40 & 1.500 & - & - & - & 20 & 497.6 & 9.8 \\
\hline $47-2,62-64$ & 176.32 & 1.538 & - & - & - & 20 & 498.0 & 10.0 \\
\hline $48-1,135-138$ & 178.95 & 1.520 & - & - & - & 20 & 498.2 & 10.1 \\
\hline
\end{tabular}

${ }^{\mathrm{a}}$ Hydrostatic pressure $=$ depth below sea level $\times 1.035 \mathrm{~g} / \mathrm{cm}^{3}$.

b Assumes $40^{\circ} \mathrm{C} / 1000 \mathrm{~m}$ temperature gradient for simplicity and seafloor temperature of $2.9^{\circ} \mathrm{C}$.

c Uses Navy SP58 with Table 5. Linearly extrapolated from $35^{\circ} \mathrm{C}$ to $48^{\circ} \mathrm{C}$ and assumes 35 ppt.

$\mathrm{d}$ These do not include corrections for changes in rigidity caused by overburden pressure.

From 100 to $467 \mathrm{~m}$, anisotropy irregularly increases to $10 \%$, with 2 to $5 \%$ being typical. From 467 to 1103 $\mathrm{m}$, anisotropies are as great as $47 \%(1.0 \mathrm{~km} / \mathrm{s})$. Mudstone and uncemented sandstone have anisotropies which irregularly increase with increasing depth from 5 to $10 \%$ $(0.2 \mathrm{~km} / \mathrm{s})$. Calcareous cemented mudstone tends to have the greatest anisotropies, typically $35 \%(0.6 \mathrm{~km} / \mathrm{s})$.

For Site 530, vertical velocity versus depth (except for Hole 530B, 0-175 meters, where only horizontal velocities were measured because the samples were too soft to measure vertical velocity) is displayed in Figure 12. In Figure 12, these velocities are at laboratory temperature and pressure, and are coded for lithology.

At $\sim 60$ to $\sim 70 \mathrm{~m}$, and at $\sim 110$ to $230 \mathrm{~m}$, these are several debris-flow deposits, which in some cases have a slightly higher velocity than the host sediments.

From 500 to $700 \mathrm{~m}$, the minimum mudstone velocities have increasing curve versus increasing depth. This is a function of soft mudstone densities, which have an approximately linear increase between 500 and $700 \mathrm{~m}$; thus the curved velocity trend is, in general, related to Wood's (1941) equation of velocity and density-porosity as in Figures 6 and 7 and Figures 4 and 5.

Many of the lithologic boundaries are characterized by changes in sound velocity-for example, at 110, 277, $600, \sim 700,790$, and $1103 \mathrm{~m}$. Many age boundaries occur at horizons of obvious changes in velocity, for example at $110 \mathrm{~m}$ for the Pleistocene/Pliocene boundary; at $420 \mathrm{~m}$ for the early Miocene/Oligocene boundary; at about $465 \mathrm{~m}$ at the Oligocene/Eocene boundary; at 600 $\mathrm{m}$ at the Paleocene/Maestrichtian boundary; and at 685 $\mathrm{m}$, which is near the Maestrichtian/Campanian boundary. Of course, these variations coincide with subtle variations in lithology.

In Figure 13 is shown vertical laboratory velocity (at laboratory temperature and pressure) versus depth. Also included are (1) laboratory velocities which are corrected for in situ temperature and hydrostatic pressure, and (2) laboratory velocities which are corrected for hydrostatic pressure, in situ temperature, and porosity rebound (expansion when overburden pressure is released). These values do not include corrections for rigidity caused by grain-to-grain overburden pressure (Hamilton, 1965). Porosity rebound corrections are theoretical (Hamilton, 1976) and have not been demonstrated to be true.

Averages for the velocity for Hole 530A have been calculated, and these results (with assumptions and other details) are published in the site summary (this volume). The averages in the upper $467 \mathrm{~m}$ of the hole agree fairly well with Sibuet's (see site summary, this volume) correlations to the seismic profile. For example, the uncorrected laboratory average velocity is $1.56 \mathrm{~km} / \mathrm{s}$, in situ corrected (not corrected for rigidity caused by overburden pressure) laboratory average velocity is $1.61 \mathrm{~km} / \mathrm{s}$, and Sibuet's average velocity is 1.59 $\mathrm{km} / \mathrm{s}$. In the lower portions of Hole 530A, 467 to 1103 $\mathrm{m}$, the laboratory averages do not agree with Sibuet's velocity; for example, the average of uncorrected laboratory average velocities is $2.15 \mathrm{~km} / \mathrm{s}$, the average of the in situ corrected laboratory velocity is $2.37 \mathrm{~km} / \mathrm{s}$, 
Table 2. (Continued).

\begin{tabular}{|c|c|c|c|c|c|}
\hline \multirow{2}{*}{$\begin{array}{c}\text { In situ } \\
\text { velocity } \\
\text { of seawater } \\
(\mathrm{km} / \mathrm{s})\end{array}$} & \multicolumn{2}{|c|}{$\begin{array}{l}\text { Velocity corrected } \\
\text { for hydrostatic } \\
\text { pressure and } \\
\text { temperature }\end{array}$} & \multicolumn{2}{|c|}{$\begin{array}{l}\text { Velocity corrected for } \\
\text { hydrostatic pressure, } \\
\text { temperature, and } \\
\text { porosity rebound } \\
\end{array}$} & \multirow[b]{2}{*}{ Lithology (G.S.A. color number) } \\
\hline & $\|(\mathrm{km} / \mathrm{s})$ & $\perp(\mathrm{km} / \mathrm{s})$ & $\|(\mathrm{km} / \mathrm{s})$ & $\perp(\mathrm{km} / \mathrm{s})$ & \\
\hline 1.544 & 1.528 & - & 1.528 & - & Clay $(5 Y 2 / 1)$ \\
\hline 1.546 & 1.513 & - & 1.513 & - & Clay $(5 \mathrm{GY} 4 / 1)$ \\
\hline 1.549 & 1.612 & - & 1.612 & - & Clay $(5 G Y 4 / 1)$ \\
\hline 1.550 & 1.510 & - & 1.510 & - & Nannofossil ooze $(5 Y$ Y $6 / 1)$ \\
\hline 1.551 & 1.534 & - & 1.534 & - & Clayey diatom ooze (5Y 3/1) \\
\hline 1.551 & 1.524 & - & 1.524 & - & Clayey diatom ooze (5Y $3 / 1)$ \\
\hline 1.552 & 1.543 & - & 1.543 & - & Diatom nannofossil ooze $(5 \mathrm{G} 6 / 1)$ \\
\hline 1.554 & 1.535 & - & 1.535 & - & Clayey diatom ooze $(5 Y 4 / 1)$ \\
\hline 1.555 & 1.550 & - & 1.550 & - & Clayey diatom ooze (5Y 3/1) \\
\hline 1.555 & 1.530 & - & 1.530 & - & Clayey diatom ooze (5Y 3/1) \\
\hline 1.558 & 1.541 & - & 1.561 & - & Clayey diatom ooze (5Y 3/1) \\
\hline 1.558 & 1.535 & - & 1.535 & - & Clayey diatom ooze (5Y 3/1) \\
\hline 1.560 & 1.538 & - & 1.538 & - & Clayey diatom ooze (marl) (5G 4/1) \\
\hline 1.562 & 1.547 & - & 1.547 & - & Mottled clayey diatom ooze (5Y $4 / 1)$ \\
\hline 1.563 & 1.626 & - & 1.626 & - & Mud flow clast: diatomaceous clay (5YR 3/1) \\
\hline 1.565 & 1.603 & - & 1.633 & - & Mudflow clast: nannofossil ooze (5Y 6/1) \\
\hline 1.567 & 1.622 & - & 1.642 & - & Mudflow clast: mottled clayey nannofossil ooze $(5 Y$ 6/1) \\
\hline 1.568 & 1.581 & - & 1.601 & - & Nannofossil ooze (5Y 6/1) \\
\hline 1.569 & 1.571 & - & 1.591 & - & Nannofossil ooze (5Y $6 / 1)$ \\
\hline 1.570 & 1.582 & - & 1.602 & - & Laminated nannofossil ooze (5Y 6/1) \\
\hline 1.572 & 1.550 & - & 1.550 & - & Mudflow clast: clay (5Y $2 / 1)$ (disturbed) $(5 Y 2 / 1)$ \\
\hline 1.573 & 1.589 & - & 1.609 & - & Mudflow matrix: nannofossil ooze (5G 8/1) (disturbed) (5G 6/1) \\
\hline 1.573 & 1.571 & - & 1.591 & - & Layered nannofossil ooze (5G 6/1) \\
\hline
\end{tabular}

and Sibuet's average velocity is $2.14 \mathrm{~km} / \mathrm{s}$. Sibuet's lower velocity depends on the upper basalt horizon correlating to the 1.2-s reflector; however, in the Challenger profile over Site 530 the basalt reflector is poor, and is either very weak or much higher in the profile and with significantly less than the 1.2-s reflection time used by Sibuet. Sibuet's 1.2-s reflector and seismic correlations are with another seismic profile, which does not prescisely cross (off by 2.5 miles) Site 530 . As a result of these conditions, Sibuet's correlation and velocities are subjective and perhaps controversial.

It is also possible that the average laboratory velocities are incorrect (as many assumptions are involved), or perhaps that the in situ corrections are not valid, for porosity rebound has not been proved. These velocity discrepancies will not be resolved here. To perhaps resolve this problem we need good seismic profiles which truly pass over Site 530, along with research to substantiate porosity rebound and additional studies of acoustic attenuation in these seismic profiles.

Horizontal uncorrected velocities of the laboratory samples are plotted versus depth for Site 530 (Fig. 14A) and Site 532 (Fig. 14B). These are coded for lithology. Figure 15 shows (1) the uncorrected laboratory horizontal velocities versus depth at Site 530; in addition, it also shows (2) laboratory velocities corrected to in situ temperature and hydrostatic pressure, and (3) laboratory velocities which are corrected for in situ temperature, hydrostatic pressure, and porosity rebound. These are not corrected for rigidity caused by overburden pressure.

Gravimetric wet-bulk density is plotted versus depth for Site 530 (Fig. 16A) and 532 (Fig. 16B), and gravi- metric porosity is plotted versus depth at Site 530 (Figure 17A) and Site 532 (Figure 17B). These are coded for lithology. These data show good agreement with the summary in Hamilton (1976) for density versus depth curves of terrigenous uncemented mudstone and uncemented siliceous and calcareous ooze. There are two zones of relatively higher porosity and relatively lower density than predicted by Hamilton's (1976) densityporosity versus depth curves (for terrigenous sediment): at approximately 325 to $500 \mathrm{~m}$ and at approximately 810 to $1025 \mathrm{~m}$; these could be zones where pore fluids are overpressurized, the result of low-permeability mudstone's preventing pore fluids from escaping as overburden pressure of the grains attempts to consolidate the sediment. However, these zones are probably related to variations in grain-size distribution and variations in lithology (Hamilton and Menard, 1956; Horn et al., 1969; Hamilton, 1980).

Cross plots of laboratory velocity $(V)$ versus acoustic impedance $(I)$ for the interval of 625 to $945 \mathrm{~m}$ in Hole 530A are shown in Figure 18. From these data, Equation 7 is derived:

$$
\mathrm{I}=-1.9\left(\mathrm{~g} \cdot 10^{5}\right) /\left(\mathrm{cm}^{2} \cdot \mathrm{s}\right)+\left(3.0 \mathrm{~g} / \mathrm{cm}^{3}\right)(V)
$$

Equation seven (7) is used to calculate impedance from the velocities measured by the Sonic Log from 625 to $945 \mathrm{~m}$ in Hole 530A. The Sonic Log derived acousticimpedance data and the reflection coefficient plots versus depth are shown in Figures 19 and 20. In Figures 19 and 20, the Sonic Log velocities are low compared to the velocities of the core samples, as discussed in the site summary, this volume. These velocities are more than 
Table 3. Electrical resistivity, formation factors, and sound velocity data from the well logs and other associated data, Hole 530A.

\begin{tabular}{|c|c|c|c|c|c|c|c|c|c|c|c|}
\hline \multirow{2}{*}{$\begin{array}{l}\text { Depth } \\
\text { from rig } \\
\text { floor } \\
\text { (m) }\end{array}$} & \multirow[b]{2}{*}{$\begin{array}{c}\text { Depth } \\
\text { below } \\
\text { seafloor }^{\mathrm{a}} \\
(\mathrm{m})\end{array}$} & \multirow[b]{2}{*}{$\begin{array}{c}\text { Depth } \\
\text { below } \\
\text { sea level }^{\mathrm{b}} \\
\text { (m) }\end{array}$} & \multirow[b]{2}{*}{$\begin{array}{c}\text { Hydrostatic } \\
\text { pressure } \\
\left(\mathrm{kg} / \mathrm{cm}^{2}\right)\end{array}$} & \multirow[b]{2}{*}{$\begin{array}{c}\text { Salinity } \\
\text { of pore } \\
\text { water }^{d} \\
(\%)\end{array}$} & \multirow[b]{2}{*}{$\begin{array}{l}\text { Temperature } e^{\mathrm{e}} \\
\left({ }^{\circ} \mathrm{C}\right)\end{array}$} & \multicolumn{2}{|c|}{$\begin{array}{c}\text { In situ } \\
\text { Pore water }\end{array}$} & & & \multicolumn{2}{|c|}{$\begin{array}{c}\text { True formation (hole and } \\
\text { bed thickness corr.) }\end{array}$} \\
\hline & & & & & & $\begin{array}{c}\text { Resistivity, } \\
R_{W} \\
\text { (ohm-m) }\end{array}$ & $\begin{array}{l}\text { Conductivity, } \\
C_{W} \\
(\mathrm{~m}-\mathrm{mhos} / \mathrm{m})\end{array}$ & $\begin{array}{l}\text { Bor } \\
\text { dian } \\
\text { (in.) }\end{array}$ & $\begin{array}{l}\text { hole } \\
\text { leter } \\
(\mathrm{cm})\end{array}$ & $\begin{array}{c}\text { Resistivity, } \\
R_{t} \\
\text { (ohm-m) }\end{array}$ & $\begin{array}{c}\text { Conductivity, } \\
C_{t} \\
(\mathrm{~m}-\mathrm{mhos} / \mathrm{m})\end{array}$ \\
\hline 5274 & 629 & 5264 & 544.8 & 34.1 & 28.1 & 0.1762 & 5677 & 11.3 & 28.7 & $2.00(?)$ & 500 \\
\hline 5282 & 637 & 5272 & 545.7 & 34.1 & 28.4 & 0.1707 & 5859 & 11.2 & 28.4 & 2.40 & 417 \\
\hline 5294.5 & 649.5 & 5284.5 & 546.9 & 34.1 & 28.9 & 0.1735 & 5761 & 11.2 & 28.4 & 3.05 & 328 \\
\hline 5307.5 & 662.5 & 5297.5 & 548.3 & 34.1 & 29.4 & 0.1720 & 5813 & 11.1 & 28.2 & 1.52 & 656 \\
\hline 5341 & 696 & 5331 & 551.8 & 34.1 & 30.7 & 0.1681 & 5949 & 11.3 & 28.7 & 1.54 & 651 \\
\hline 5356 & 711 & 5346 & 553.3 & 34.1 & 31.3 & 0.1664 & 6009 & 11.5 & 29.2 & 1.61 & 620 \\
\hline 5360 & 715 & 5350 & 553.7 & 34.1 & 31.5 & 0.1659 & 6029 & 11.3 & 28.7 & 1.37 & 737 \\
\hline 5366.5 & 721.5 & 5356.5 & 554.4 & 34.1 & 31.7 & 0.1653 & 6050 & 11.4 & 29.0 & 1.71 & 584 \\
\hline 5372 & 727 & 5362 & 555.0 & 34.1 & 32.0 & 0.1645 & 6080 & 11.2 & 28.4 & 2.50 & 400 \\
\hline 5382.5 & 737.5 & 5372.5 & 556.1 & 34.1 & 32.4 & 0.1634 & 6120 & 11.25 & 28.6 & 1.29 & 773 \\
\hline 5447.5 & 802.5 & 5437.5 & 562.8 & 34.1 & 35.0 & 0.1566 & 6386 & 11.0 & 27.9 & 2.90 & 345 \\
\hline 5454.5 & 809.5 & 5444.5 & 563.5 & 34.1 & 35.3 & 0.1558 & 6417 & 11.25 & 28.61 & 2.61 & 383 \\
\hline 5467 & 822 & 5457 & 564.8 & 34.1 & 35.8 & 0.1546 & 6468 & 11.15 & 28.3 & 1.73 & 578 \\
\hline 5523 & 878 & 5513 & 570.6 & 34.1 & 38.0 & 0.1493 & 6698 & 11.3 & 28.7 & $1.70(?)$ & 588 \\
\hline 5524 & 879 & 5514 & 570.7 & 34.1 & 38.1 & 0.1491 & 6708 & 10.8 & 27.4 & $1.40(?)$ & 714 \\
\hline
\end{tabular}

a Depth below rig floor (on logs) $-4645 \mathrm{~m}=$ depth below seafloor.

b Depth below sea level = depth below seafloor plus water depth $(4635 \mathrm{~m})$.

c Hydrostatic pressure $=$ depth below sea level $\times 1.035 \mathrm{~g} / \mathrm{cm}^{3}$.

d Salinity of $34.1 \mathrm{ppt}$ is extrapolated from pore-water samples.

e Temperature assumes a $40^{\circ} \mathrm{C} / 1000 \mathrm{~m}$ temperature gradient and a seafloor temperature of $2.9^{\circ} \mathrm{C}$.

the laboratory velocities where the hole is washed out. See Figure 1 in Boyce (this volume) which shows which velocities in Figures 19 and 20 are valid for a given hole diameter. These log-derived reflection coefficients agree, in general, with the major features of Sibuet's correlation of Hole 530A to the seismic profile in the site summary (this volume).

Figures 21 and 22 show reflection coefficients versus depth (from 0 to $1121 \mathrm{~m}$ ), which are derived using only the laboratory velocity-impedance data. The following discussions ignore requirements of the proper bed thicknesses for reflectors in a seismic profile.

Of course these show a greater number of potential reflectors than do the Sonic Log-derived reflection coefficients. This is because the Sonic Log data are a rolling average over a $1.0-\mathrm{m}$ interval $(0.5$ meter above and 0.5 meter below the calculated value), and because of the tool's movement up and down in the hole. The tool moves vertically depending on all movements of the D/V Glomar Challenger and the drill string.

Those reflectors in the upper $100 \mathrm{~m}$ of Hole 530A, in Holocene-Pleistocene diatom ooze and nannofossil ooze, are caused mainly by density variations, since sediment velocities are approximately the same as those of the interstitial seawater (excluding the debris-flow deposits). These density variations can be a function of (1) grain density, e.g., opal silica versus calcite, and (2) porosity variations. In Figures 4 and 5 , note that where densities are less than $1.52 \mathrm{~g} / \mathrm{cm}^{3}$ and porosities are greater than $71 \%$, the velocity is relatively constant and approximates that of the interstitial seawater. This zone in Figures 4 and 5 approximately represents the upper $100 \mathrm{~m}$ of the hole (disregarding debris-flow deposits). The data roughly follow the Wood (1941) equation, which has been shown to be approximately valid by many investigators (Shumway, 1960; Nafe and Drake,
1963; and others). Reflection coefficients versus depth in Figure 22 indicate that the mudstone from 277 to 467 $\mathrm{m}$ does not have very many reflectors; if they exist, they would be very weak. However, below $467 \mathrm{~m}$ the carbonate-cemented sandstones (limestone) and cemented mudstones create strong reflection coefficients.

The upper basalt contact at $1103 \mathrm{~m}$ does not appear to have significantly stronger reflection coefficients than do the carbonate coefficients above; however, its geometry (thick unit) would certainly be conducive to its being a significant reflector. Reflection cofficients of basalt below $1103 \mathrm{~m}$ are, however, very small; thus the seismic profiles here do not show reflectors below the upper contact of the basalt complex, unless there are interbedded lower velocity pillow basalts or sediments.

In Table 3 is the true formation resistivity, in a direction parallel to bedding, calculated from the Induction Log data, plus sound velocity (vertical) from the Sonic Log. These logs are from 625 to $945 \mathrm{~m}$ in Hole 530A. Table 3 also contains other associated parameters and data.

In Figure 23 is plotted velocity, from the Sonic Log, versus true (borehole corrected) formation electrical resistivity (parallel to bedding) from the Induction Log. The Velocity Log data are probably biased too low; thus this cross-plot does not show a valid relationship.

Figure 24 shows vertical laboratory velocity versus gravimetric porosity from 625 to $945 \mathrm{~m}$ in Hole 530A. From these data (Fig. 24) the following empirical relationship (Equation 3 ) is derived:

$$
\phi=\sqrt[0.527]{\frac{1.33}{V}}
$$

Equation 3 is used to calculate porosity from the Sonic Log for comparison with resistivity from the Induction 
Table 3. (Continued).

\begin{tabular}{|c|c|c|c|c|c|c|c|}
\hline \multirow{2}{*}{$\begin{array}{c}\text { GR } \\
\text { uncorrected } \\
\text { (sonic) } \\
\text { (API units) } \\
\end{array}$} & \multicolumn{2}{|c|}{ Velocity } & \multirow[t]{2}{*}{$\begin{array}{c}\phi \\
(\text { derived } \\
\text { from } V p)^{\mathrm{h}} \\
(\%)\end{array}$} & \multirow[t]{2}{*}{$\begin{array}{c}\text { Estimated } \\
\text { lithology } \\
\text { from GR logg } \\
(\%)\end{array}$} & \multirow{2}{*}{$\begin{array}{c}F \\
\left(R_{t} / R_{w}\right)\end{array}$} & \multirow{2}{*}{$=\sqrt[2]{\frac{1}{F}}$} & \multirow{2}{*}{ Lithology of cores } \\
\hline & $\mu \mathrm{s} / \mathrm{ft}$. & $\mathrm{km} / \mathrm{s}$ & & & & & \\
\hline 3 & 159.9 & 1.906 & 50.5 & 3.2 & 11.35 & 29.7 & Mudstone with limestone interbeds \\
\hline 8 & 139.5 & 2.185 & 39.0 & 19.3 & 14.06 & 26.7 & Mudstone with limestone interbeds \\
\hline 8 & 98.6 & 3.092 & 20.2 & 19.3 & 17.58 & 23.9 & Mudstone with limestone interbeds \\
\hline 8 & 176.6 & 1.726 & 61.0 & 19.3 & 8.84 & 33.6 & Mudstone with limestone interbeds \\
\hline 18 & 187.5 & 1.625 & 68.3 & 51.6 & 9.16 & 33.0 & Mudstone and marlstone \\
\hline 15 & 162.6 & 1.875 & 52.1 & 41.9 & 9.68 & 32.1 & Mudstone and marlstone \\
\hline 15 & 184.0 & 1.657 & 65.9 & 41.9 & 8.26 & 34.8 & Mudstone, marlstone and sandstone \\
\hline 17 & 154.9 & 1.968 & 47.5 & 48.4 & 10.34 & 31.1 & Mudstone, marlstone and sandstone \\
\hline 12 & 117.8 & 2.587 & 28.3 & 32.3 & 15.20 & 25.6 & Mudstone, marlstone, sandstone and limestone \\
\hline 18 & 177.9 & 1.713 & 61.9 & 51.6 & 7.89 & 35.6 & No recovery (probably as above) \\
\hline 16 & 126.7 & 2.406 & 32.5 & 45.2 & 18.52 & 23.2 & Siliclastic sandstone and mudstone \\
\hline 10 & 142.7 & 2.136 & 40.7 & 25.8 & 16.75 & 24.4 & Siliclastic sandstone \\
\hline 7 & 151.0 & 2.019 & 45.3 & 16.1 & 11.19 & 29.9 & Siliclastic sandstone \\
\hline 14 & 136.3 & 2.236 & 37.3 & 38.7 & 11.39 & 29.6 & Claystone \\
\hline 14 & 165.1 & 1.846 & 53.7 & 38.7 & 9.39 & 32.6 & Claystone \\
\hline
\end{tabular}

\footnotetext{
f In situ pore-water resistivity is extrapolated from U.S. Navy Hydrographic Office SP-11, 1956, and corrected for pressure using Techniques of Horne and Frysinger (1963): [Anonymous, 1956, Tables for Rapid Computation of Density and Electrical Conductivity of Sea Water, U.S. Navy Hydrographic Office, Special Publ. 11].

$\mathrm{g}$ GR (sonic) has a range of 2-33 API units; therefore estimate of percentage clay $=\frac{\mathrm{GR}-2}{33-2} \times 100$.

$\mathrm{h}_{\phi}=\sqrt[0.528]{\frac{1.33}{\mathrm{~V}}}$, where $\mathrm{V}=$ velocity in $\mathrm{km} / \mathrm{s}$.
}

Log in order to solve for an apparent interstitial water resistivity $\left(R_{w a}\right)$ curve versus depth. The Sonic Log had to be used for this purpose because the Density Log attempts were unsuccessful as a result of poor hole conditions.

Figure 25 shows the formation factor (from Table 3) versus porosity derived by using Equation 3 with velocity from the Sonic-Log data. Note that many of the $m$ values appear to be too high (greater than 2.6) relative to equations in Appendix A. These $m$ values may be artificially too high since they are based on biased data. The bias probably results from the fact that the velocity obtained from the Sonic Log is too low; thus the derived porosity (Equation 3) is too high for a given true formation resistivity value.

The $R_{w a}$ curve (Fig. 26) is calculated by rearranging the Archie (1942) equation: $R_{w a}=$ (Resistivity Induction $\log )\left(\phi^{2}\right)$, where $\phi$ is the fractional-porosity derived from Sonic Log (Equation 3). $R_{w a}$ is plotted versus depth and is used here mainly as a tool to identify zones of: (1) metallic mineral deposits, (2) temperature anomalies, (3) interstitial-water salinity anomalies, and (4) hydrocarbons. It is not designed to calculate $R_{w a}$ accurately (borehole corrections are not applied), but only to indicate the presence of anomalous lithologic zones.

In Figure 26, there are no large anomalies (unfortunately, the logging data are above the black shale beds), and the variations seen are noise in the data: (1) slight misalignment of the depths of the velocity and Induction Log data; (2) thin beds with contrasting resistivities, since the Induction Log resistivities were not adjusted for borehole conditions; and (3) the $1.2-\mathrm{m}$ resolution of the Induction Log relative to the $61-\mathrm{cm}$ vertical resolution of the Velocity Log. Theoretically the $R_{w a}$ plot should decrease slightly with increasing depth because of increasing temperature.

Vane shear strength versus depth is shown in Figures 27 and 28 (coded for lithology) for Sites 530 and 532 (Holes 532 and 532B). Many of these samples, particularly those below $130 \mathrm{~m}$ at Site 532, are gassy; thus the vane shear strength may be less than that of comparable sediments which are water saturated, and partially the result of disturbance of sediment as gas expands (Dover et al., 1981). Vane shear strength of gassy samples may not represent in situ conditions, for the sediments may not contain gas at in situ depths. From 0 to $100 \mathrm{~m}$, vane shear strength uniformly increases from about $80 \mathrm{~g} / \mathrm{cm}^{2}$ to about $800 \mathrm{~g} / \mathrm{cm}^{2}$. From about 100 to about $300 \mathrm{~m}$, vane shear strength varies irregularly with increasing depth, ranging from about 500 to $2300 \mathrm{~g} / \mathrm{cm}^{2}$. At Site 532 , vane shear strength actually decreases slightly with increasing depth (disturbance of sediment by expanding gas), which agrees with similar data at Site 362 (Bolli and Ryan et al., 1978). Vane shear strength at Site 362 tended to be significantly less than at Site 532, probably as a result of the disturbance of Site 362 cores by rotary coring methods; it is also possible that these sediments are significantly different from those at Site 532 .

In Figures 29, 30, and 31 are presented vane shear strength versus gravimetric porosity, gravimetric wetbulk density, and horizontal sound velocity. These are coded for lithology. In these plots some grouping does occur; however, this probably results, in part, from a limited number of data for each lithologic type-e.g., siliceous ooze in Figures 29 and 31-which relate vane shear strength to porosity and velocity. In the vane 


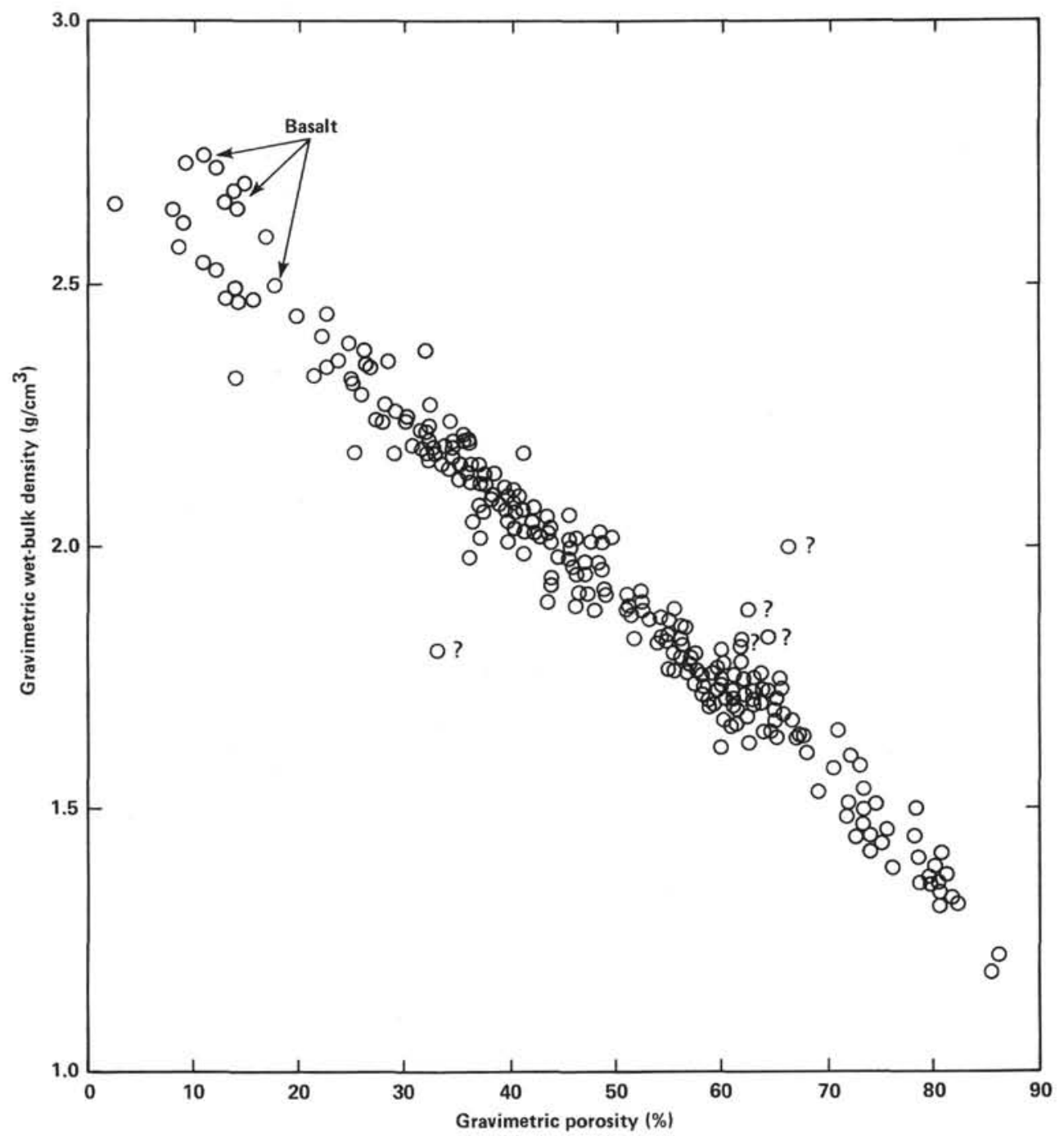

Figure 2. Gravimetrically determined wet-bulk density versus gravimetrically determined porosity, Sites 530 and 532.

strength-density plot (Fig. 30), the siliceous sediments are distinctly set apart from the other data; this is in part caused by its lower grain density of opaline silica.

The high-porosity siliceous (diatoms) sediment appears to have distinctly higher values for vane shear strength for a given porosity than do other sediment types. This is partially related to the structural strength of the framework of the diatom fossils (Hamilton, 1976).

\section{SUMMARY AND CONCLUSIONS}

1. At Site 530 , from 0 to $100 \mathrm{~m}$ below the seafloor in Holocene to Pleistocene diatom and nannofossil ooze (excluding debris-flow deposits), the sound velocity of undisturbed samples is approximately equivalent to that of the interstitial water; thus, reflectors are caused by grain density changes (e.g., opal silica to calcite) and porosity changes, and not significantly by velocity variations. These low velocities are in theoretical agreement with Wood's (1941) equation.

2. Reflection coefficients derived from laboratory data agree in general (at least in the upper part of Hole $530 \mathrm{~A}$ ) with the major features of the seismic profile (see site summary, this volume). It suggests more potential reflectors than indicated by the reflection coefficients derived from the Gearhart-Owen sonic log from 625 to $940 \mathrm{~m}$ (since the sonic log data average thin beds).

3. From 0 to $467 \mathrm{~m}$, at laboratory temperature and pressures, velocities are 1.5 to $1.8 \mathrm{~km} / \mathrm{s}$; below $200 \mathrm{~m}$ these increase irregularly with increasing depth. From 0 to $100 \mathrm{~m}$ in Holocene to Pleistocene nannofossil and diatom ooze, velocities are approximately equivalent to that of the interstitial seawater. From 100 to $467 \mathrm{~m}$, in Pliocene-Oligocene nannofossil ooze, clay, marl, mudstone, and debris flows, acoustic anisotropy irregularly increases to $10 \%$, with 2 to $5 \%$ being typical. From 467 to $1103 \mathrm{~m}$, in Eocene to late Albian-early Cenomanian interbedded mudstone, marlstone, chalk, clastic limestone, sandstone, and black shale, velocities range from 1.6 to $5.48 \mathrm{~km} / \mathrm{s}$, and acoustic anisotropies are as great as $47 \%(1.0 \mathrm{~km} / \mathrm{s})$ faster horizontally. Mudstone and uncemented sandstone have anisotropies which irregularly increase with increasing depth from 5 to $10 \%(0.2$ $\mathrm{km} / \mathrm{s}$ ). Calcareous mudstone has the greatest anisotropies, typically $35 \%(0.6 \mathrm{~km} / \mathrm{s})$. 


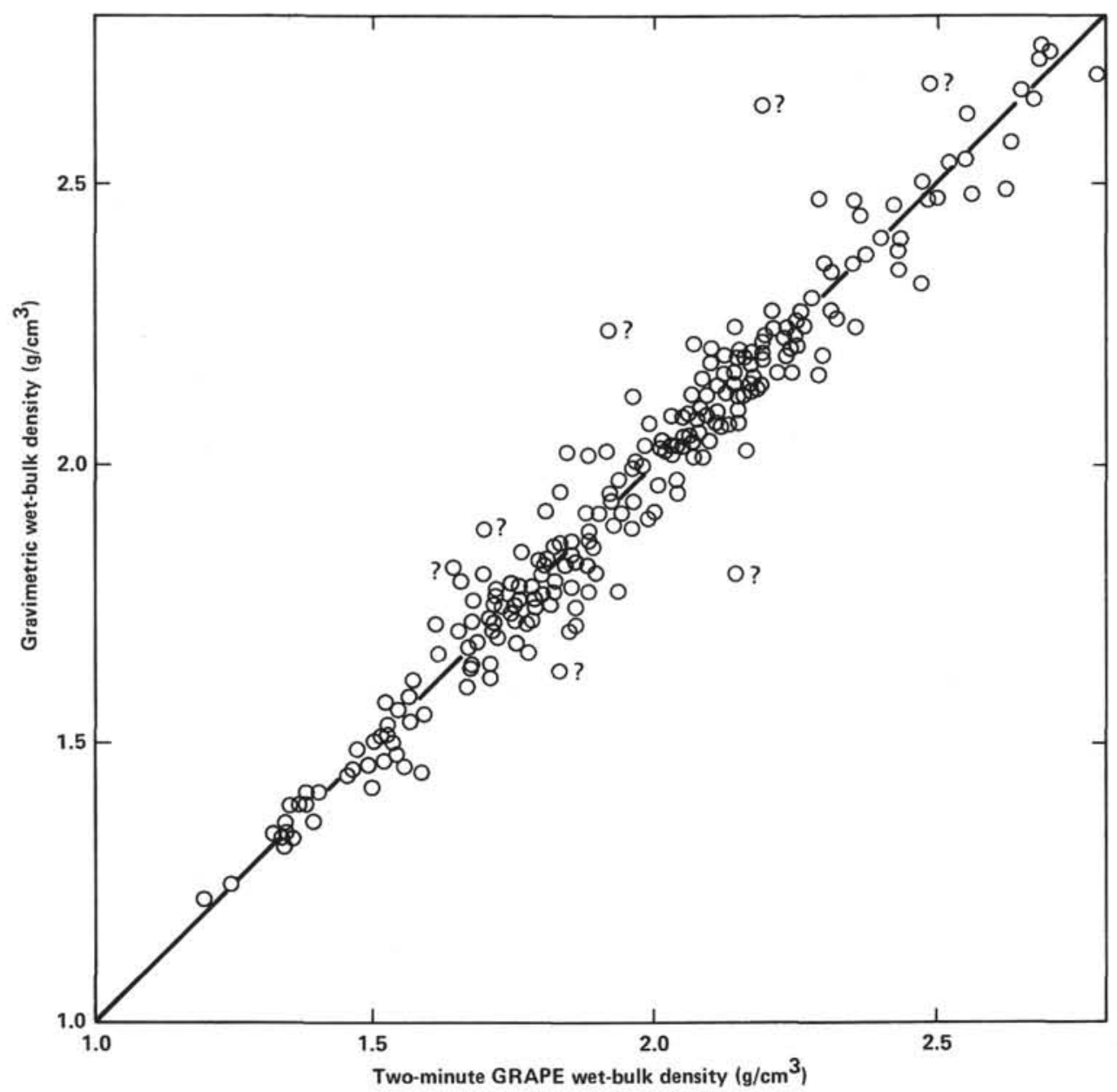

Figure 3. Gravimetrically determined wet-bulk density versus two-minute GRAPE-determined wet-bulk density, Sites 530 and 532.

4. In situ velocities are calculated for the laboratorymeasured data and are corrected for in situ temperature, hydrostatic pressure, and porosity rebound (expansion when the overburden pressure is released); however, they are not corrected for rigidity changes related to overburden pressure. These corrections affect the semiconsolidated sedimentary rocks most (up to $0.25 \mathrm{~km} / \mathrm{s}$ faster). Sonic Log velocities appeared to be less than laboratory data.

5. Measurements of porosity-density versus depth for mud, mudstone, and pelagic oozes agree with those for similar sediments in Hamilton's (1976) summary. In the area of about $400 \mathrm{~m}$ and about $850 \mathrm{~m}$ are zones of relatively higher porosity for mudstone, which may suggest overpressurized pore water; however, they are more likely to be caused by variations in grain size distribution and lithology.

6. Electrical resistivity, in a direction parallel to bedding, from 625 to $950 \mathrm{~m}$, ranged from about 1.0 to 4.0 ohm-m in Maestrichtian to Santonian-Coniacian interbedded mudstone, marlstone, chalk, clastic limestone, and sandstone. An interstitial water resistivity curve did not indicate any unexpected lithology or unusual fluids or gases in the pores of the rocks. These logs were above the black shale beds.
7. From 0 to $100 \mathrm{~m}$ at Sites 530 and 532 , the vane shear strength on undisturbed samples of HolocenePleistocene diatom and nannofossil oozes uniformly increases from about $80 \mathrm{~g} / \mathrm{cm}^{2}$ to about $800 \mathrm{~g} / \mathrm{cm}^{2}$. From 100 to $300 \mathrm{~m}$, vane shear strength of Pleistocene-Miocene nannofossil ooze, clay, and marl is irregular versus depth with a range of 500 to $2300 \mathrm{~g} / \mathrm{cm}^{2}$; at Site 532 the vane shear strength appears to decrease irregularly and slightly with increasing depth (gassy zone); this is probably an artifact of disturbed sediments caused by expanding gas at atmospheric pressure. Because these sediments may not be gassy at in situ depths; the values on gassy samples below $130 \mathrm{~m}$ at Site 532 are probably not representative of in situ values.

\section{ACKNOWLEDGMENTS}

The author wishes to thank Dr. E. L. Hamilton and Mr. A. H. Jageler for reviewing the manuscript. Mr. Tom Birtley developed and ran all requested computer plots.

\section{REFERENCES}

Archie, G. E., 1942. The electrical resistivity log as an aid in determining some reservoir characteristics. Trans. Am. Inst. Min. Metall. Pet. Eng., 146:54-62.

1947. Electrical resistivity an aid in core analysis interpretation. Am. Assoc. Petrol. Geol. Bull., 31:350. 
ASTM, 1975. Standard method for field vane shear test in cohesive soil: ASTM Designation: D 2473-72. 1975 Annual Book of ASTM Standards, Pt. 19. Natural Building Stones: Soil and Rock, Peats, Mosses, and Humus: Philadelphia (ASTM), pp. 221-324.

Bachman, R. T., 1979. Acoustic anisotropy in marine sediments and sedimentary rocks. J. Geophys. Res., 84(1113):7467-7663.

Bedcher, A. Z., 1965. Electrical anisotropy of clays: Its geological significance. Int. Geol. Rev., 7:7-10.

Berg, J. W., Jr., 1952. Conductivity study of aqueous kaolin $\mathrm{NaC1}$ mixtures. Prod. Mon., 16:36-44.

Birch, F., 1961. The velocity of compressional waves in rocks to 10 kilobars, Pt. 2. Geophys. Res., 66:2199.

Bolli, H. M., Ryan, W. B. F., and Shipboard Scientific Party, 1978. Walvis Ridge Sites 362 and 363. Init. Repts. DSDP, 40: Washington (U.S. Govt. Printing Office), 183-356.

Boyce, R. E., 1968. Electrical resistivity of modern marine sediments from the Bering Sea. J. Geophys. Res., 73:4759-4766.

1976a. Definitions and laboratory techniques of compressional sound velocity parameters and wet-water content, wet-bulk density, and porosity parameters by gravimetric and gamma-ray attenuation techniques. In Schlanger, S. O., Jackson, E. D., et al., Init. Repts. DSDP, 33: Washington (U.S. Govt. Printing Office), 931-958.

1976b. Sound velocity-density parameters of sediment and rock from DSDP drill Sites 315-318 on the Line Islands Chain, Manihiki Plateau and Tuamotu Ridge in the Pacific Ocean. In Schlanger, S. O., Jackson, E. D., et al., Init. Repts. DSDP, 33: Washington (U.S. Govt. Printing Office), 695-728.

1977. Deep Sea Drilling Project procedures for shear strength measurement of clayey sediment using a modified Wykeham Farrance laboratory vane apparatus. In Barker, P. F., Dalziel, I. W. D., et al., Init. Repts. DSDP, 36: Washington (U.S. Govt. Printing Office), 1059-1072.

1980. Determination of the relationships of electrical resistivity, sound velocity, and density/porosity of sediment and rock by laboratory techniques and well logs from Deep Sea Drilling Project Sites 415 and 416 off the coast of Morocco. In Lancelot, Y., Winterer, E. L., et al., Init. Repts. DSDP, 50: Washington (U.S. Govt. Printing Office), 305-318.

1981. Electrical resistivity, sound velocity, thermal conductivity, density-porosity, and temperature, obtained by laboratory techniques and well logs: Site $\mathbf{4 6 2}$ in the Nauru Basin of the Pacific Ocean. In Larson, R. E., Schlanger, S. O., et al., Init. Repts. DSDP, 61: Washington (U.S. Govt. Printing Office).

Bullen, K. E., 1947. Introduction to the Theory of Seismology: London (Cambridge University Press).

Carlson, R. L., and Christensen, N. I., 1977. Velocity anisotropy and physical properties of deep-sea sediments from the western South Atlantic. In Supko, P. R., Perch-Nielsen, K., et al., Init. Repts. $D S D P, 39$ : Washington (U.S. Govt. Printing Office), 555-559.

Christensen, N. I., Hyndman, R. D., Hall, J. M., and Salisbury, M. H., 1979. Seismic velocities, densities, and porosities of basalt from DSDP Leg 46. In Dmitriev, L., Heirtzler, J., et al., Init. Repts. DSDP, 46: Washington (U.S. Govt. Printing Office), 383-388.

Christensen, N. I., and Salisbury, M. H., 1975. Structure and constitution of the lower oceanic crust. Rev. Geophys. Space Phys., 13: 57-86.

Coulomb, C. A., 1776. Essai sur une application des regles de maximis et minimis à quelques problemes de statique, relatifs à l'architecture. Mem. Mathematique Phys., L'Academie Roy. Sci. (Paris), pp. 343-382.

de Witte, L., 1950a. Resistivity and saturation distribution in infiltrated zones of porous formations around drill holes. Oil \& Gas J., 49:246-268.

1950b. Relations between resistivities and fluid contents of porous media. Oil \& Gas J., 49:120-132.

Donnelly, T., Francheteau, J. Bryan, W., Robinson, P., Flower, M., Salisbury, M., et al., 1980. Init. Repts. DSDP, 51, 52, 53: Washington (U.S. Govt. Printing Office).

Dover, A. R., Audbert, J. M. E., and Bea, A. G., 1981. Quality in soil borings makes a difference. Oil \& Gas J., 79(26):133-139.

Gregory, A. R., 1977. Aspects of rock physics from laboratory and log data that are important to seismic interpretation. In Payton, C. H. (Ed.), Seismic Stratigraphy-Applications to Hydrocarbon Exploration, AAPG Mem., 36:15-46.
Hamano, Y., 1980. Physical properties of basalts from Holes 417D and 418A. In Donnelly, T., Francheteau, J., Bryan, W., Robinson, P., Flower, M., Salisbury, M., et al., Init. Repts. DSDP, 51, 52, 53, Pt. 2: Washington (U.S. Govt. Printing Office), 1957-1966.

Hamilton, E. L., 1965. Sound speed and related physical properties of sediments from experimental Mohole (Guadalupe Site): Geophysics, 30:257-261. 1971. Elastic properties of marine sediments. J. Geophys. Res., 76:579-604.

1976. Variations of density and porosity with depth in deep-sea sediments. J. Sediment. Petrol., 46(2):280-300. 1978. Sound velocity-density relations in sea floor sediments and rocks. J. Acoust. Soc. Am., 63(2):366-377.

1980. Geoacoustic modeling of the seafloor. J. Acoust. Soc. Am., 68:1313-1340.

Hamilton, E. L., and Menard, H. W., 1956. Density and porosity of sea floor surface sediments off San Diego, California. $A A P G$ Bull., 40(4):754-761.

Horn, D. R., Delach, M. N., and Horn, B. M., 1969. Physical properties of sedimentary provinces, North Pacific and North Atlantic Oceans. In Inderbitzen, A. L. (Ed.), Deep Sea Sediments: New York (Plenum), pp. 417-441.

Horne, R. A., 1965. The physical chemistry and structure of sea water. Water Resour. Res., Second Quarter, 1:263-276.

Horne, R. A., and Courant, R. A., 1964. Application of Walden's rule to the electrical conduction of sea water. J. Geophys. Res., 69:1971-1977.

Horne, R. A., and Frysinger, G. R., 1963. The effect of pressure on the electrical conductivity of sea water. J. Geophys. Res., 68: 1967-1973.

Howell, B. F., Jr., 1953. Electrical conduction in fluid saturated rocks. World Oil, 136(Pt. I):113-116.

Hvorslev, M. J., 1936. Conditions of failure for remolded cohesive soils. First Intern. Conf. Soil Mech. Found. Eng., 3:51-53.

1937. Uber die festigkeitseignenschaften gestorter bindiger boden: Ingeniorridenskabelige Skriftor A, Danmark, Naturvid, Samfund. Copenhagen.

Hyndman, R. D., 1977. Seismic velocity measurements of basement rocks from DSDP Leg 37. In Aumento, F., Melson, W. G., et al., Init. Repts. DSDP, 37: Washington (U.S. Govt. Printing Office), 373-387.

Jones, L. E. A., and Wang, H. F., 1981. Ultrasonic velocities in Cretaceous shales from the Williston Basin. Geophysics, 46(3):288-297.

Keller, G. V., 1951. The role of clays in the electrical conductivity of the Bradford Sand. Prod. Mon., 15:23-28.

1966. Electrical properties of rock and minerals. In Clark, S. P. (Ed.), Handbook of Physical Constants. Geol. Soc. Am. Mem., 97:553-580.

Keller, G. H., and Bennett, R. H., 1973. Sediment mass physical properties-Panama Basin and Northeastern Equatorial Pacific. In van Andel, Tj. H., Heath, G. R., et al., Init. Repts. DSDP, 16: Washington (U.S. Govt. Printing Office), 494-512.

Keller, G. V., and Frischknecht, F. C., 1966. Electrical Methods in Geophysical Prospecting: New York (Pergamon Press).

Kermabon, A., Gehin, C., and Blavier, P., 1969. A deep-sea electrical resistivity probe for measuring porosity and density of unconsolidated sediment. Geophysics, 34:554-591.

Kravitz, F. H., 1970. Repeatability of three instruments used to determine the undrained shear strength of extremely weak, saturated, cohesive sediments. J. Sediment. Petrol., 40:1026-1037.

Lambe, T. W., 1960. A mechanistic picture of shear strength in clay. Am. Soc. Civil Eng., Res. Conf. on Shear Strength of Cohesive Soils (Boulder, Colorado, June 1960), pp. 555-580.

Lambe, T. W., and Whitman, R. V., 1969. Soil Mechanics: New York (John Wiley and Sons, Inc.).

Laughton, A. S., 1957. Sound propagation in compacted ocean sediments. Geophysics, 22:233-260.

Lee, H. J., 1973. Measurements and estimates of engineering and other physical properties, Leg 19. In Creager, J. S., Scholl, D. W. et al., Init. Repts. DSDP, 19: Washington (U.S. Govt. Printing Office), 701-720.

Matthews, D., 1979. Shipboard measurements of seismic velocity, density, and porosity in DSDP Leg 46 basalts. In Dmitriev, L., Heirtzler, J., et al., Init. Repts. DSDP, 46: Washington (U.S. Govt. Printing Office), 379-382. 
Maxwell, J. C., 1904. Electricity and Magnetism (Vol. 1): Oxford (Clarendon Press).

Moore, D. G., 1964. Shear strength and related properties of sediments from experimental mohole (Guadalupe Site): J. Geophys. Res., 69:4271-4291.

Nafe, J. E., 1963. Physical properties of marine sediments. In Hill, M. N. (Ed.), The Sea (Vol. 3): New York (Interscience), pp. 749-815.

Nafe, J. E., and Drake, C. L., 1957. Variation with depth in shallow and deep water marine sediments of porosity density and the velocities of compressional and shear waves. Geophysics, 22:523-552.

Patnode, H. W., and Wyllie, M. R. J., 1950. The presence of conductive solids in reservoir rocks as a factor in Electric Log interpretation. J. Pet. Technol., 2:42-52.

Press, F., 1966. Seismic velocities. In Clark, S. P. (Ed.), Handbook of Physical Constants. Mem. Geol. Soc. Am., 97:195.

Richards, A. F., 1961. Investigations of deep sea sediment cores, Pt. I: shear strength, bearing capacity, and consolidation. Washington (U.S. Navy Hydrographic Office), Tech. Rept. 63.

Rocker, K., 1974. Vane shear strength measurements on Leg 27 sediment. In Veevers, J. J., Heirtzler, J. R., et al., Init. Repts. DSDP, 27: Washington (U.S. Govt. Printing Office), 425-432.

Salisbury, M. H., Donnelly, T. W., and Francheteau, J., 1980. Geophysical logging in Deep Sea Drilling Project Hole 417D. In Donnelly, T., Francheteau, J., Bryan, W., Robinson, P., Flower, M., Salisbury, M., et al., Init. Repts. DSDP, 51, 52, 53, Pt. 1: Washington (U.S. Govt. Printing Office), 705-714.

Schlumberger Ltd., 1972. Log Interpretation Charts: New York (Schlumberger, Ltd.).

Schmertmann, J. H., and Osterberg, J. O., 1960. An experimental study of the development of cohesion and friction with axial strain in saturated cohesive soils. Am. Soc. Civil Eng., Res. Conf. on Shear Strength of Cohesive Soils (Boulder, Co., June 1980), pp. 643-694.

Scholl, D. W., 1963. Techniques for removing interstitial water from coarse-grained sediments for chemical analyses. Sedimentology, 2: 156-163.

Scott, R. F., and Schoustra, J. J., 1968. Soil Mechanics and Engineering: New York (McGraw-Hill)

Shumway, G., 1960. Sound speed and absorption studies of marine sediments by a resonance method. Geophysics, 25:451-467.

Siever, R., Garrels, R. M., Kanwisher, J., and Berner, R. A., 1961. Interstitial waters of Recent marine muds off Cape Bod. Science, 134:1071-1072.

Siever, R., Kevin, C. B., and Berner, R. A., 1965. Composition of interstitial waters of modern sediments. J. Geol., 73:39-73.

Thomas, B. D., Thompson, T. G., and Utterback, C. L., 1934. The electrical conductivity of sea water. Conseil, Internat. Explor. Mer., 9:28-35.

Winsauer, W. O., and McCardell, W. M., 1953. Ionic double-layer conductivity in reservoir rock. Trans. Am. Inst. Min. Metall. Pet. Eng., 198:129-134.

Winsauer, W. O., Shearin, H. M., Jr., Masson, P. H., et al., 1952. Resistivity of brine-saturated sands in relation to pore geometry. Am. Assoc. Pet. Geol. Bull., 36:253-277.

Wood, A. B., 1941. A Textbook of Sound: New York (MacMillan). Wu, T. H., 1966. Soil mechanics. Boston (Allyn and Bacon).

Yong, R. N., and Warkentin, B. P., 1966. Introduction to Soil Behavior. New York (Macmillan).

Wyllie, M. R. J., 1955. Role of clay in well-log interpretation on clays and clay technology. Bull. Calif. Div. Mines Geol., 169:282-305.

Wyllie, M. R. J., and Gregory, A. R., 1953. Formation factors of unconsolidated porous media: Influence of particle shape and effect of cementation. J. Pet. Technol., 198:103-110.

Wyllie, M. R. J., Gregory, H. R., and Gardner, L. W., 1956. Elastic waves in heterogeneous and porous media. Geophysics, 21:41-70.

Wyllie, M. R. J., and Southwick, P. F., 1954. An experimental investigation of the S.P. and resistivity phenomena in dirty sands. $J$. Pet. Technol., 6:44-57.

Date of Initial Receipt: August 31, 1981

\section{APPENDIX A \\ Electrical Formation Factor-Porosity Relations of Wet-Saturated Sedimentary Rock and Sediment ${ }^{1}$}

Maxwell (1904) theoretically derived the following relationship for a suspension of spheres:

$$
F=\frac{3-\phi}{2 \phi}
$$

where $F=R_{o} / R_{w}=$ formation factor; $R_{o}=$ the electrical resistivity of the saturated formation; $R_{w}=$ the resistivity of the interstitial water; and $\phi=$ the porosity expressed as a fraction or decimal.

Archie's (1942) equation was derived for consolidated sandstone without clay material:

$$
F=\phi^{-m}=\phi^{-2}
$$

where $m$ is a variable depending on consolidation, textures, and cementation.

Winsauer et al. (1952) derived a slightly different empirical formula for various sandstone formations:

$$
\mathrm{F}=a \phi^{-m}=0.62 \phi^{-2.15}
$$

where $a$ and $m$ are variables depending on cementation, textures, and mineralogy of the formation.

Boyce (1968) derived the following empirical equation for modern marine diatomaceous silty clay to silty sand (this equation is of the same form as that of Winsauer et al., 1952):

$$
F=1.30 \phi^{-1.45}
$$

Kermabon et al. (1969) derived the following empirical equation (one of three), also for modern marine clays and turbidite sands:

$$
F=\left(\frac{1.45}{\phi}\right)^{1.46}-0.719 \text {. }
$$

\section{APPENDIX B}

Theoretical Equations Relating Compressional Velocity of the Wet-Saturated Rock to the Velocities and Densities of the Fluid and Solid Grain-End-Member Constituents

The Wood (1941) equation applies to velocities through suspensions without rigidity:

$$
V_{b}=\left(\frac{1}{\left[\phi \beta_{w}+(1-\phi) \beta_{g}\right)\left(\phi \varrho_{w}+(1-\phi) \varrho_{g}\right]}\right)^{1 / 2}
$$

where $V=$ compressional velocity; $\varrho=$ density, $\beta=$ compressibility, and subscripts $g, w$, and $b$ represent the solid grains, intersititial water, and wet-bulk rock or sediment, respectively; and $\phi=$ fractional porosity, where $\varrho_{b}=\phi \varrho_{w}+(1-\phi) \varrho_{g}$.

The Wyllie et al. (1956) equation applies in rocks with rigidity:

$$
\frac{1}{V_{b}}=\frac{\phi}{V_{w}}+\frac{(1-\phi)}{V_{g}} .
$$

The Nafe and Drake (1957) equation applies to rock with varying degrees of ridigity, which is controlled in the equation by the value of $n$ :

$$
V_{b}^{2}=\phi V_{w}^{2}\left[1+\left(\frac{\varrho_{w}}{\varrho_{b}}\right)(1-\phi)\right]+\frac{\varrho_{g}}{\varrho_{b}}(1-\phi)^{n} V_{g}^{2}
$$

\footnotetext{
${ }^{1}$ Keller (1966) and Keller and Frischknecht (1966) summarize and discuss similar equations derived for continental formations.
} 


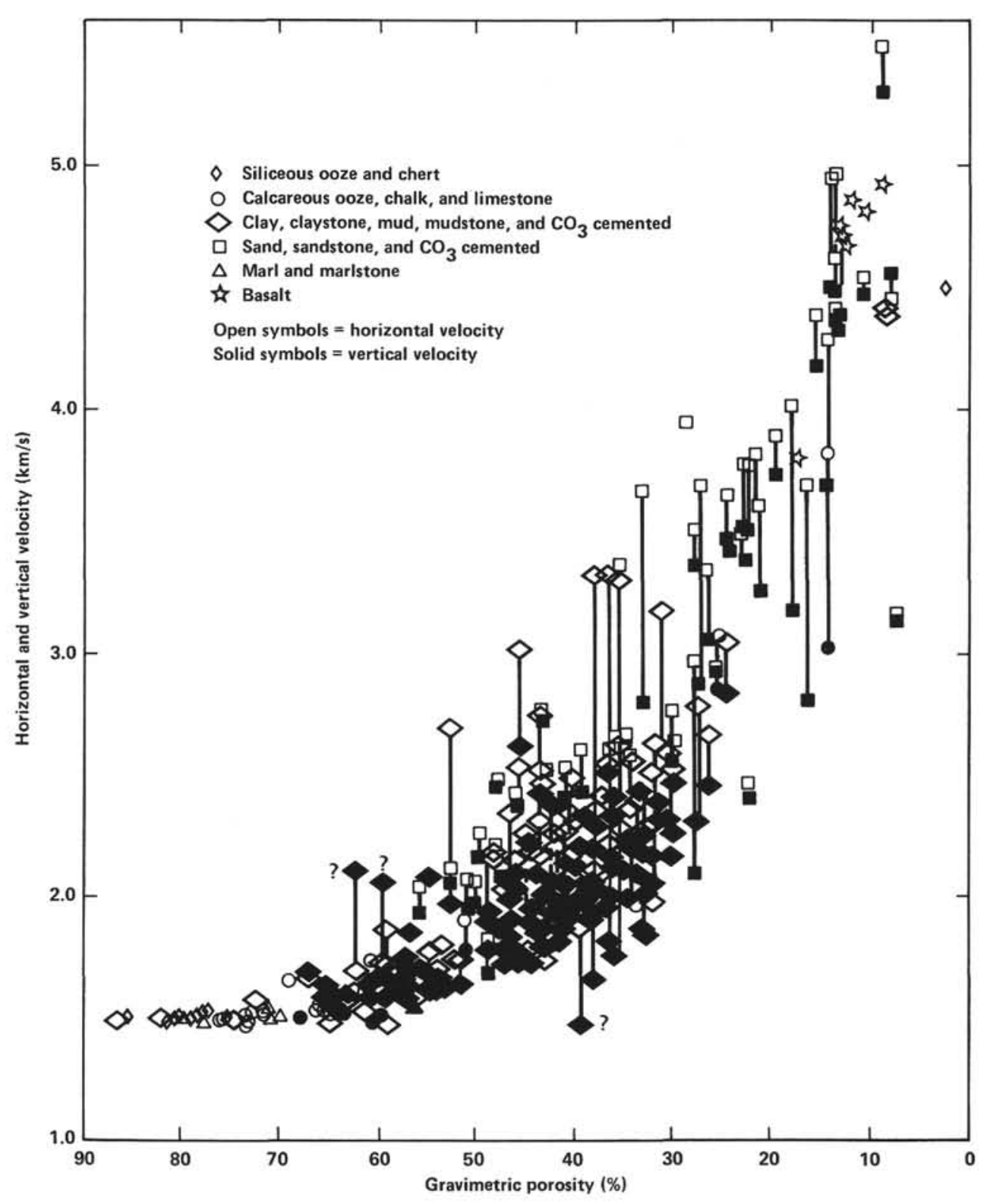

Figure 4. Laboratory horizontal and vertical velocity versus gravimetrically determined porosity, Sites 530 and 532 . 


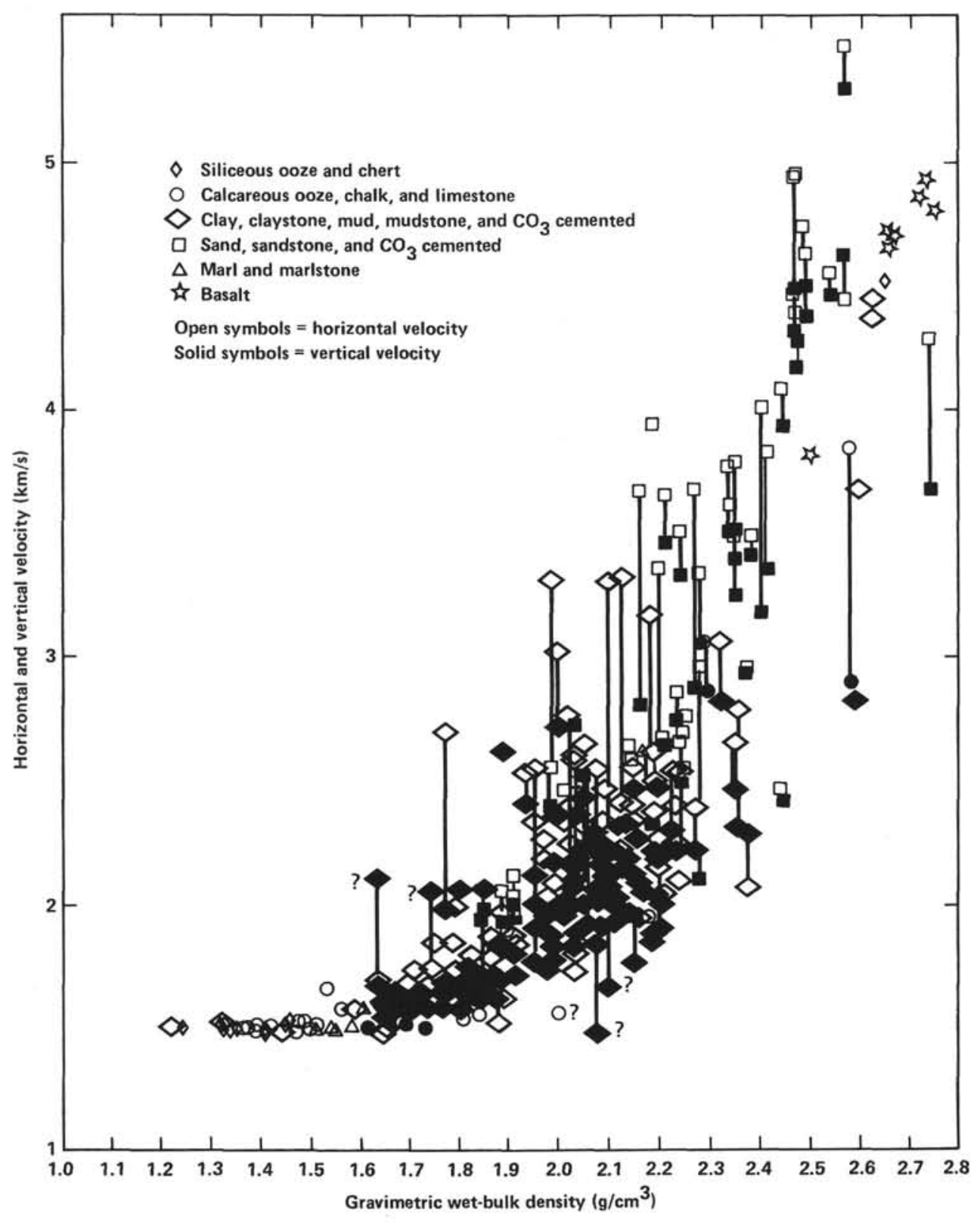

Figure 5. Laboratory horizontal and vertical velocity versus gravimetrically determined wet-bulk density, Sites 530 and 532. 


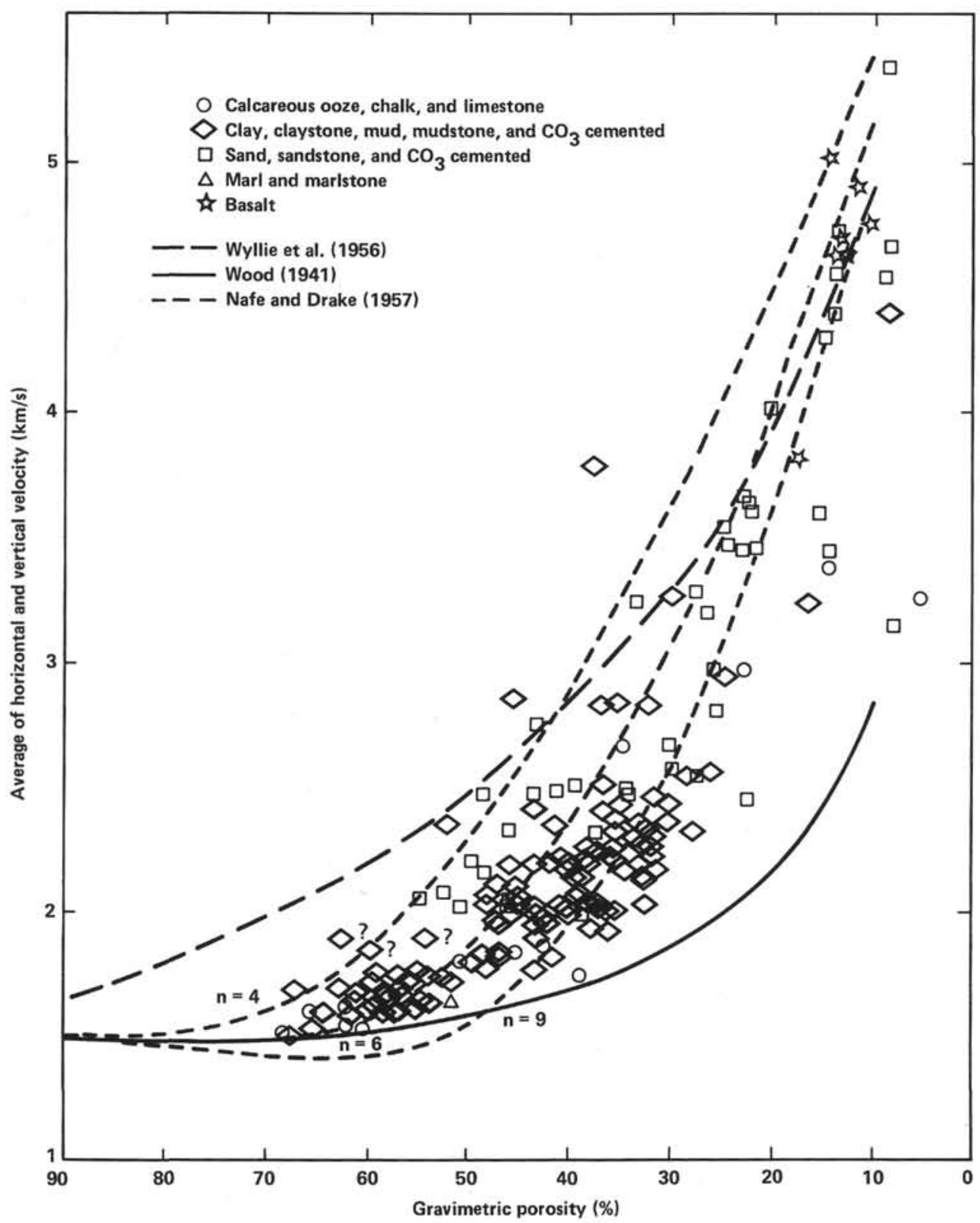

Figure 6. Average of laboratory horizontal and vertical velocity versus gravimetrically determined porosity, Hole 530A. Included are equations of Wood (1941), Wyllie et al. (1956), and Nafe and Drake (1957), assuming a limestone matrix $\left(2.72 \mathrm{~g} / \mathrm{cm}^{3}, 6.45 \mathrm{~km} / \mathrm{s}\right)$ with seawater $\left(1.025 \mathrm{~g} / \mathrm{cm}^{3}, 1.53 \mathrm{~km} / \mathrm{s}\right)$ in the pores. 


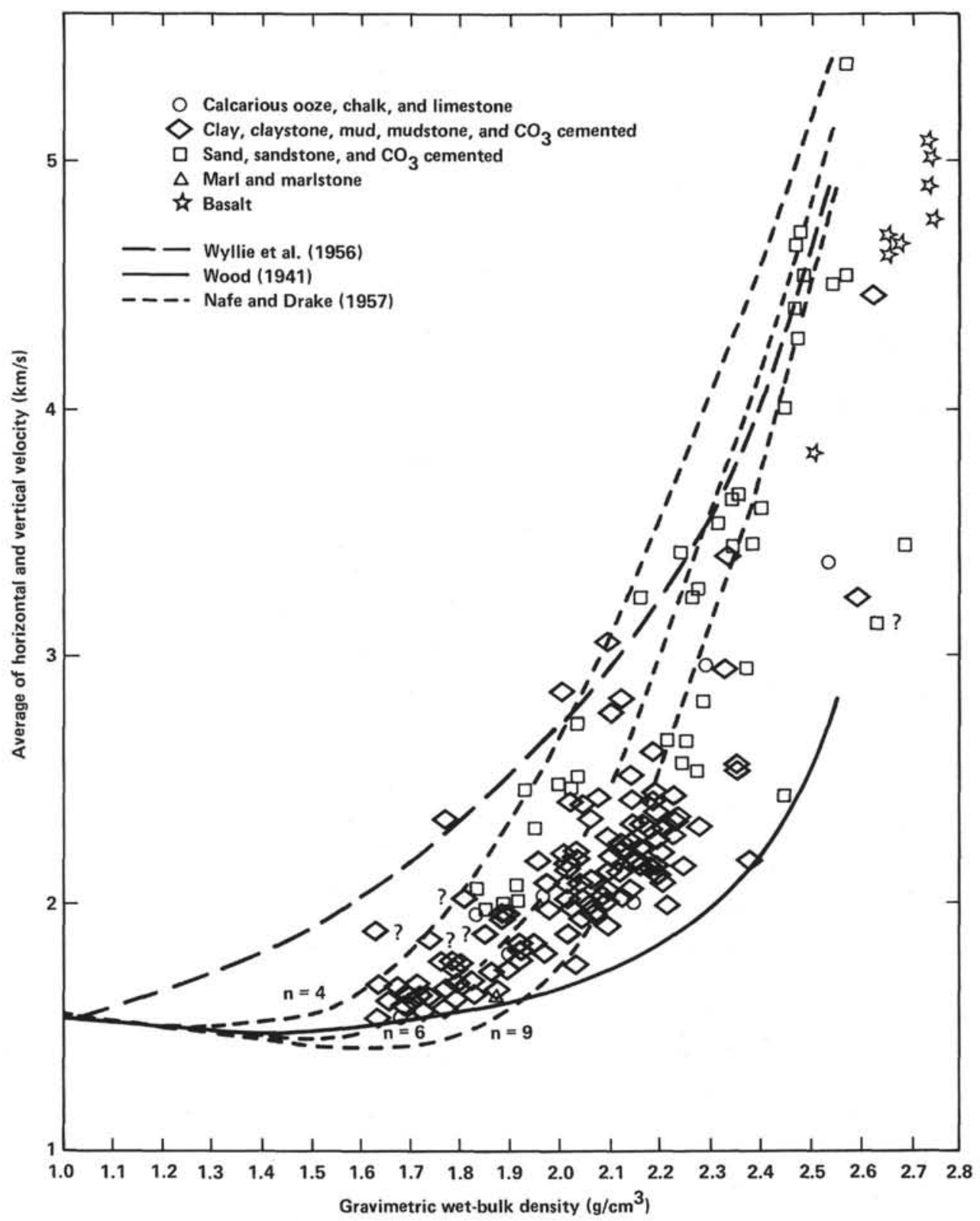

Figure 7. Average of laboratory horizontal and vertical velocity versus gravimetrically determined wetbulk density, Hole 530A. Included are equations of Wood (1941), Wyllie et al. (1956), and Nafe and Drake (1957), assuming a limestone matrix $\left(2.72 \mathrm{~g} / \mathrm{cm}^{3}, 6.45 \mathrm{~km} / \mathrm{s}\right)$ with seawater $\left(1.025 \mathrm{~g} / \mathrm{cm}^{3}\right.$, $1.53 \mathrm{~km} / \mathrm{s}$ ) in the pores. 


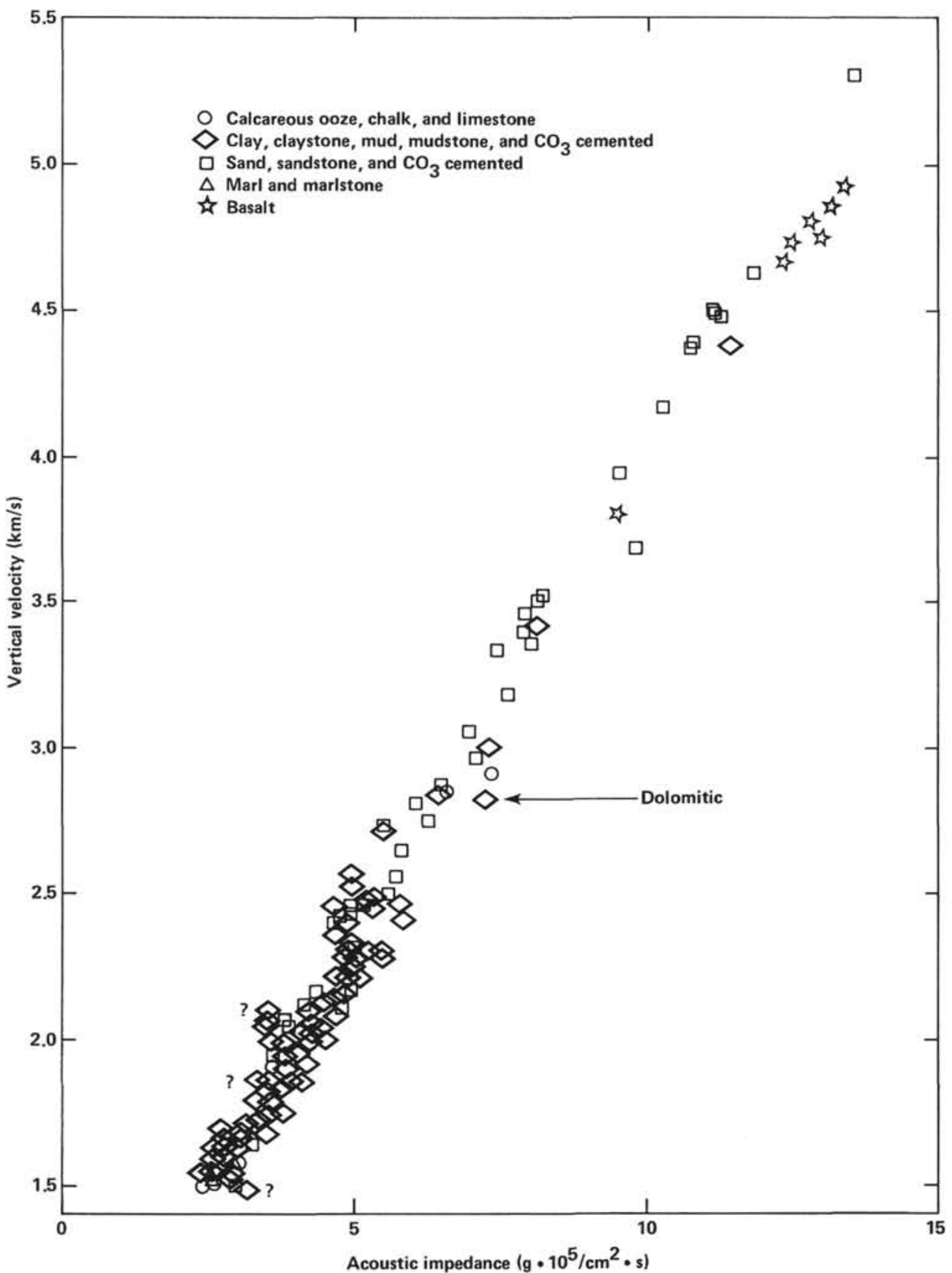

Figure 8. Laboratory determined vertical velocity versus impedance, Hole 530A. 


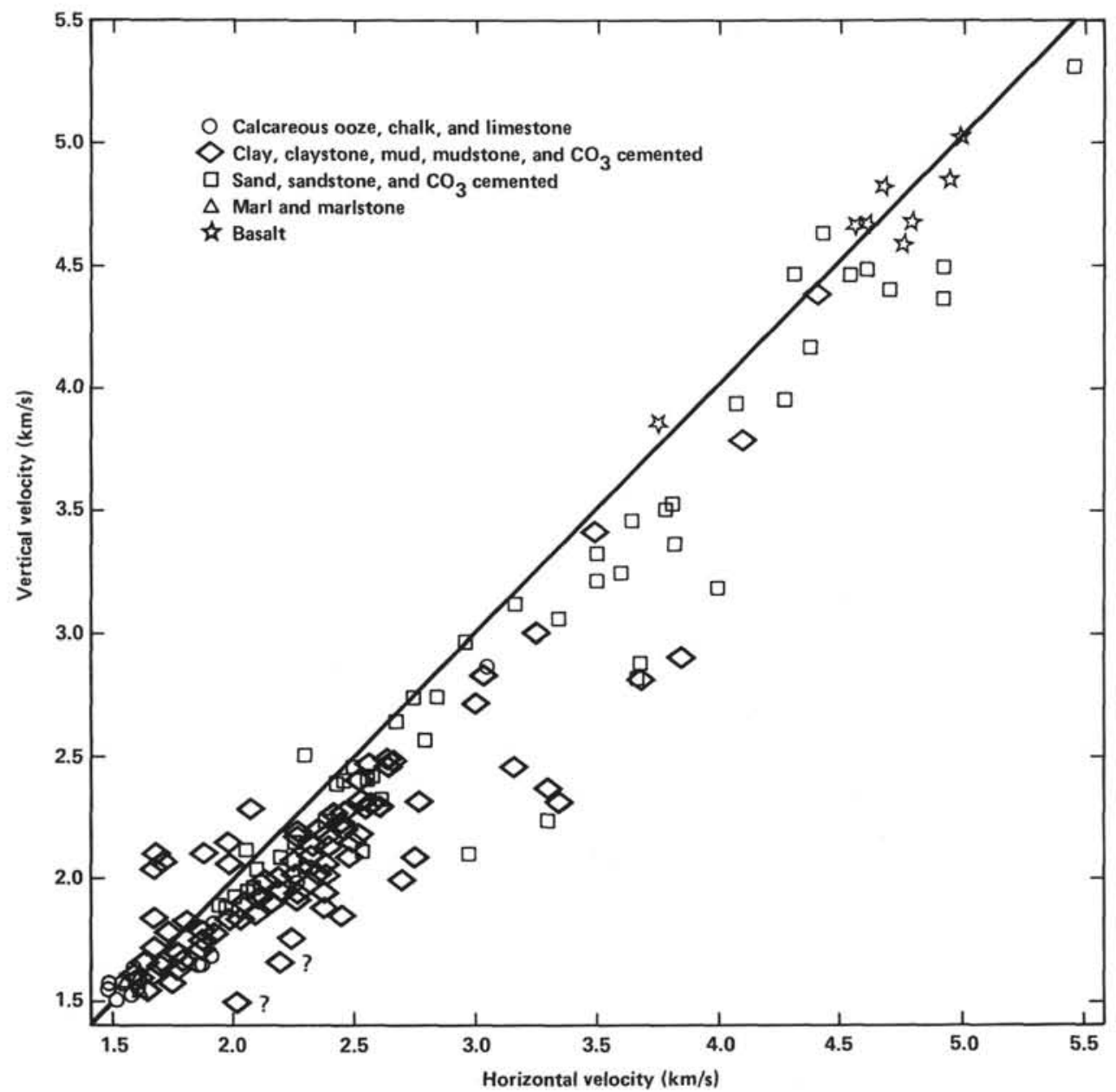

Figure 9. Laboratory vertical velocity versus laboratory horizontal velocity, Hole 530A. 


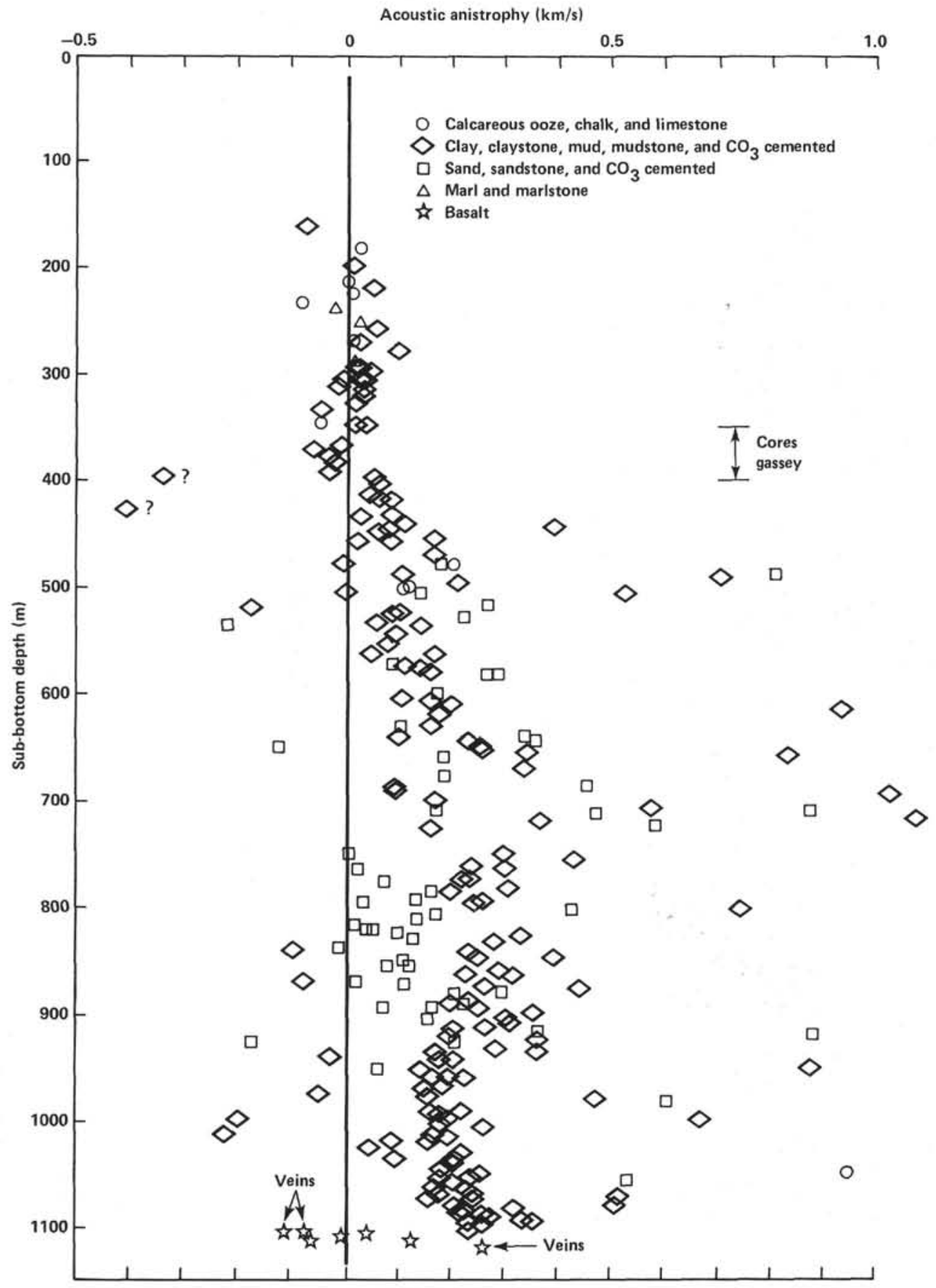

Figure 10. Absolute acoustic anisotropy versus depth, Hole 530A. 


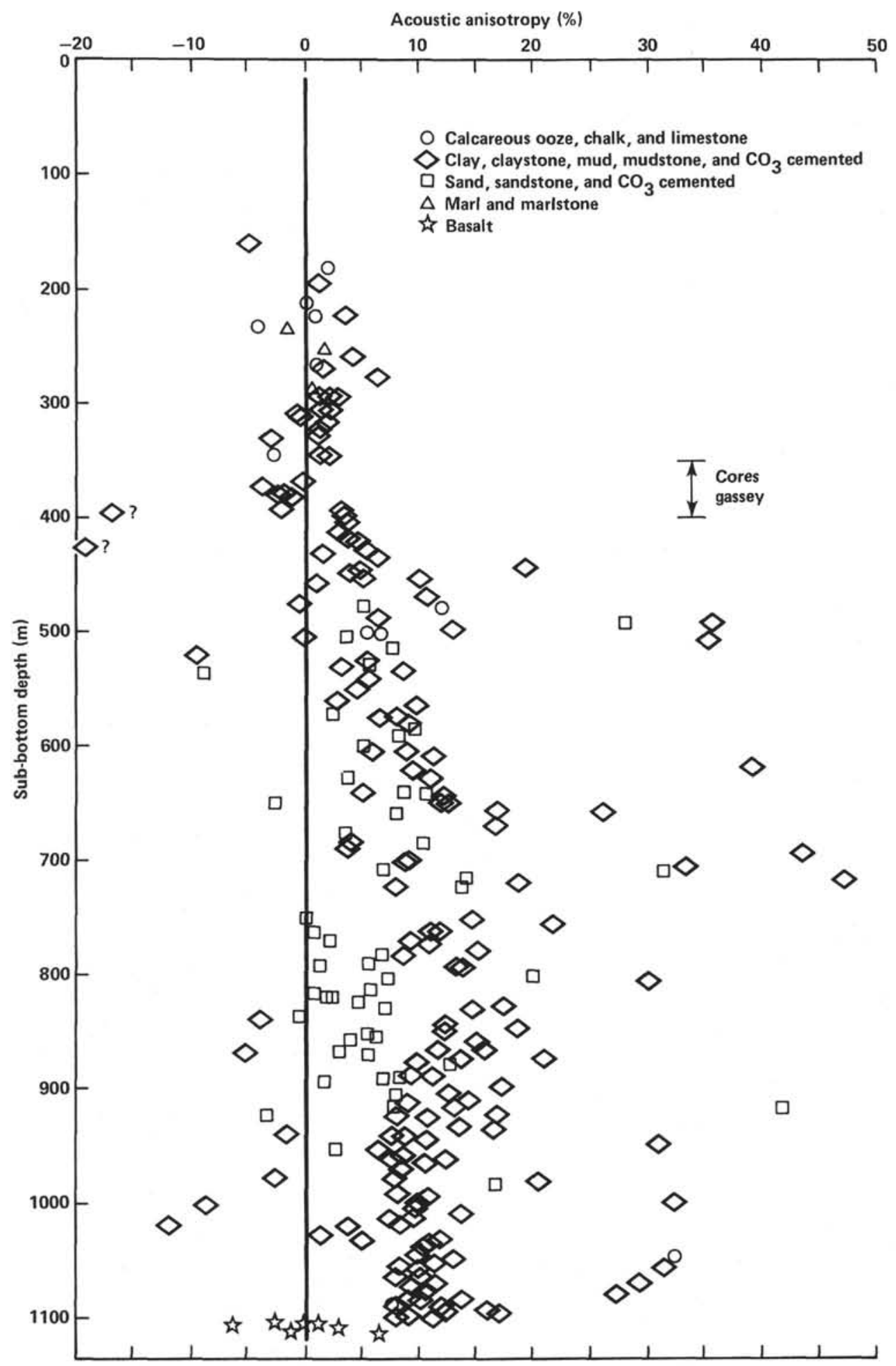

Figure 11. Percentage acoustic anisotropy versus depth, Hole 530A. 


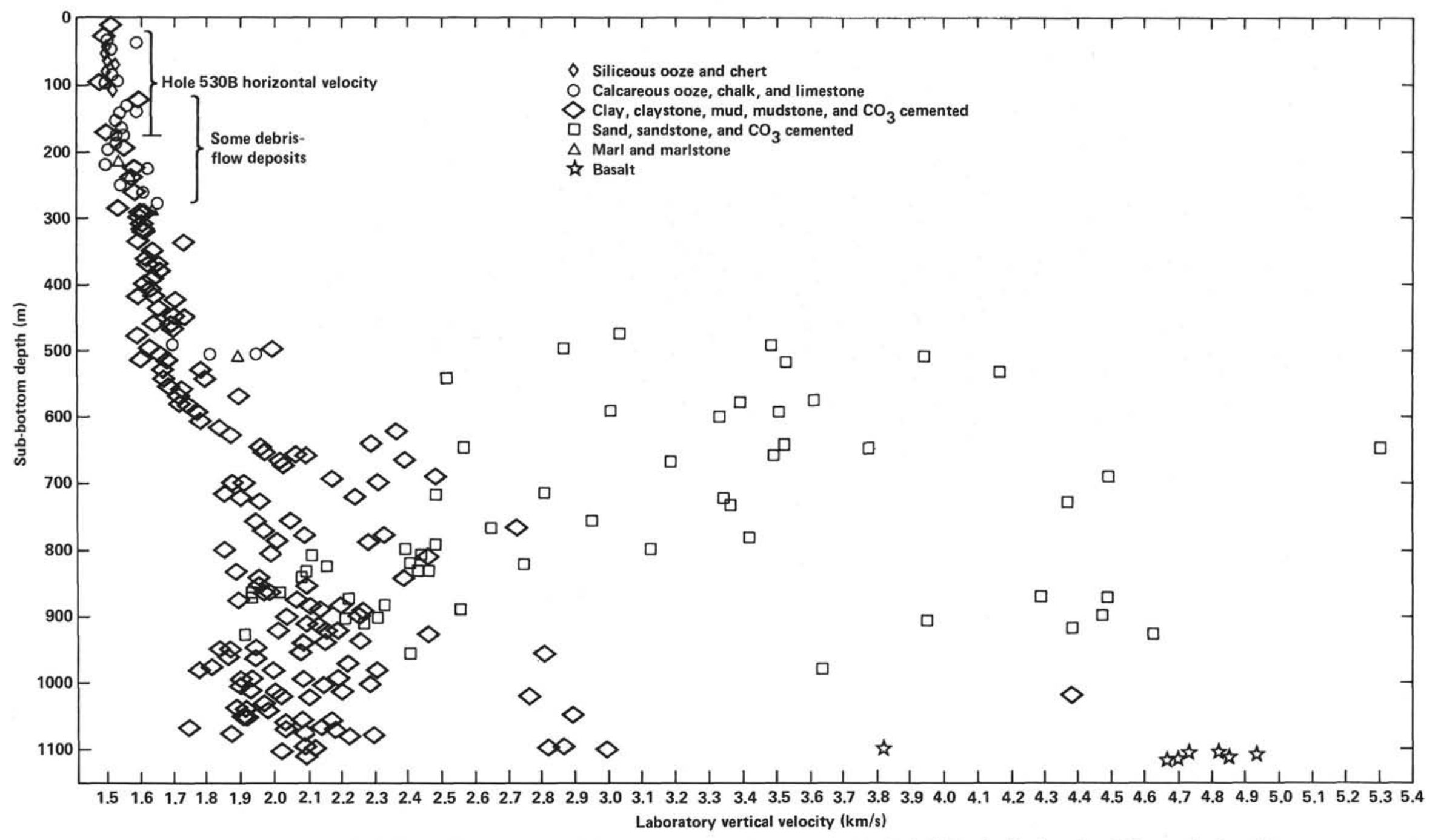

Figure 12. Laboratory vertical velocity versus depth, Hole 530A. (These are at laboratory pressure and temperature. The Hole $530 \mathrm{~B}$ velocities from 0 to $200 \mathrm{~m}$ are horizontal.) 


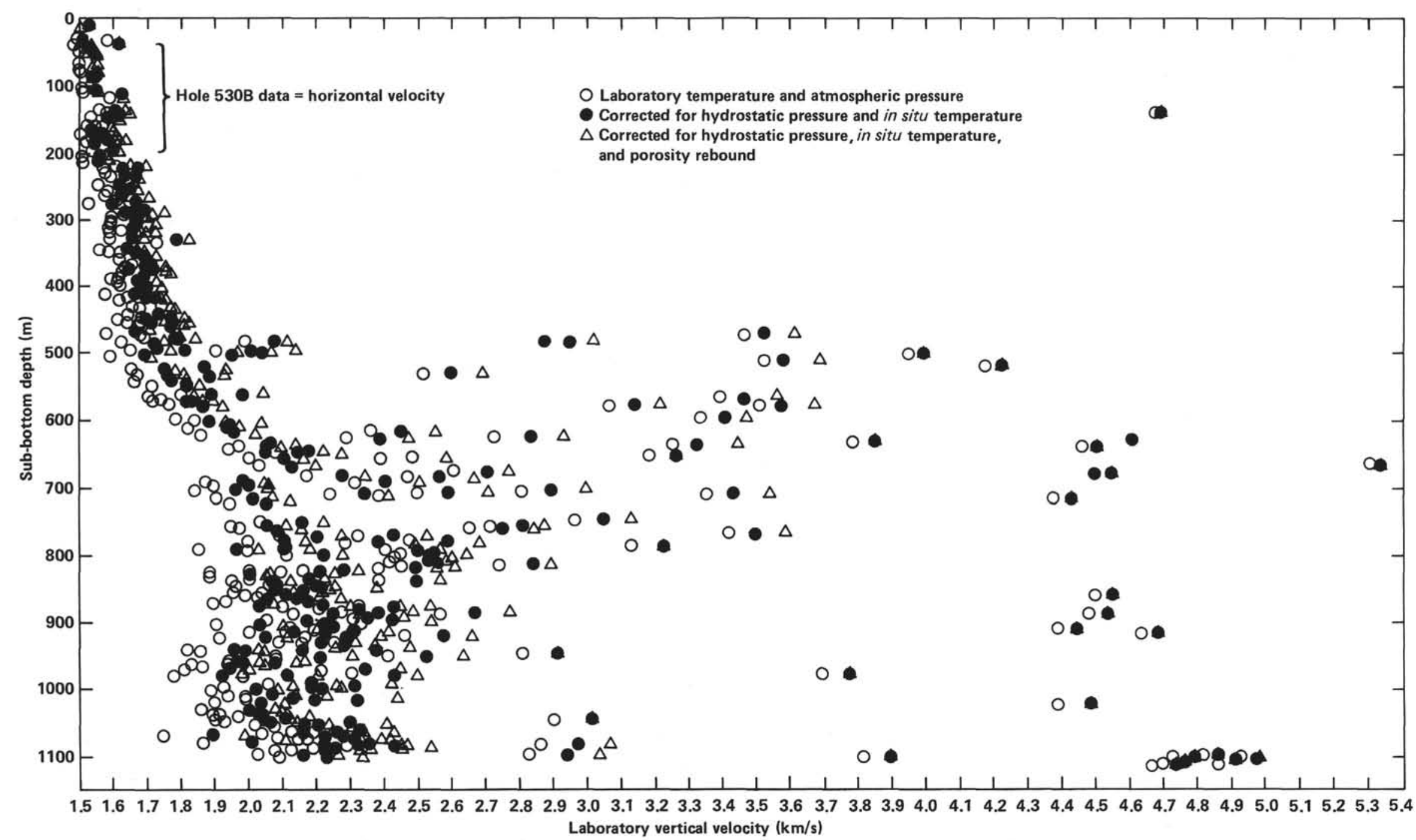

Figure 13. Laboratory vertical velocity versus depth, Hole 530A. The Hole 530B velocities from 0 to $200 \mathrm{~m}$ are horizontal. (Shown are velocities at laboratory conditions, corrected for hydrostatic pressure and temperature, and corrected for hydrostatic pressure, in situ temperature, and porosity rebound.) 


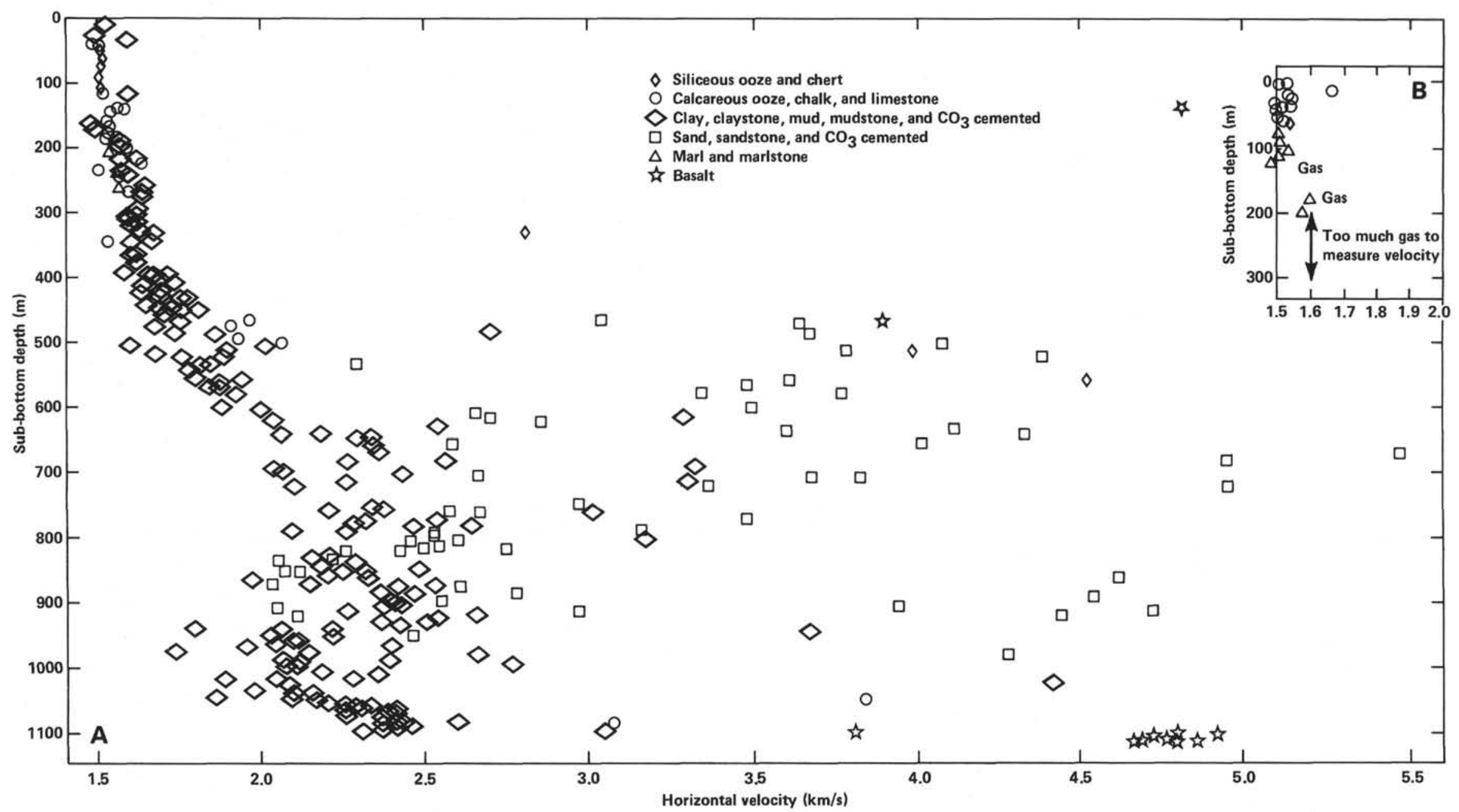

Figure 14. A. Laboratory horizontal velocity versus depth at Site 530 at laboratory pressure and temperature. B. Horizontal laboratory velocity versus depth at Site 532 at laboratory pressure and temperature. 


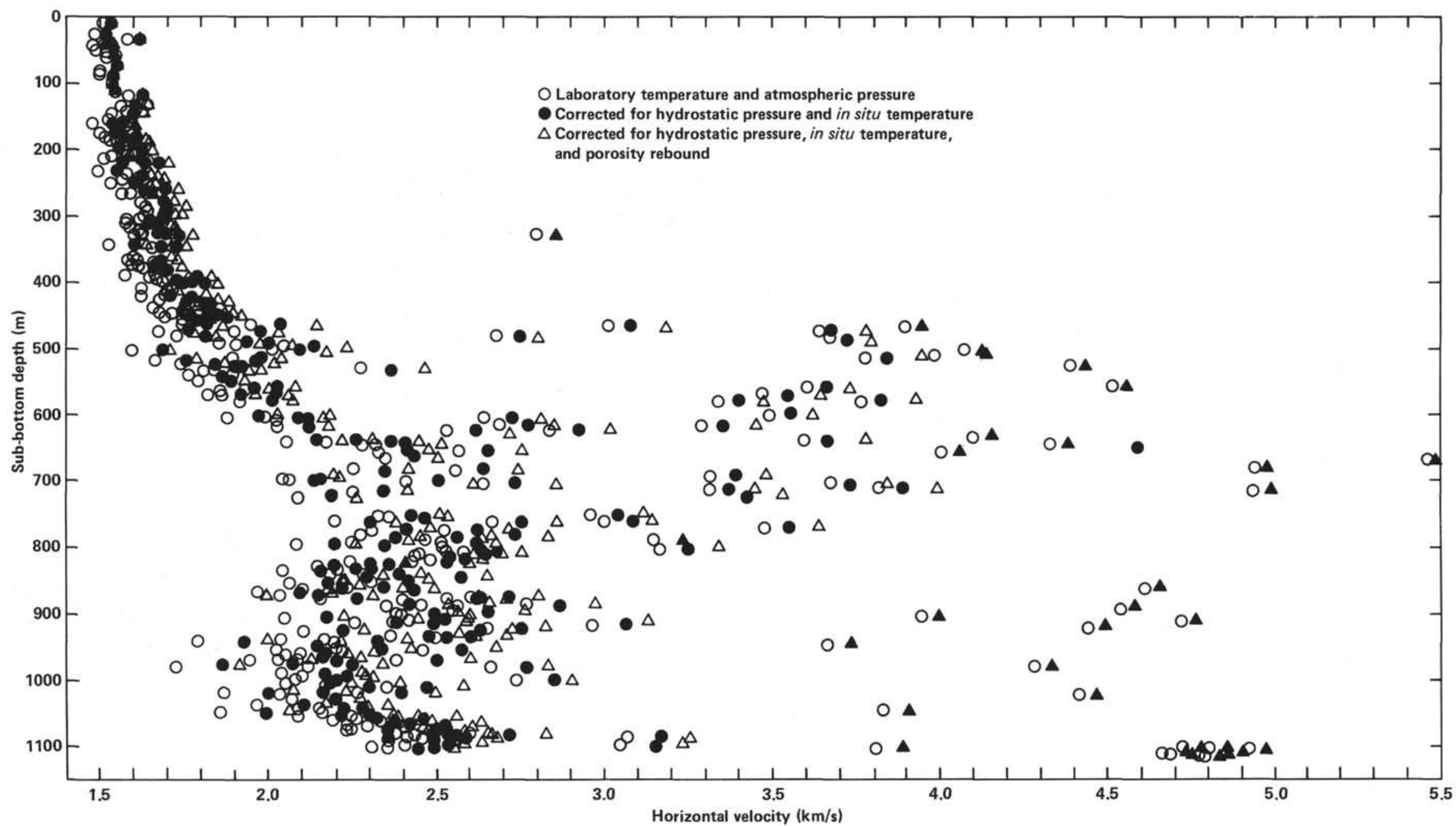

Figure 15. Laboratory horizontal velocity versus depth at Site 530. (These are at laboratory pressure and temperature, corrected for hydrostatic pressure and temperature, and corrected for hydrostatic pressure, in situ temperature, and porosity rebound.) 


\section{R. E. BOYCE}

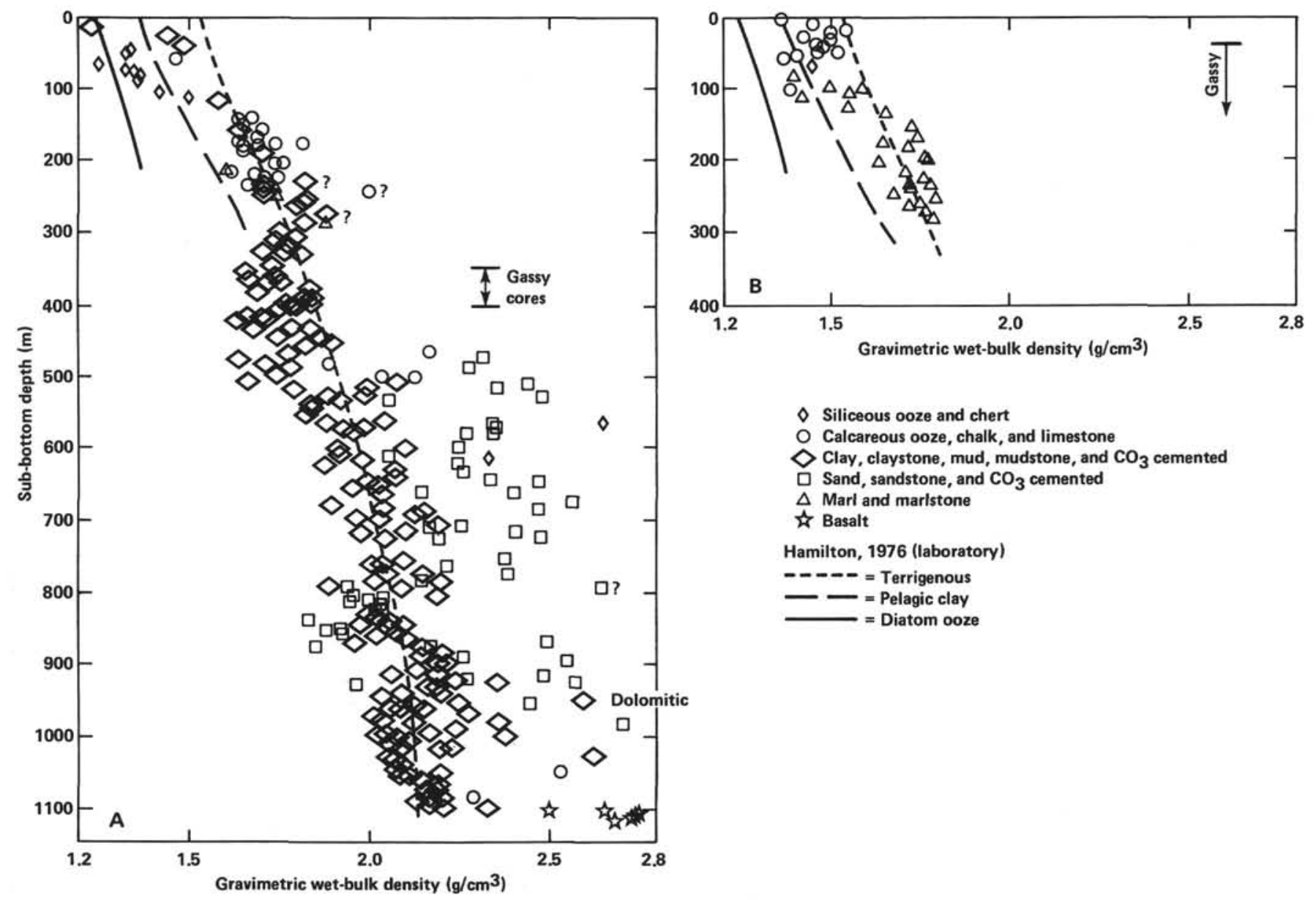

Figure 16. A. Laboratory gravimetric wet-bulk density versus depth, Site 530. B. Site 532. 


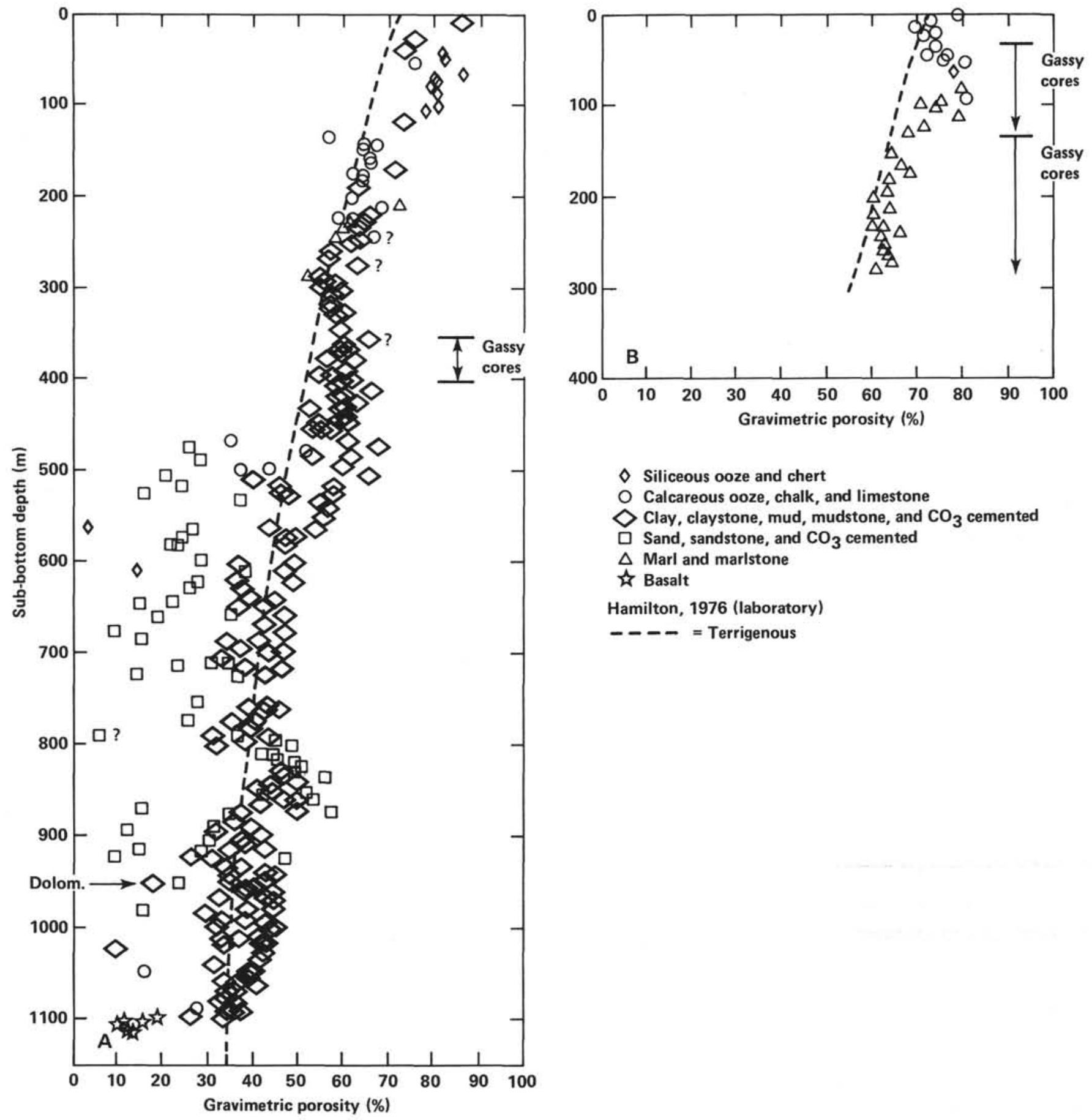

Figure 17. A. Laboratory gravimetric porosity versus depth, Site 530. B. Site 532. 


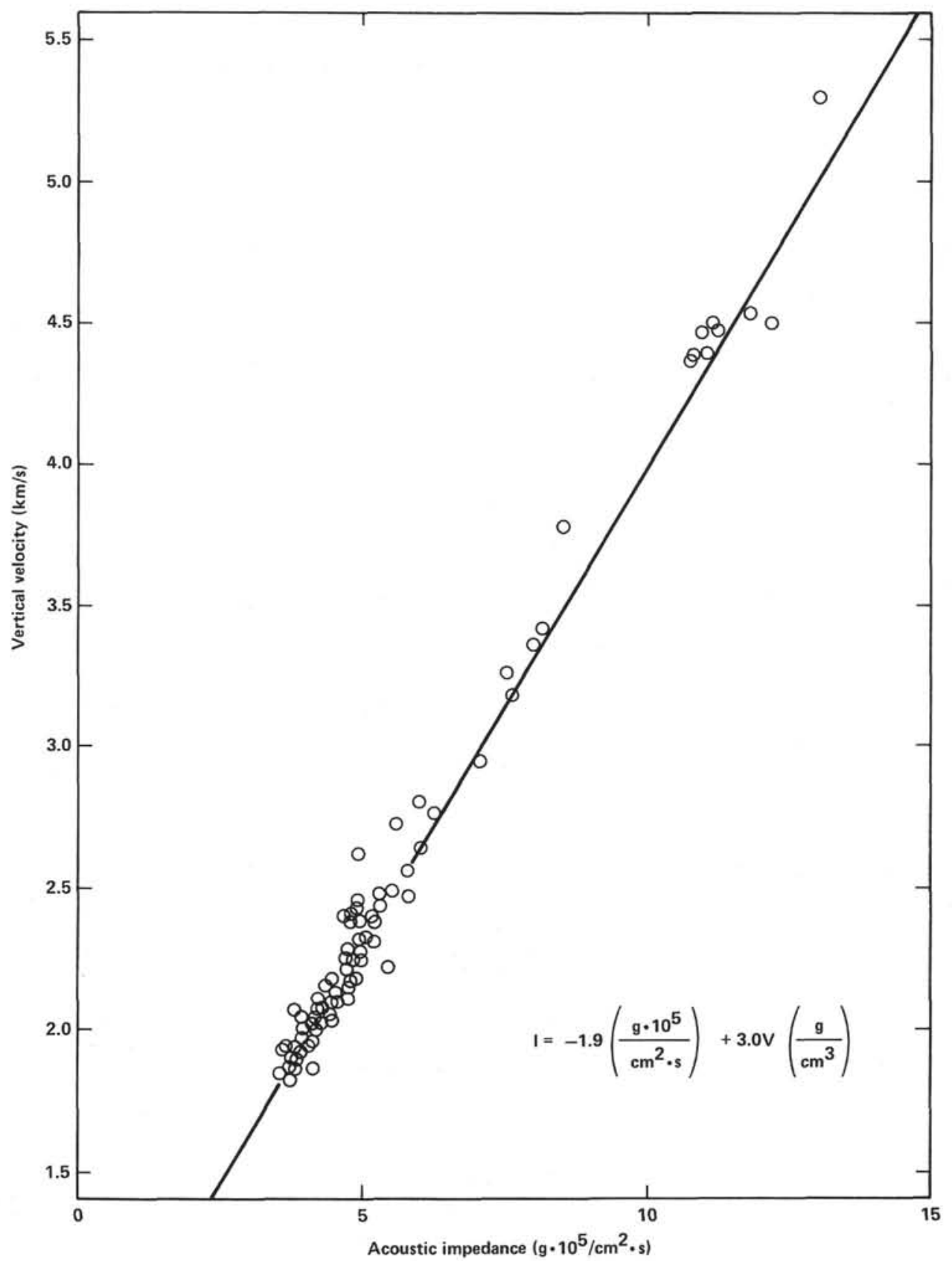

Figure 18. Laboratory vertical velocity versus laboratory impedance from 625 to $945 \mathrm{~m}$, Hole 530A. 


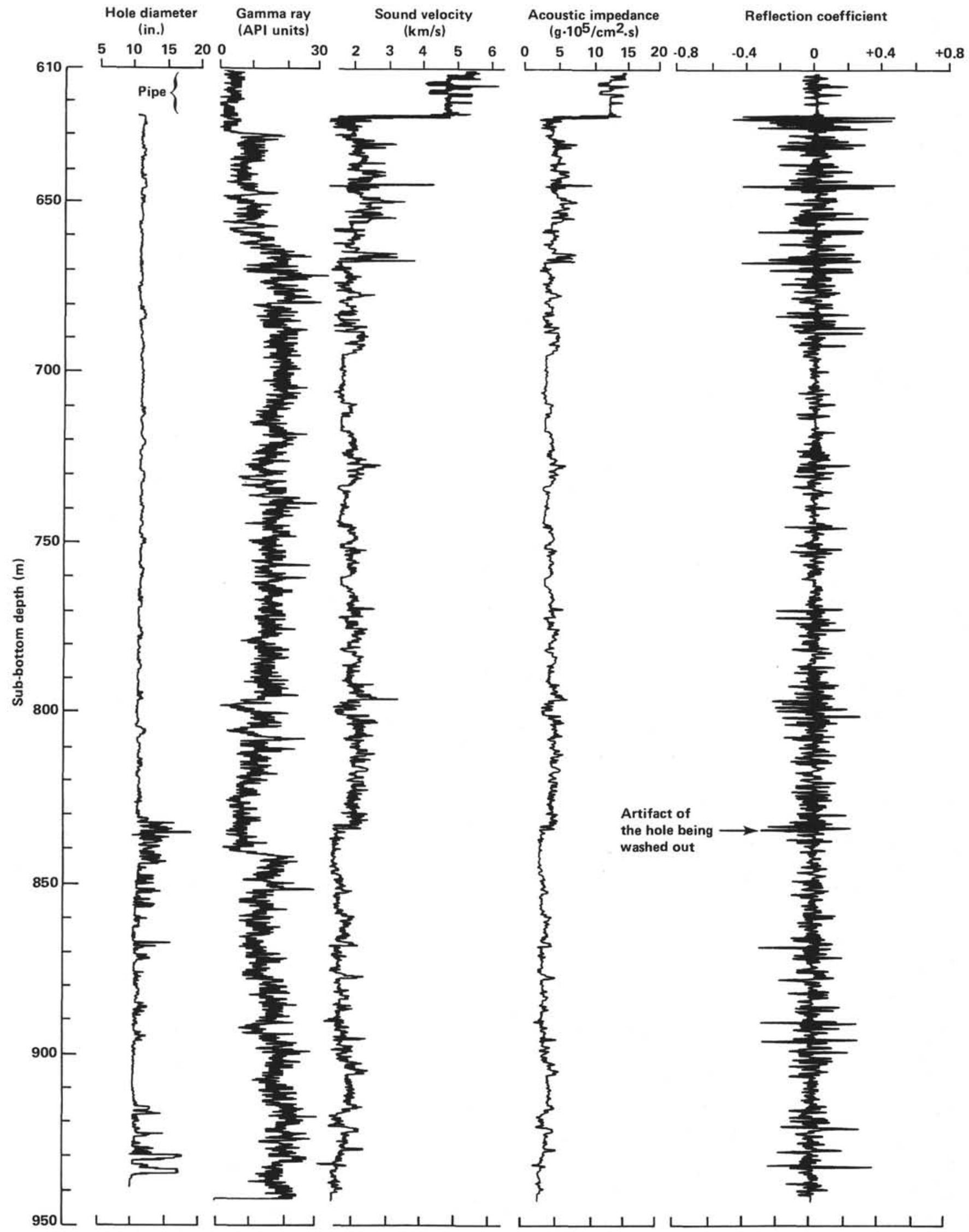

Figure 19. Sonic Log, Sonic Log derived impedance, and Sonic Log-derived reflection coefficients versus depth, Hole 530A. (Vertical depth scale expanded.) 


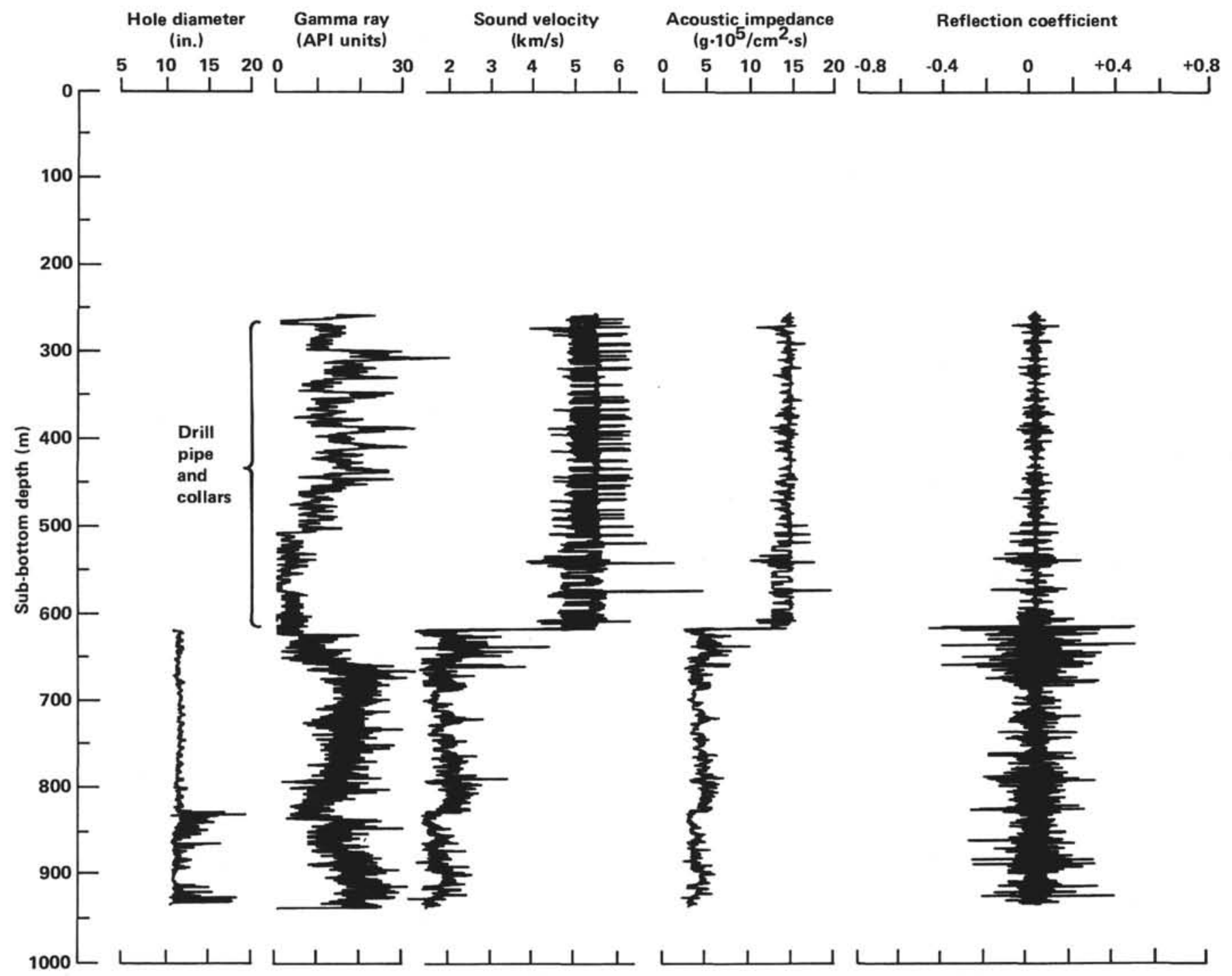

Figure 20. Sonic Log, Sonic Log derived impedance, and Sonic-Log derived reflection coefficients versus depth, Hole 530A. (Vertical depth scale condensed.) 
INDUCTION LOG DATA
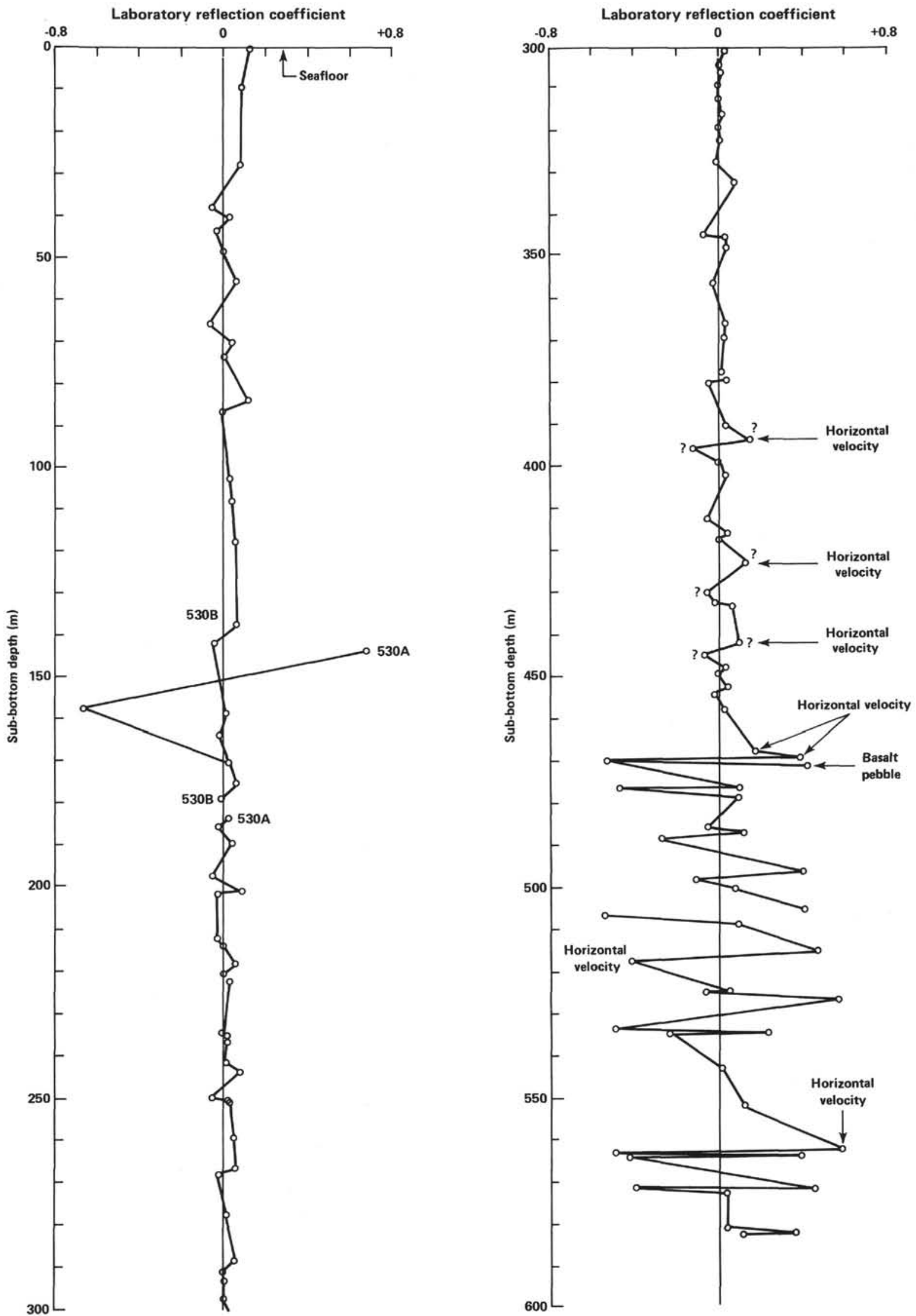

Figure 21. Laboratory derived reflection coefficients versus depth, Site 530. (Vertical depth scale expanded.) 

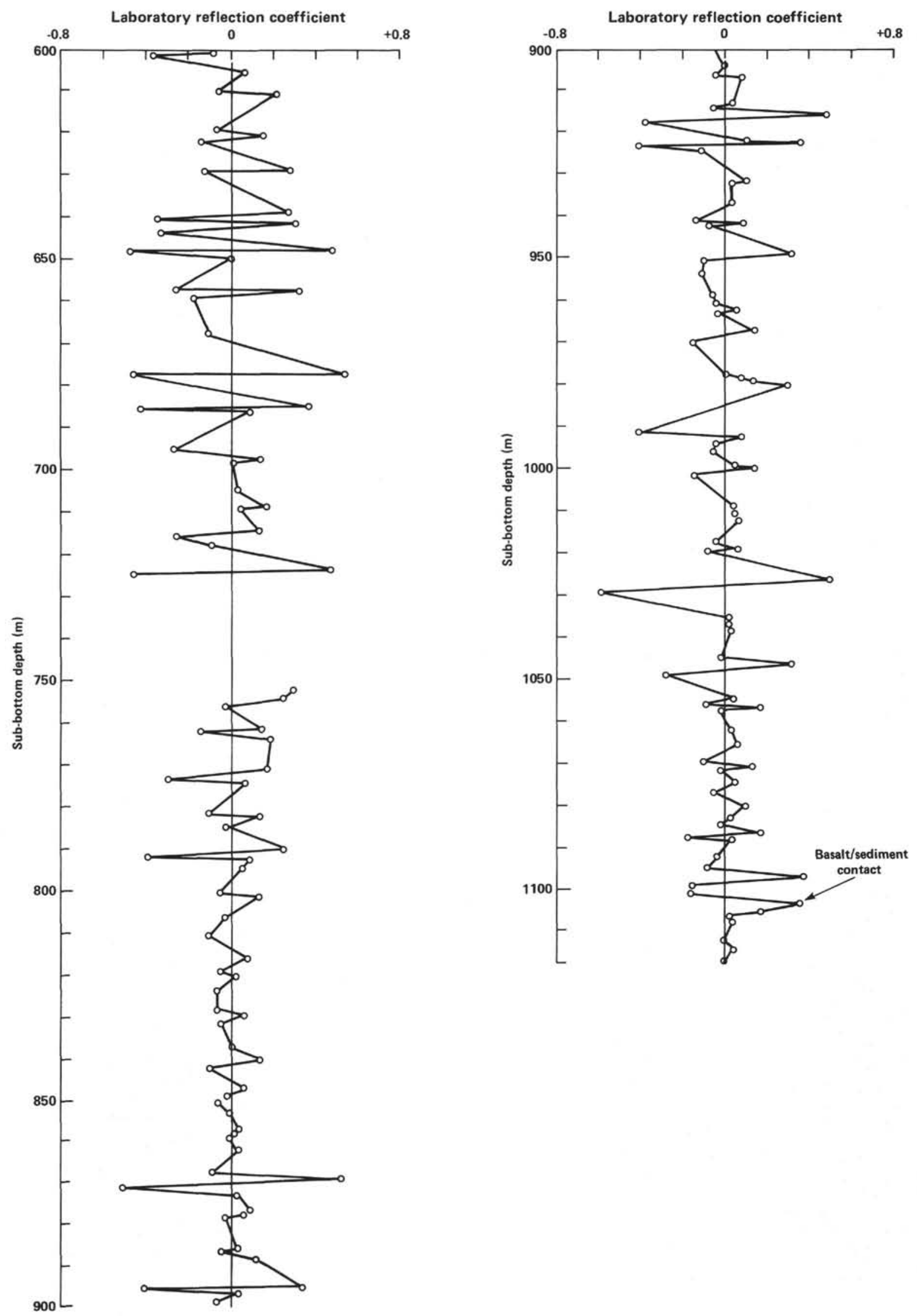

Figure 21. (Continued). 


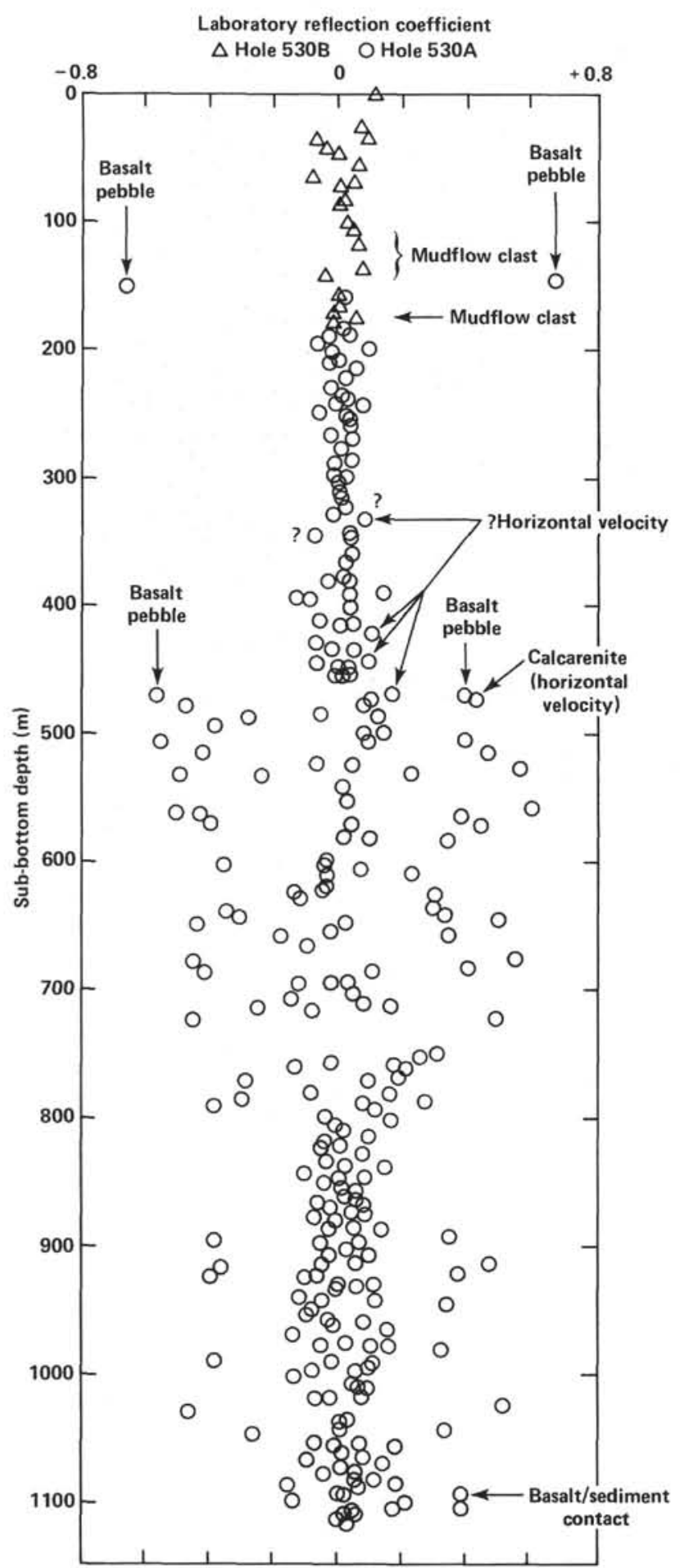

Figure 22. Laboratory-derived reflection coefficients versus depth, Site 530. (Vertical depth scale condensed.)

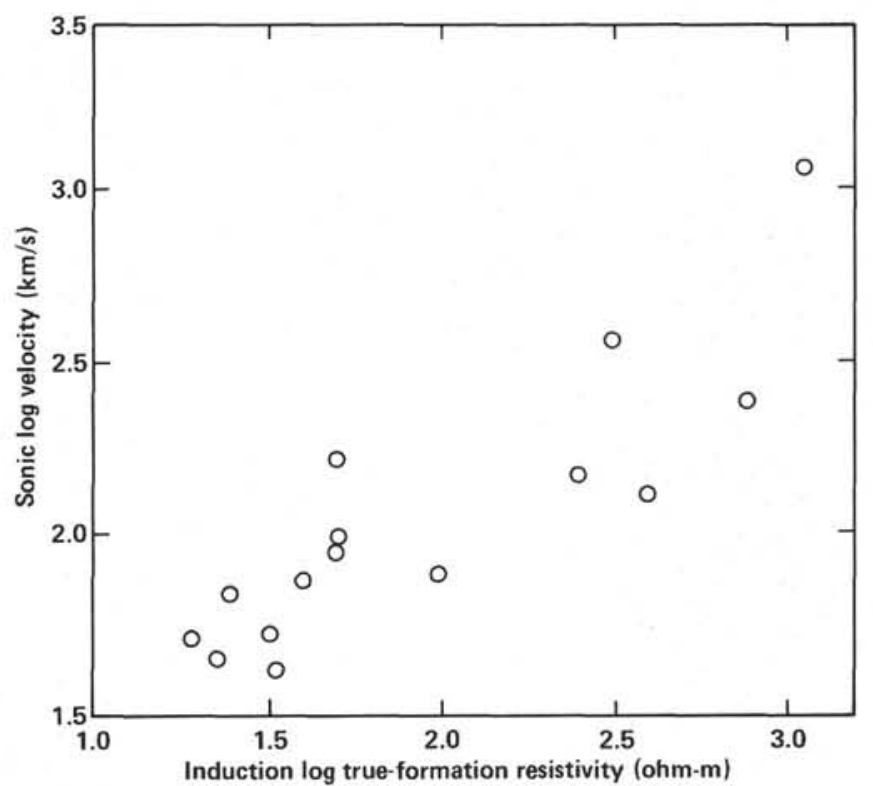

Figure 23. Induction Log true-formation resistivity versus Sonic Log velocity, Hole 530A, 620 to $880 \mathrm{~m}$ (data from Table 3).

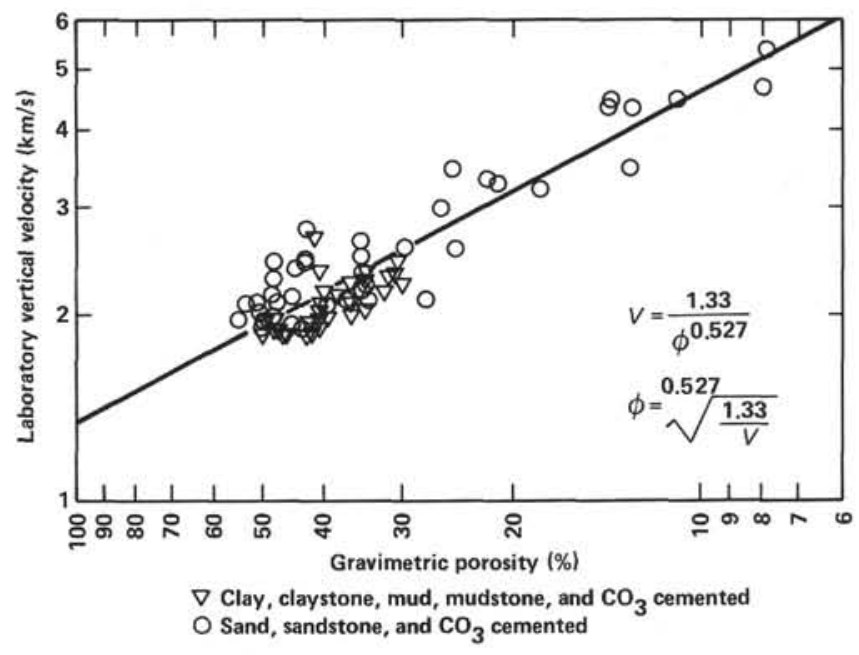

Figure 24. Laboratory vertical velocity versus laboratory gravimetric porosity from 625 to $945 \mathrm{~m}$, Hole 530A. 


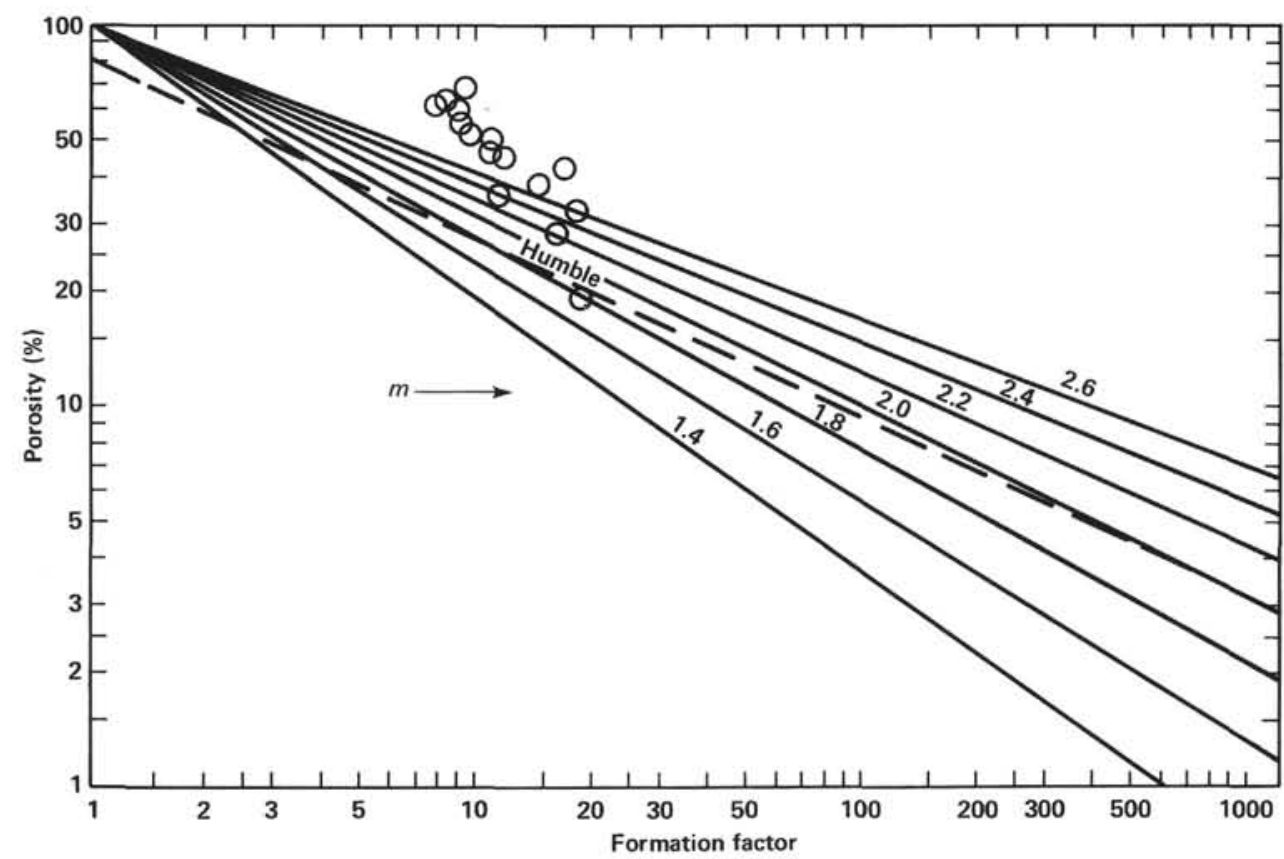

Figure 25. Induction Log derived formation factor versus porosity. Porosity is derived from the velocity $\log$ using Equation 3 derived in Figure 24. The dashed line is the Humble equation (Winsauer et al., 1952) and the solid lines are Archie's (1942) equation for different values of $\mathrm{m}$. Note that the $\mathrm{m}$ values appear to be too high [greater than 2.6)], which could be a result of the velocity from the Sonic Log's being biased too low, particularly in the high porosity formations. If the velocity is too low, then the derived porosity is too large for the high resistivity of the formation, which would cause artificially high $m$ values. However, these do not seriously disagree with the scatter of data in similar plots (Boyce, 1981). 


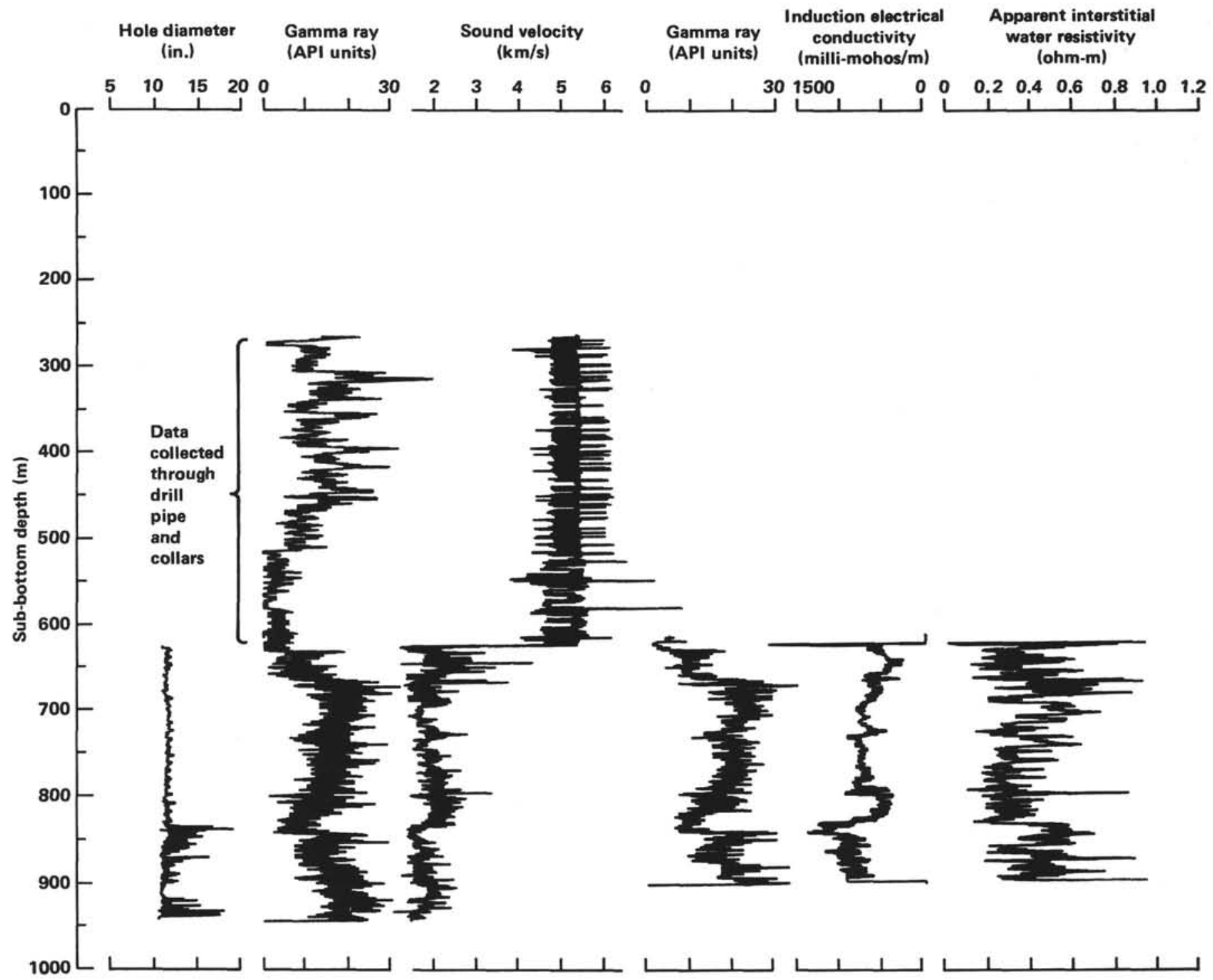

Figure 26. Sonic Log Caliper, Sonic Log Gamma Ray, Sonic Velocity, Induction Log Gamma Ray, Induction Log Electrical Conductivity, and apparent interstitial water resistivity versus depth, Hole 530A.

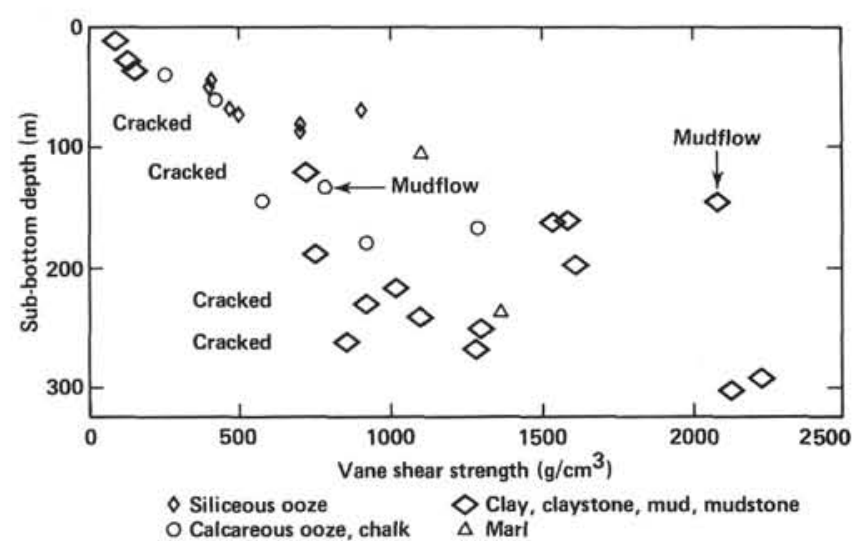

Figure 27. Vane shear strength versus depth, Site 530.

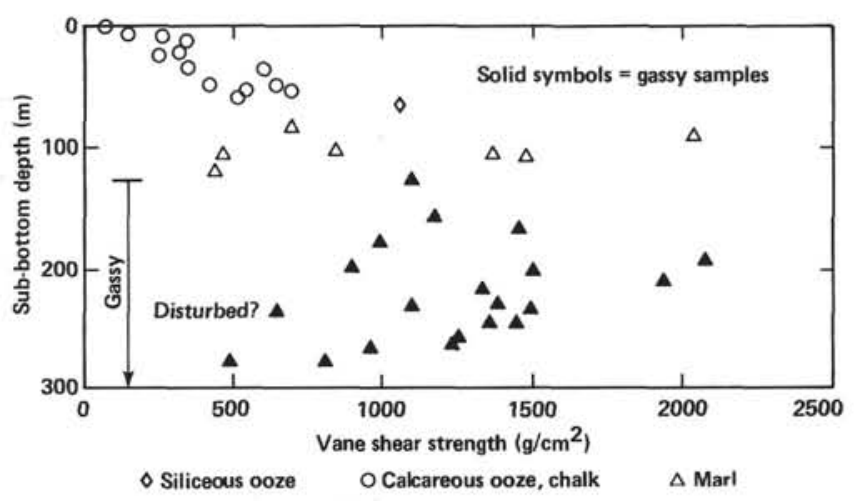

Figure 28. Vane shear strength versus depth, Site 532. 


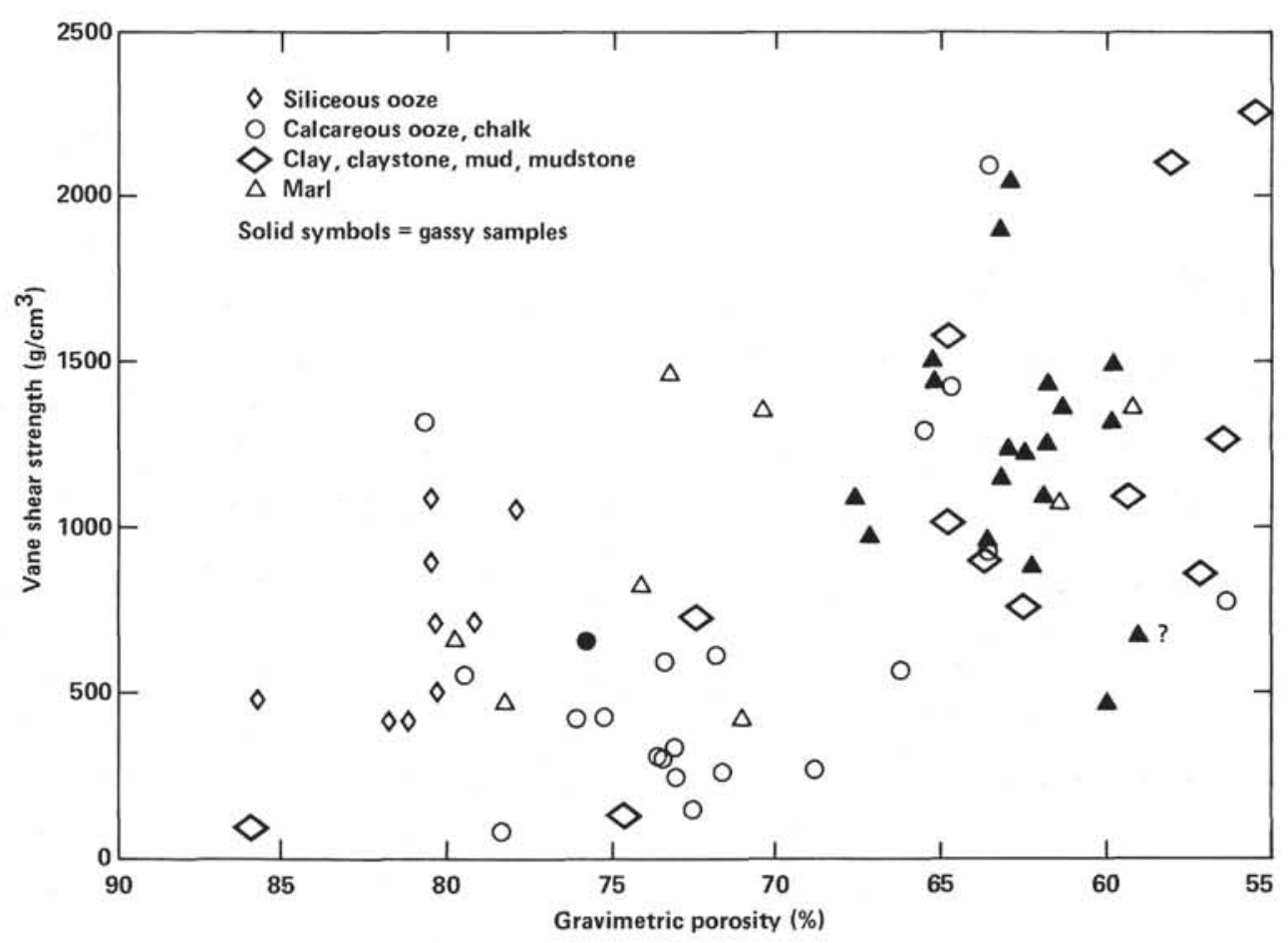

Figure 29. Shear strength versus gravimetric porosity, Sites 530 and 532.

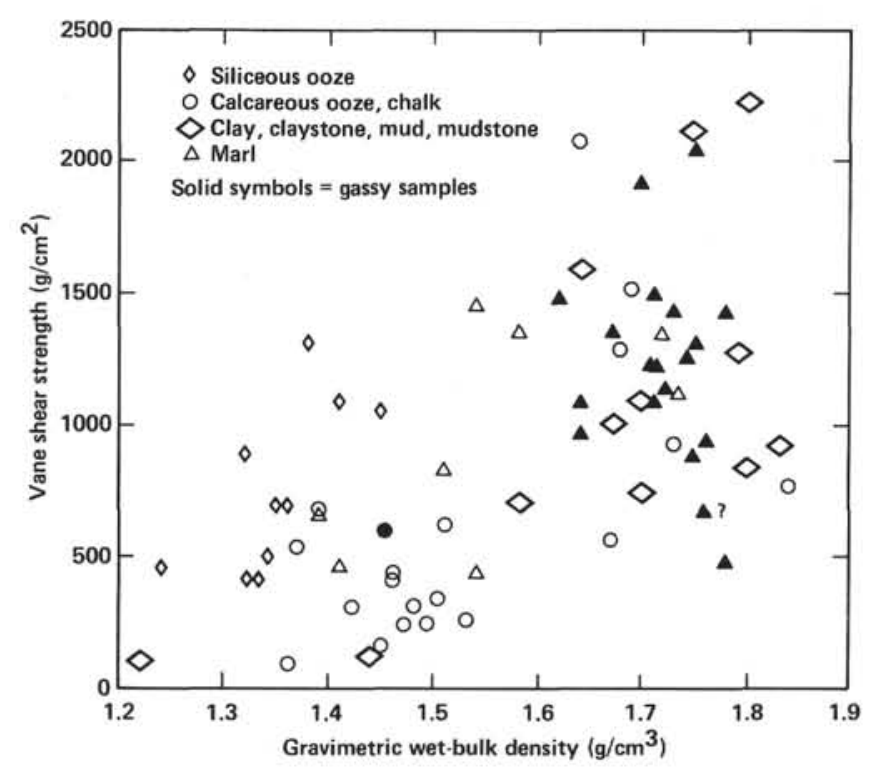

Figure 30. Shear strength versus gravimetric wet-bulk density, Sites 530 and 532 . 


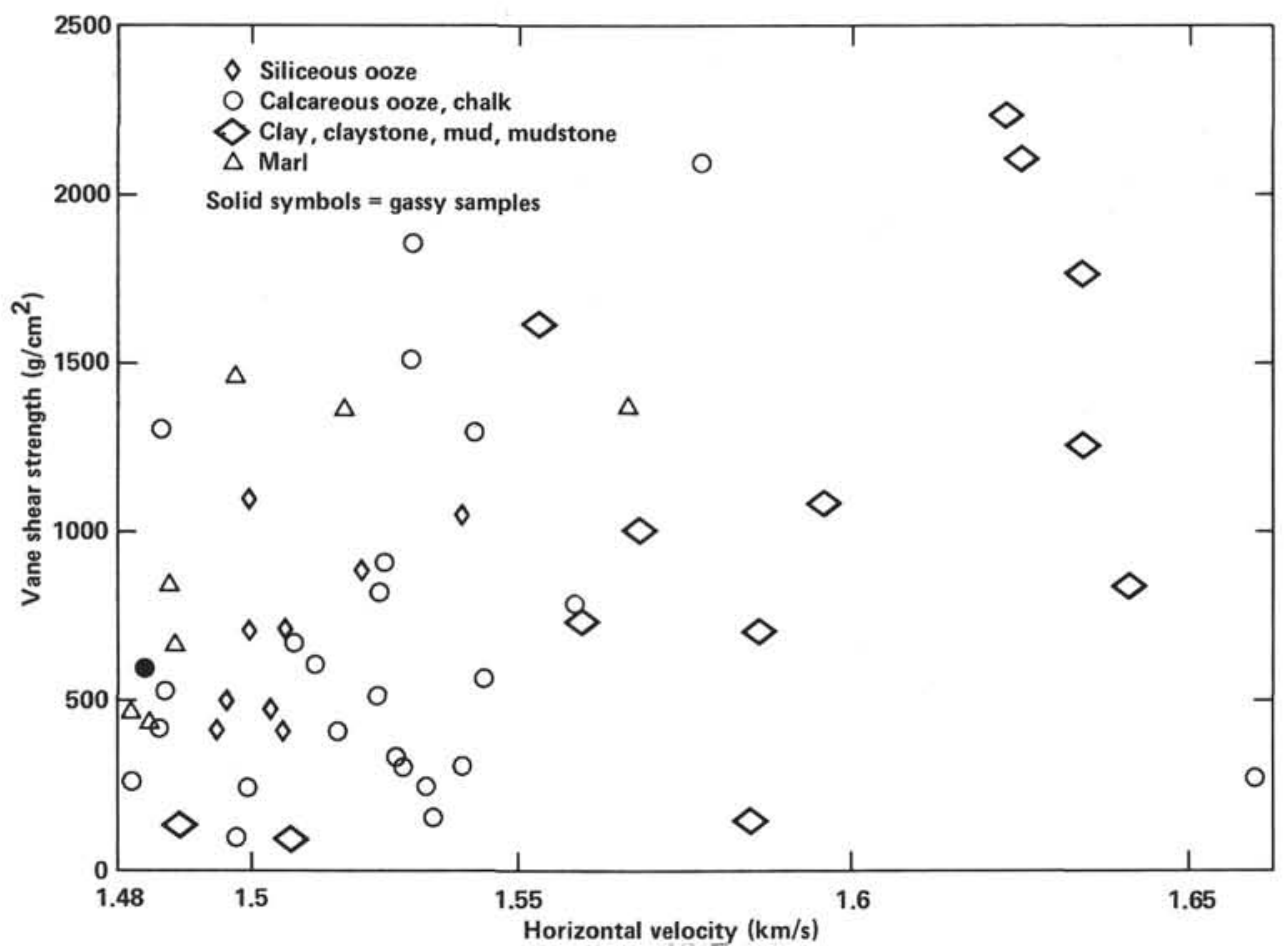

Figure 31. Shear strength versus laboratory velocity, Sites 530 and 532. 